\title{
The semi-parametric Bernstein-von Mises theorem for regression models with symmetric errors
}

\author{
Minwoo Chae ${ }^{1}$, Yongdai Kim² and Bas Kleijn ${ }^{3}$ \\ 1 Department of Mathematics, University of Texas at Austin \\ 2 Department of Statistics, Seoul National University \\ 3 Korteweg-de Vries Institute for Mathematics, University of Amsterdam
}

September 3, 2018

\begin{abstract}
In a smooth semi-parametric model, the marginal posterior distribution for a finite dimensional parameter of interest is expected to be asymptotically equivalent to the sampling distribution of any efficient point-estimator. The assertion leads to asymptotic equivalence of credible and confidence sets for the parameter of interest and is known as the semi-parametric Bernstein-von Mises theorem. In recent years, it has received much attention and has been applied in many examples. We consider models in which errors with symmetric densities play a role; more specifically, it is shown that the marginal posterior distributions of regression coefficients in the linear regression and linear mixed effect models satisfy the semi-parametric Bernstein-von Mises assertion. As a consequence, Bayes estimators in these models achieve frequentist inferential optimality, as expressed e.g. through Hájek's convolution and asymptotic minimax theorems. Conditions for the prior on the space of error densities are relatively mild and well-known constructions like the Dirichlet process mixture of normal densities and random series priors constitute valid choices. Particularly, the result provides an efficient estimate of regression coefficients in the linear mixed effect model, for which no other efficient point-estimator was known previously.
\end{abstract}

\section{Introduction}

In this paper, we give an asymptotic, Bayesian analysis of models with errors that are distributed symmetrically. The observations $\mathbf{X}=\left(X_{1}, \ldots, X_{n}\right)^{T} \in \mathbb{R}^{n}$ are modeled by,

$$
\mathrm{X}=\boldsymbol{\mu}+\boldsymbol{\epsilon}
$$

where $\boldsymbol{\mu}=\left(\mu_{1}, \ldots, \mu_{n}\right)^{T}$ and $\boldsymbol{\epsilon}=\left(\epsilon_{1}, \ldots, \epsilon_{n}\right)^{T}$. Here the mean vector $\boldsymbol{\mu}$ is non-random and parametrized by a finite dimensional parameter $\theta$, and the distribution of the error vector $\boldsymbol{\epsilon}$ is symmetric in the sense that $\boldsymbol{\epsilon}$ has the same distribution as $-\boldsymbol{\epsilon}$. Since the error has a symmetric but otherwise unknown distribution, the model is semi-parametric. Examples of models of the form (1.1) are the symmetric location model (where $\mu_{i}=\theta \in \mathbb{R}$,), and 
the linear regression model (where $\mu_{i}=\theta^{T} Z_{i}$ for given covariates $Z_{i} \in \mathbb{R}^{p}$ ). Moreover, the form (1.1) includes models with dependent errors, like linear mixed effect models.

The main goal of this paper is to prove the semi-parametric Bernstein-von Mises (BvM) assertion for models of the form (1.1) with symmetric error distributions. Roughly speaking we show that the marginal posterior distribution of the parameter of interest $\theta$ is asymptotically normal, centered on an efficient estimator with variance equal to the inverse Fisher information matrix. As a result, statistical inference based on the posterior distribution satisfies frequentist criteria of optimality.

Various sets of sufficient conditions for the semi-parametric BvM theorem based on the full LAN (local asymptotic normality) expansion (i.e. the LAN expansion with respect to both the finite and infinite dimensional parameters [25]) have been developed in [3, 7, 29]. The full LAN expansion, however, is conceptually inaccessible and technically difficult to verify. Because the models we consider are adaptive [4], we can consider a simpler type of LAN expansion that involves only the parameter of interest, albeit that the expansion must be valid under data distributions that differ slightly from the one on which the expansion is centred. We call this property misspecified $L A N$ and prove that it holds for the models of the form (1.1) and that, together with other regularity conditions, it implies the semi-parametric BvM assertion.

While the BvM theorem for parametric Bayesian models is well established (e.g. [21, 23]), the semi-parametric BvM theorem is still being studied very actively: initial examples [9, 11] of simple semi-parametric problems with simple choices for the prior demonstrated failures of marginals posteriors to display BvM-type asymptotic behaviour. Subsequently, positive semi-parametric BvM results have been established in these and various other examples, including models in survival analysis ([18, 19]), multivariate normal regression models with growing numbers of parameters $([5,12,17])$ and discrete probability measures ([6]). More delicate notions like finite sample properties and second-order asymptotics are considered in [26, 30, 38].

Regarding models of the form (1.1), there is a sizable amount of literature on efficient point-estimation in the symmetric location problem ([2, 27, 31]) and linear regression models ([4]). By contrast, to date no efficient point-estimator for the regression coefficients in the linear mixed effect model has been found; the semi-parametric BvM theorem proved below, however, implies that the Bayes estimator is efficient! To the authors' best knowledge, this paper provides the first efficient semi-parametric estimator in the linear mixed effect model. A numerical study given in section 5 supports the view that the Bayes estimator is superior to previous methods of estimation.

This paper is organized as follows: section 2 proves the semi-parametric BvM assertion for all smooth adaptive models (c.f. the misspecified LAN expansion). In sections 3 and 4 we study the linear regression model and linear mixed effect model, respectively. For each, we consider two common choices for the nuisance prior, a Dirichlet process mixture and a series prior, and we show that both lead to validity of the BvM assertion. Results of 
numerical studies are presented in section 5 .

\section{Notation and conventions}

For two real values $a$ and $b, a \wedge b$ and $a \vee b$ are the minimum and maximum of $a$ and $b$, respectively, and $a_{n} \lesssim b_{n}$ signifies that $a_{n}$ is smaller than $b_{n}$ up to a constant multiple independent of $n$. Lebesgue measures are denoted by $\mu ;|\cdot|$ represents the Euclidean norm on $\mathbb{R}^{d}$. The capitals $P_{\eta}, P_{\theta, \eta}$ etc. denote the probability measures associated with densities that we write in lower case, $p_{\eta}, p_{\theta, \eta}$ etc. (where it is always clear from the context which dominating measure $\mu$ is involved). The corresponding log densities are indicated with $\ell_{\eta}, \ell_{\theta, \eta}$ etc. Hellinger and total-variational metrics are defined as $h^{2}\left(p_{1}, p_{2}\right)=\int\left(\sqrt{p_{1}}-\right.$

$\left.\sqrt{p_{2}}\right)^{2} d \mu$ and $d_{V}\left(p_{1}, p_{2}\right)=\int\left|p_{1}-p_{2}\right| d \mu$, respectively. The expectation of a random variable $X$ under a probability measure $P$ is denoted by $P X$. The notation $P_{0}$ always represents the true probability which generates the observation and $X^{o}=X-P_{0} X$ is the centered version of a random variable $X$. The indicator function for a set $A$ is denoted $1_{A}$. For a class of measurable functions $\mathcal{F}$, the quantities $N(\epsilon, \mathcal{F}, d)$ and $N_{[]}(\epsilon, \mathcal{F}, d)$ represent the $\epsilon$-covering and -bracketing numbers [33] with respect to a (semi)metric $d$.

\section{Misspecified LAN and the semi-parametric BvM theorem}

In this section, we prove the semi-parametric BvM theorem for smooth adaptive models, i.e. those that satisfy the misspecified LAN expansion defined below.

\subsection{Misspecified local asymptotic normality}

Consider a sequence of statistical models $\mathscr{P}^{(n)}=\left\{P_{\theta, \eta}^{(n)}: \theta \in \Theta, \eta \in \mathcal{H}\right\}$ on measurable spaces $\left(\mathscr{X}^{(n)}, \mathscr{A}^{(n)}\right)$, parametrized by a finite dimensional parameter $\theta$ of interest and an infinite dimensional nuisance parameter $\eta$. Assume that $\Theta$ is a subset of $\mathbb{R}^{p}, \mathcal{H}$ is a metric space equipped with the associated Borel $\sigma$-algebra and $P_{\theta, \eta}^{(n)}$ has density $x \mapsto p_{\theta, \eta}^{(n)}(x)$ with respect to some $\sigma$-finite measures $\mu^{(n)}$ dominating $\mathscr{P}^{(n)}$.

Let $X^{(n)}$ be a $\mathscr{X}^{(n)}$-valued random element following $P_{0}^{(n)}$ and assume that $P_{0}^{(n)}=$ $P_{\theta_{0}, \eta_{0}}^{(n)}$ for some $\theta_{0} \in \Theta$ and $\eta_{0} \in \mathcal{H}$. We say that a sequence of statistical models $\mathscr{P}^{(n)}$ satisfies the misspecified $L A N$ expansion if there exists a sequence of vector-valued (componentwise) $L_{2}\left(P_{0}^{(n)}\right)$-functions $\left(g_{n, \eta}\right)$, a sequence $\left(\mathcal{H}_{n}\right)$ of measurable subsets of $\mathcal{H}$ and a sequence $\left(V_{n, \eta}\right)$ of $p \times p$-matrices such that,

$$
\sup _{h \in K} \sup _{\eta \in \mathcal{H}_{n}}\left|\log \frac{p_{\theta_{n}(h), \eta}^{(n)}}{p_{\theta_{0}, \eta}^{(n)}}\left(X^{(n)}\right)-\frac{h^{T}}{\sqrt{n}} g_{n, \eta}\left(X^{(n)}\right)+\frac{1}{2} h^{T} V_{n, \eta} h\right|=o_{P_{0}}(1),
$$

for every compact $K \subset \mathbb{R}^{p}$, where $\theta_{n}(h)$ equals $\theta_{0}+h / \sqrt{n}$. When we know $\eta_{0}$, property 2.1. is nothing but the usual parametric LAN expansion, where we set $\mathcal{H}_{n}=\left\{\eta_{0}\right\}$. We 
refer to (2.1) as the misspecified LAN expansion because the base for the expansion is $\left(\theta_{0}, \eta\right)$ while rest-terms go to zero under $P_{0}$, which corresponds to the point $\left(\theta_{0}, \eta_{0}\right)$.

Note that the misspecified LAN expansion is simpler than the full LAN expansion used in [3, 7, 29]. Although the misspecified LAN expansion (2.1) can be applied only to the adaptive cases, the verification of (2.1) is not easy due to misspecification and the required uniformity of convergence. LAN expansions have been shown to be valid even under misspecification: in [21] for example, smoothness in misspecified parametric models is expressed through a version of local asymptotic normality under the true distribution of the data, with a likelihood expansion around points in the model where the KullbackLeibler (KL)-divergence with respect to $P_{0}$ is minimal. In models with symmetric error, the point of minimal KL-divergence equals exactly $\theta_{0}$, provided that the misspecified $\eta$ is close enough to $\eta_{0}$ in the sense of $\mathcal{H}_{n}$. This allows the usual LAN expansion at $\theta_{0}$ for fixed $\eta$, that is, the left-hand side of (2.1) is expected to be of order $o_{P_{0}}(1)$. By choosing localizations $\mathcal{H}_{n}$ appropriately, the family of score functions $\left\{\dot{\ell}_{\theta, \eta}: \eta \in \mathcal{H}_{n}\right\}$ is shown to be a Donsker class, which validates $(2.1)$ in models with symmetric errors, where $\dot{\ell}_{\theta, \eta}(x)=\partial \ell_{\theta, \eta}(x) / \partial \theta, g_{n, \eta}\left(X^{(n)}\right)=\sum_{i=1}^{n} \dot{\ell}_{\theta_{0}, \eta}\left(X_{i}\right)$ and $V_{n, \eta}=n^{-1} P_{0}^{(n)}\left[g_{n, \eta} g_{n, \eta_{0}}^{T}\right]$. The score function is not necessarily the pointwise derivative of the log-likelihood, but in most examples (including the models considered in this paper), $g_{n, \eta}=\dot{\ell}_{\theta_{0}, \eta}^{(n)}$ where $\dot{\ell}_{\theta, \eta}^{(n)}=\ell_{\theta, \eta}^{(n)} / \partial \theta$. From now on, since it conveys the natural meaning of derivative, we use the notation $\dot{\ell}_{\theta_{0}, \eta}^{(n)}$ instead of $g_{n, \eta}$.

\subsection{The semi-parametric Bernstein-von Mises theorem}

We use a product prior $\Pi=\Pi_{\Theta} \times \Pi_{\mathcal{H}}$ on the Borel $\sigma$-algebra of $\Theta \times \mathcal{H}$ and denote the posterior distribution by $\Pi\left(\cdot \mid X^{(n)}\right)$. Note that the misspecified LAN property gives rise to an expansion of the log-likelihood that applies only locally in sets $\Theta_{n} \times \mathcal{H}_{n}$, where $\Theta_{n}=\left\{\theta_{0}+h / \sqrt{n}: h \in K\right\}$ (for some compact $K \in \mathbb{R}^{p}$ and appropriate $\mathcal{H}_{n} \subset \mathcal{H}$ ). So for the semi-parametric BvM theorem, the score function $\dot{\ell}_{\theta_{0}, \eta}^{(n)}$ as well as $V_{n, \eta}$ must 'behave nicely' on $\Theta_{n} \times \mathcal{H}_{n}$ and the posterior distribution must concentrate inside $\Theta_{n} \times \mathcal{H}_{n}$. Technically, these requirements are expressed by the following two conditions. For a matrix $A \in \mathbb{R}^{n_{1} \times n_{2}},\|A\|$ represents the operator norm of $A$, defined as $\sup _{x \neq 0}|A x| /|x|$, and if $A$ is a square matrix, $\rho_{\min }(A)$ and $\rho_{\max }(A)$ denote the minimum and maximum eigenvalues of $A$, respectively.

Condition A. (Equicontinuity and non-singularity)

$$
\begin{aligned}
\sup _{\eta \in \mathcal{H}_{n}}\left|\dot{\ell}_{\theta_{0}, \eta}^{(n)}\left(X^{(n)}\right)-\dot{\ell}_{\theta_{0}, \eta_{0}}^{(n)}\left(X^{(n)}\right)\right| & =o_{P_{0}}\left(n^{1 / 2}\right), \\
\sup _{\eta \in \mathcal{H}_{n}}\left\|V_{n, \eta}-V_{n, \eta_{0}}\right\| & =o(1), \\
0<\liminf _{n \rightarrow \infty} \rho_{\min }\left(V_{n, \eta_{0}}\right) \leq \limsup _{n \rightarrow \infty} \rho_{\max }\left(V_{n, \eta_{0}}\right) & <\infty
\end{aligned}
$$


Condition B. (Posterior localization)

$$
\begin{aligned}
P_{0}^{(n)} \Pi\left(\mathcal{H}_{n} \mid X^{(n)}\right) & \rightarrow 1, \\
P_{0}^{(n)} \Pi\left(\sqrt{n}\left|\theta-\theta_{0}\right|>M_{n} \mid X^{(n)}\right) & \rightarrow 0, \text { for every } M_{n} \uparrow \infty .
\end{aligned}
$$

Conditions like (2.2) and (2.3) are to be expected in the context of semi-parametric estimation (see, e.g., Theorem 25.54 of [34]). Condition (2.2) amounts to asymptotic equicontinuity and is implied whenever scores form a Donsker class, a well-known sufficient condition in semi-parametric efficiency (see [34). Condition (2.3) is implied whenever the $L_{2}\left(P_{0}^{(n)}\right)$-norm of the difference between scores at $\left(\theta_{0}, \eta\right)$ and $\left(\theta_{0}, \eta_{0}\right)$ vanishes as $\eta$ converges to $\eta_{0}$ in Hellinger distance, c.f. (3.12); it controls variations of the information matrix as $\eta$ converges to $\eta_{0}$ with $\mathcal{H}_{n}$. Condition (2.4) guarantees that the Fisher information matrix does not develop singularities as the sample size goes to infinity.

Condition 2.5 formulates a requirement of posterior consistency in the usual sense, and sufficient conditions are well-known [1, 20, 28, 36. Condition (2.6) requires $n^{-1 / 2}$-rate of convergence rate for the marginal posterior distribution for the parameter of interest. Though some authors remark that 2.6) appears to be rather too strong [38], clearly, 2.6] is a necessary condition (since it follows directly from the BvM assertion). The proof of condition 2.6 is demanding in a technical sense and forms the most difficult part of this analysis and most others [3].

We say the prior $\Pi_{\Theta}$ is thick at $\theta_{0}$ if it has a strictly positive and continuous Lebesgue density in a neighborhood of $\theta_{0}$. The following theorem states the BvM theorem for semi-parametric models that are smooth in the sense of the misspecified LAN expansion.

Theorem 2.1. Consider statistical models $\left\{P_{\theta, \eta}^{(n)}: \theta \in \Theta, \eta \in \mathcal{H}\right\}$ with a product prior $\Pi=\Pi_{\Theta} \times \Pi_{\mathcal{H}}$. Assume that $\Pi_{\Theta}$ is thick at $\theta_{0}$ and that 2.1) as well as Conditions $A$ and $B$ hold. Then,

$$
\sup _{B}\left|\Pi\left(\sqrt{n}\left(\theta-\theta_{0}\right) \in B \mid X^{(n)}\right)-N_{\Delta_{n}, V_{n, \eta_{0}}^{-1}}(B)\right| \rightarrow 0,
$$

in $P_{0}^{(n)}$-probability, where,

$$
\Delta_{n}=\frac{1}{\sqrt{n}} V_{n, \eta_{0}}^{-1} \dot{\ell}_{\theta_{0}, \eta_{0}}^{(n)}\left(X^{(n)}\right) .
$$

Proof. Note first that 2.5) implies that $\Pi_{\mathcal{H}}\left(\mathcal{H}_{n}\right)>0$ for large enough $n$. Let $\Pi_{\mathcal{H}_{n}}$ be the probability measure obtained by restricting $\Pi_{\mathcal{H}}$ to $\mathcal{H}_{n}$ and next re-normalizing, and $\Pi_{\mathcal{H}_{n}}\left(\cdot \mid X^{(n)}\right)$ be the corresponding posterior distribution. Then, for any measurable set $B$ in $\Theta$,

$$
\begin{aligned}
\Pi(\theta & \left.\in B \mid X^{(n)}\right)=\Pi\left(\theta \in B, \eta \in \mathcal{H}_{n} \mid X^{(n)}\right)+\Pi\left(\theta \in B, \eta \in \mathcal{H}_{n}^{c} \mid X^{(n)}\right) \\
& =\Pi_{\mathcal{H}_{n}}\left(\theta \in B \mid X^{(n)}\right) \Pi\left(\eta \in \mathcal{H}_{n} \mid X^{(n)}\right)+\Pi\left(\theta \in B, \eta \in \mathcal{H}_{n}^{c} \mid X^{(n)}\right),
\end{aligned}
$$

so we have,

$$
\sup _{B}\left|\Pi\left(\theta \in B \mid X^{(n)}\right)-\Pi_{\mathcal{H}_{n}}\left(\theta \in B \mid X^{(n)}\right)\right| \rightarrow 0
$$


in $P_{0}^{(n)}$-probability. Therefore it is sufficient to prove the BvM assertion with the priors $\Pi_{\mathcal{H}_{n}}$.

Particularly,

$$
\Pi_{\mathcal{H}_{n}}\left(\sqrt{n}\left|\theta-\theta_{0}\right|>M_{n} \mid X^{(n)}\right)=\frac{\Pi\left(\sqrt{n}\left|\theta-\theta_{0}\right|>M_{n}, \eta \in \mathcal{H}_{n} \mid X^{(n)}\right)}{\Pi\left(\eta \in \mathcal{H}_{n} \mid X^{(n)}\right)},
$$

converges to 0 in $P_{0}^{(n)}$-probability by 2.5 and (2.6). Using (2.1), 2.2 and (2.3), we obtain,

$$
\sup _{h \in K} \sup _{\eta \in \mathcal{H}_{n}}\left|\log \frac{p_{\theta_{n}(h), \eta}^{(n)}}{p_{\theta_{0}, \eta}^{(n)}}\left(X^{(n)}\right)-\frac{h^{T}}{\sqrt{n}} \dot{\ell}_{\theta_{0}, \eta_{0}}^{(n)}\left(X^{(n)}\right)+\frac{1}{2} h^{T} V_{n, \eta_{0}} h\right|=o_{P_{0}}(1),
$$

for every compact $K \subset \mathbb{R}^{p}$. Let,

$$
b_{1}(h)=\inf _{\eta \in \mathcal{H}_{n}} \frac{p_{\theta_{n}(h), \eta}^{(n)}\left(X^{(n)}\right)}{p_{\theta_{0}, \eta}^{(n)}\left(X^{(n)}\right)}, \quad \text { and } \quad b_{2}(h)=\sup _{\eta \in \mathcal{H}_{n}} \frac{p_{\theta_{n}(h), \eta}^{(n)}\left(X^{(n)}\right)}{p_{\theta_{0}, \eta}^{(n)}\left(X^{(n)}\right)} .
$$

Then, trivially, we have,

$$
b_{1}(h) \leq \frac{\int p_{\theta_{n}(h), \eta}^{(n)}\left(X^{(n)}\right) d \Pi_{\mathcal{H}_{n}}(\eta)}{\int p_{\theta_{0}, \eta}^{(n)}\left(X^{(n)}\right) d \Pi_{\mathcal{H}_{n}}(\eta)} \leq b_{2}(h),
$$

and the quantity,

$$
\sup _{h \in K}\left|b_{k}(h)-\frac{h^{T}}{\sqrt{n}} \dot{\ell}_{\theta_{0}, \eta_{0}}^{(n)}\left(X^{(n)}\right)+\frac{1}{2} h^{T} V_{n, \eta_{0}} h\right|,
$$

is bounded above by the left-hand side of 2.9 for $k=1,2$. As a result,

$$
\sup _{h \in K}\left|\log \frac{\int p_{\theta_{n}(h), \eta}^{(n)}\left(X^{(n)}\right) d \Pi_{\mathcal{H}_{n}}(\eta)}{\int p_{\theta_{0}, \eta}^{(n)}\left(X^{(n)}\right) d \Pi_{\mathcal{H}_{n}}(\eta)}-\frac{h^{T}}{\sqrt{n}} \dot{\ell}_{\theta_{0}, \eta_{0}}^{(n)}\left(X^{(n)}\right)+\frac{1}{2} h^{T} V_{n, \eta_{0}} h\right|=o_{P_{0}}(1),
$$

because $\left|c_{2}\right| \leq\left|c_{1}\right| \vee\left|c_{3}\right|$ for all real numbers $c_{1}, c_{2}$ and $c_{3}$ with $c_{1} \leq c_{2} \leq c_{3}$. The remainder of the proof is (almost) identical to the proof for parametric models [21, 23], replacing the parametric likelihood by $\theta \mapsto \int p_{\theta, \eta}^{(n)}\left(X^{(n)}\right) d \Pi_{\mathcal{H}_{n}}(\eta)$ as in [3], details of which can be found in Theorem 3.1.1 of [8].

\section{Semi-parametric BvM for linear regression models}

Let $\mathcal{H}$ be the set of all continuously differentiable densities $\eta$ defined on $\mathbb{D}=(-r, r)$ (for some $r \in(0, \infty])$ such that $\eta(x)>0$ and $\eta(x)=\eta(-x)$ for every $x \in \mathbb{D}$. Equip $\mathcal{H}$ with the Hellinger metric. We consider a model for data satisfying,

$$
X_{i}=\theta^{T} Z_{i}+\epsilon_{i}, \quad \text { for } i=1, \ldots, n,
$$

where $Z_{i}$ 's are $p$-dimensional non-random covariates and the errors $\epsilon_{i}$ are assumed to form an i.i.d. sample from a distribution with density $\eta \in \mathcal{H}$. We prove the BvM theorem for the regression coefficient $\theta$. 
Let $P_{\theta, \eta, i}$ denote the probability measure with density $x \mapsto \eta\left(x-\theta^{T} Z_{i}\right)$ and $\dot{\ell}_{\theta, \eta, i}=$ $\partial \ell_{\theta, \eta, i} / \partial \theta$. Also let $P_{\eta}$ be the probability measure with density $p_{\eta}=\eta$ and $s_{\eta}(x)=$ $-\partial \ell_{\eta}(x) / \partial x$. Let $P_{\theta, \eta}^{(n)}$ represent the product measure $P_{\theta, \eta, 1} \times \cdots \times P_{\theta, \eta, n}$ and let $\dot{\ell}_{\theta, \eta}^{(n)}=$ $\sum_{i=1}^{n} \dot{\ell}_{\theta, \eta, i}$. With slight abuse of notation, we treat $p_{\theta, \eta, i}, \ell_{\theta, \eta, i}$ and $\dot{\ell}_{\theta, \eta, i}$ as either functions of $x$ or the corresponding random variables when they are evaluated at $x=X_{i}$. For example, $\dot{\ell}_{\theta, \eta, i}$ represents either the function $x \mapsto \dot{\ell}_{\theta, \eta, i}(x): \mathbb{D} \mapsto \mathbb{R}^{p}$ or the random vector $\dot{\ell}_{\theta, \eta, i}\left(X_{i}\right)$. We treat $p_{\theta, \eta}^{(n)}, \ell_{\theta, \eta}^{(n)}$ and $\dot{\ell}_{\theta, \eta}^{(n)}$ similarly.

Let $\theta_{0} \in \Theta$ and $\eta_{0} \in \mathcal{H}$ be the true regression coefficient and error density in the model (3.1). Define specialized KL-balls in $\Theta \times \mathcal{H}$ of the form,

$$
B_{n}(\epsilon)=\left\{(\theta, \eta): \sum_{i=1}^{n} K\left(p_{\theta_{0}, \eta_{0}, i}, p_{\theta, \eta, i}\right) \leq n \epsilon^{2}, \sum_{i=1}^{n} V\left(p_{\theta_{0}, \eta_{0}, i}, p_{\theta, \eta, i}\right) \leq C_{2} n \epsilon^{2}\right\},
$$

where $K\left(p_{1}, p_{2}\right)=\int \log \left(p_{1} / p_{2}\right) d P_{1}, V\left(p_{1}, p_{2}\right)=\int\left(\log \left(p_{1} / p_{2}\right)-K\left(p_{1}, p_{2}\right)\right)^{2} d P_{1}$, and $C_{2}$ is some positive constant (see [14]). Define the mean Hellinger distance $h_{n}$ on $\Theta \times \mathcal{H}$ by,

$$
h_{n}^{2}\left(\left(\theta_{1}, \eta_{1}\right),\left(\theta_{2}, \eta_{2}\right)\right)=\frac{1}{n} \sum_{i=1}^{n} h^{2}\left(p_{\theta_{1}, \eta_{1}, i}, p_{\theta_{2}, \eta_{2}, i}\right) .
$$

Let $v_{\eta}=P_{\eta_{0}}\left[s_{\eta} s_{\eta_{0}}\right]$ and,

$$
V_{n, \eta}=\frac{1}{n} P_{0}^{(n)}\left[\dot{\ell}_{\theta_{0}, \eta}^{(n)} \dot{\ell}_{\theta_{0}, \eta_{0}}^{(n) T}\right]
$$

It is easy to see that $V_{n, \eta}=v_{\eta} \mathbf{Z}_{n}$, where $\mathbf{Z}_{n}=n^{-1} \sum_{i=1}^{n} Z_{i} Z_{i}^{T}$.

We say that a sequence of real-valued stochastic processes $\left\{Y_{n}(t): t \in T\right\},(n \geq 1)$, is asymptotically tight if it is asymptotically tight in the space of bounded functions on $T$ with the uniform norm [33]. A vector-valued stochastic process is asymptotic tight if each of its components is asymptotically tight.

Theorem 3.1. Suppose that $\sup _{i \geq 1}\left|Z_{i}\right| \leq L$ for some constant $L>0, \liminf { }_{n} \rho_{\min }\left(\mathbf{Z}_{n}\right)>$ 0 and $v_{\eta_{0}}>0$. The prior for $(\theta, \eta)$ is a product $\Pi=\Pi_{\Theta} \times \Pi_{\mathcal{H}}$, where $\Pi_{\Theta}$ is thick at $\theta_{0}$. Suppose also that there exist an $N \geq 1$, a sequence $\epsilon_{n} \rightarrow 0$ with $n \epsilon_{n}^{2} \rightarrow \infty$, and partitions $\Theta=\Theta_{n, 1} \cup \Theta_{n, 2}$ and $\mathcal{H}=\mathcal{H}_{n, 1} \cup \mathcal{H}_{n, 2}$ such that $\eta_{0} \in \mathcal{H}_{n, 1}$ and

$$
\begin{aligned}
\log N\left(\epsilon_{n} / 36, \Theta_{n, 1} \times \mathcal{H}_{n, 1}, h_{n}\right) & \leq n \epsilon_{n}^{2}, \\
\log \Pi\left(B_{n}\left(\epsilon_{n}\right)\right) & \geq-\frac{1}{4} n \epsilon_{n}^{2}, \\
\log \left(\Pi_{\Theta}\left(\Theta_{n, 2}\right)+\Pi_{\mathcal{H}}\left(\mathcal{H}_{n, 2}\right)\right) & \leq-\frac{5}{2} n \epsilon_{n}^{2},
\end{aligned}
$$

for all $n \geq N$. For some $\bar{M}_{n} \uparrow \infty$, with $\epsilon_{n} \bar{M}_{n} \rightarrow 0$, let $\mathcal{H}_{n}=\left\{\eta \in \mathcal{H}_{n, 1}: h\left(\eta, \eta_{0}\right)<\bar{M}_{n} \epsilon_{n}\right\}$ and assume that there exist a continuous $L_{2}\left(P_{\eta_{0}}\right)$-function $Q$ and an $\epsilon_{0}>0$ such that,

$$
\sup _{|y|<\epsilon_{0}} \sup _{\eta \in \mathcal{H}^{N}}\left|\frac{\ell_{\eta}(x+y)-\ell_{\eta}(x)}{y}\right| \vee\left|\frac{s_{\eta}(x+y)-s_{\eta}(x)}{y}\right| \leq Q(x),
$$

where $\mathcal{H}^{N}=\cup_{n=N}^{\infty} \mathcal{H}_{n}$. Furthermore, assume that the sequence of stochastic processes,

$$
\left\{\frac{1}{\sqrt{n}}\left(\dot{\ell}_{\theta, \eta}^{(n)}-P_{0}^{(n)} \dot{\ell}_{\theta, \eta}^{(n)}\right):\left|\theta-\theta_{0}\right|<\epsilon_{0}, \eta \in \mathcal{H}^{N}\right\},
$$


indexed by $(\theta, \eta)$ is asymptotically tight. Then the assertion of the BvM theorem 2.1 holds for $\theta$.

Since the observations are not i.i.d., we consider the mean Hellinger distance $h_{n}$ as in [14. Conditions (3.5) are required for the convergence rate of $h_{n}\left((\theta, \eta),\left(\theta_{0}, \eta_{0}\right)\right)$ to be $\epsilon_{n}$, which in turn implies that the convergence rates of $\left|\theta-\theta_{0}\right|$ and $h\left(\eta, \eta_{0}\right)$ are $\epsilon_{n}$ (c.f. Lemma 3.1). In fact, we only need to prove (3.5) with arbitrary rate $\epsilon_{n}$ because the so-called no-bias condition $\sup _{\eta \in \mathcal{H}_{n}} P_{0} \dot{\ell}_{\theta_{0}, \eta}^{(n)}=o_{P_{0}}\left(n^{-1 / 2}\right)$ holds trivially by the symmetry, which plays an important role to prove (2.1)-(2.3) as in frequentist literature (see Chapter 25 of [35]). Condition (3.6), which is technical in nature, is easily satisfied. For a random design, (3.7) is asymptotically tight if and only if the class of score functions forms a Donsker class, and sufficient conditions for the latter are well established in empirical process theory. Since observations are not i.i.d. due to the non-randomness of covariates, (3.7) does not converge in distribution to a Gaussian process. Here, asymptotic tightness of (3.7) merely assures that the supremum of its norm is of order $O_{P_{0}}(1)$. Asymptotic tightness holds under a finite bracketing integral condition (where the definition of the bracketing number is extended to non-i.i.d. observations in a natural way). For sufficient conditions for asymptotic tightness with non-i.i.d. observations, readers are referred to section 2.11 of $[33$.

We prove Theorem 3.1 by checking the misspecified LAN condition as well as Conditions A and B, whose proofs are sketched in the three following subsections respectively. Detailed proofs are provided in the appendix.

\subsection{Proof of Misspecified LAN}

Note that $P_{0}^{(n)} \dot{\ell}_{\theta_{0}, \eta}^{(n)}=0$ for every $\eta \in \mathcal{H}$ by the symmetry of $\eta$ and $\eta_{0}$. This enables writing the left-hand side of 2.1) as,

$$
\log \frac{p_{\theta_{n}(h), \eta}^{(n)}}{p_{\theta_{0}, \eta}^{(n)}}\left(X^{(n)}\right)-\frac{h^{T}}{\sqrt{n}} \dot{\ell}_{\theta_{0}, \eta}^{(n)}\left(X^{(n)}\right)+\frac{1}{2} h^{T} V_{n, \eta} h=A_{n}(h, \eta)+B_{n}(h, \eta),
$$

where,

$$
\begin{aligned}
& A_{n}(h, \eta)=\left(\ell_{\theta_{n}(h), \eta}^{(n)}-\ell_{\theta_{0}, \eta}^{(n)}-\frac{h^{T}}{\sqrt{n}} \dot{\ell}_{\theta_{0}, \eta}^{(n)}\right)^{o}, \\
& B_{n}(h, \eta)=P_{0}^{(n)}\left(\ell_{\theta_{n}(h), \eta}^{(n)}-\ell_{\theta_{0}, \eta}^{(n)}\right)+\frac{1}{2} h^{T} V_{n, \eta} h .
\end{aligned}
$$

It suffices to prove that $A_{n}(h, \eta)$ and $B_{n}(h, \eta)$ converge to zero uniformly over $h \in K$ and $\eta \in \mathcal{H}^{N}$, in $P_{0}^{(n)}$-probability, for every compact set $K$.

Note that $A_{n}(h, \eta)$ is equal to,

$$
\frac{h^{T}}{\sqrt{n}} \int_{0}^{1}\left(\dot{\ell}_{\theta_{n}(t h), \eta}^{(n)}-\dot{\ell}_{\theta_{0}, \eta}^{(n)}\right)^{o} d t
$$


by Taylor expansion, so for a compact set $K$, we have,

$$
\sup _{h \in K} \sup _{\eta \in \mathcal{H}^{N}}\left|A_{n}(h, \eta)\right| \lesssim \sup _{h \in K} \sup _{\eta \in \mathcal{H}^{N}}\left|\frac{1}{\sqrt{n}}\left(\dot{\ell}_{\theta_{n}(h), \eta}^{(n)}-\dot{\ell}_{\theta_{0}, \eta}^{(n)}\right)^{o}\right| .
$$

For fixed $h \in K$ and $\eta \in \mathcal{H}^{N}, n^{-1 / 2}\left(\dot{\ell}_{\theta_{n}(h), \eta}^{(n)}-\dot{\ell}_{\theta_{0}, \eta}^{(n)}\right)^{o}$ converges to zero in probability because its mean is zero and its variance is bounded by,

$$
\begin{aligned}
\frac{1}{n} \sum_{i=1}^{n} P_{0}\left|\dot{\ell}_{\theta_{n}(h), \eta, i}-\dot{\ell}_{\theta_{0}, \eta, i}\right|^{2} & \\
& \lesssim \frac{1}{n} \sum_{i=1}^{n} P_{0}\left|s_{\eta}\left(X_{i}-\theta_{n}(h)^{T} Z_{i}\right)-s_{\eta}\left(X_{i}-\theta_{0}^{T} Z_{i}\right)\right|^{2} \\
& \leq \frac{1}{n} \sum_{i=1}^{n}\left|\left(\theta_{n}(h)-\theta_{0}\right)^{T} Z_{i}\right|^{2} \cdot P_{\eta_{0}} Q^{2} \lesssim \frac{P_{\eta_{0}} Q^{2}}{n}
\end{aligned}
$$

which converges to zero as $n \rightarrow \infty$. In turn, the pointwise convergence of $n^{-1 / 2}\left(\dot{\ell}_{\theta_{n}(h), \eta}^{(n)}-\dot{\ell}_{\theta_{0}, \eta}^{(n)}\right)^{o}$ to zero implies uniform convergence to zero of the right-hand side of (3.9), since (3.7) is asymptotically tight. Thus the supremum of $\left|A_{n}(h, \eta)\right|$ over $h \in K$ and $\eta \in \mathcal{H}^{N}$ is of order $o_{P_{0}}(1)$.

For $B_{n}(h, \eta)$, we prove in Section A.1.1 that,

$$
\sup _{\eta \in \mathcal{H}^{N}}\left|\frac{1}{n} P_{0}^{(n)}\left(\ell_{\theta, \eta}^{(n)}-\ell_{\theta_{0}, \eta}^{(n)}\right)+\frac{1}{2}\left(\theta-\theta_{0}\right)^{T} V_{n, \eta}\left(\theta-\theta_{0}\right)\right|=o\left(\left|\theta-\theta_{0}\right|^{2}\right)
$$

as $\theta \rightarrow \theta_{0}$. Consequently, the supremum of $B_{n}(h, \eta)$ over $h \in K$ and $\eta \in \mathcal{H}^{N}$ converges to zero.

\subsection{Proof of Condition A}

For given $\eta, \eta_{0}$, let $d_{2}$ be the metric on $\mathcal{H}$ defined by,

$$
d_{2}^{2}\left(\eta, \eta_{0}\right)=P_{\eta_{0}}\left(s_{\eta}-s_{\eta_{0}}\right)^{2}
$$

In Section A.1.2, it is shown that,

$$
\lim _{n \rightarrow \infty} \sup _{\eta \in \mathcal{H}_{n}} d_{2}\left(\eta, \eta_{0}\right)=0
$$

Let $a \in \mathbb{R}^{p}$ be a non-zero vector and let $\sigma_{n}^{2}=a^{T} \mathbf{Z}_{n} a$. Because $\rho_{\min }\left(\mathbf{Z}_{n}\right)$ is bounded away from zero in the tail by assumption, $\sigma_{n}^{2}$ is bounded away from zero for large enough $n$, and so the scaled process,

$$
\left\{\frac{a^{T}}{\sqrt{n} \sigma_{n}}\left(\dot{\ell}_{\theta_{0}, \eta}^{(n)}-P_{0}^{(n)} \dot{\ell}_{\theta_{0}, \eta}^{(n)}\right): \eta \in \mathcal{H}^{N}\right\}
$$

is asymptotically tight by the asymptotic tightness of (3.7). Furthermore, it converges weakly (in the space of bounded functions with the uniform norm) to a tight Gaussian 
process because it coverges marginally to a Gaussian distribution by the Lindberg-Feller theorem. To see this, the variance of $(3.13)$ for fixed $\eta$ is equal to $P_{\eta_{0}} s_{\eta}^{2}$ for every $n$. In addition,

$$
\begin{aligned}
\frac{1}{n \sigma_{n}^{2}} \sum_{i=1}^{n} P_{0}\left|a^{T} \dot{\ell}_{\theta_{0}, \eta, i}\right|^{2} 1_{\left\{\left|a^{T} \dot{\ell}_{\theta_{0}, \eta, i}\right|>\sqrt{n} \sigma_{n} \epsilon\right\}} & \\
= & \frac{1}{n \sigma_{n}^{2}} \sum_{i=1}^{n}\left|a^{T} Z_{i}\right|^{2} P_{\eta_{0}} s_{\eta}^{2} 1_{\left\{\left|s_{\eta}\right| \geq \sqrt{n} \epsilon \sigma_{n} /\left|a^{T} Z_{i}\right|\right\}} \\
& \lesssim \frac{1}{n} \sum_{i=1}^{n} P_{\eta_{0}} s_{\eta}^{2} 1_{\left\{\left|s_{\eta}\right| \geq \sqrt{n} \epsilon \sigma_{n} /\left|a^{T} Z_{i}\right|\right\}} \leq P_{\eta_{0}} s_{\eta}^{2} 1_{\left\{\left|s_{\eta}\right| \gtrsim \sqrt{n} \epsilon\right\}}=o(1),
\end{aligned}
$$

for every $\epsilon>0$ and large enough $n$. By the weak convergence of (3.13) to a tight Gaussian process, (3.13) is uniformly $d_{2}$-equicontinuous in probability (see Section 1.5 of [33]), because,

$$
P_{0}\left[\frac{a^{T}}{\sqrt{n} \sigma_{n}}\left(\dot{\ell}_{\theta_{0}, \eta}^{(n)}-\dot{\ell}_{\theta_{0}, \eta^{\prime}}^{(n)}\right)\right]^{2}=\frac{1}{n \sigma_{n}^{2}} \sum_{i=1}^{n} a^{T} Z_{i} Z_{i}^{T} a P_{\eta_{0}}\left(s_{\eta}-s_{\eta^{\prime}}\right)^{2}=d_{2}^{2}\left(s_{\eta}, s_{\eta^{\prime}}\right),
$$

for every $n \geq 1$. Since $P_{0}^{(n)} \dot{\ell}_{\theta_{0}, \eta}^{(n)}=0$ for every $\eta \in \mathcal{H}^{N}$, by the definition of asymptotic equicontinuity, we have,

$$
\sup \left\{\left|\frac{a^{T}\left(\dot{\ell}_{\theta_{0}, \eta}^{(n)}-\dot{\ell}_{\theta_{0}, \eta_{0}}^{(n)}\right)}{\sigma_{n}}\right|: d_{2}\left(\eta, \eta_{0}\right)<\delta_{n}, \eta \in \mathcal{H}^{N}\right\}=o_{P_{0}}\left(n^{1 / 2}\right),
$$

for every $\delta_{n} \downarrow 0$. Since $\sigma_{n}$ is bounded away from zero for large $n$ and $a$ is arbitrary, (3.12) implies 2.2 .

For (2.3), note that,

$$
\left\|V_{n, \eta}-V_{n, \eta_{0}}\right\|=\left\|\left(v_{\eta}-v_{\eta_{0}}\right) \mathbf{Z}_{n}\right\|=\left|v_{\eta}-v_{\eta_{0}}\right| \cdot\left\|\mathbf{Z}_{n}\right\|=\rho_{\max }\left(\mathbf{Z}_{n}\right) \cdot\left|v_{\eta}-v_{\eta_{0}}\right|,
$$

and $\lim \sup _{n} \rho_{\max }\left(\mathbf{Z}_{n}\right)<\infty$ because covariates are bounded. Since,

$$
\left|v_{\eta}-v_{\eta_{0}}\right|=\left|P_{\eta_{0}}\left(s_{\eta}-s_{\eta_{0}}\right) s_{\eta_{0}}\right| \lesssim d_{2}\left(\eta, \eta_{0}\right),
$$

by the Cauchy-Schwartz inequality, we have $\left\|V_{n, \eta}-V_{n, \eta_{0}}\right\| \lesssim d_{2}\left(\eta, \eta_{0}\right)$, and thus (3.12) implies 2.3.

Finally, since $v_{\eta_{0}}>0, \liminf _{n} \rho_{\min }\left(\mathbf{Z}_{n}\right)>0$ and $\sup _{i \geq 1}\left|Z_{i}\right| \leq L, 2.4$ holds trivially because $V_{n, \eta}=v_{\eta} \mathbf{Z}_{n}$.

\subsection{Proof of Condition B}

We need the following lemma, the proof of which is found in Section A.1.3.

Lemma 3.1. Under the conditions in Theorem 3.1, there exists $K>0$ such that for every sufficiently small $\epsilon>0$ and large enough $n, \eta \in \mathcal{H}_{n}$ and $h_{n}\left((\theta, \eta),\left(\theta_{0}, \eta_{0}\right)\right)<\epsilon$ imply $\left|\theta-\theta_{0}\right|<K \epsilon$ and $h\left(\eta, \eta_{0}\right)<K \epsilon$. 
Under the conditions in Theorem 3.1, it is well known (see Theorem 4 of [14]) that,

$$
P_{0}^{(n)} \Pi\left((\theta, \eta) \in \Theta_{n, 1} \times \mathcal{H}_{n, 1}: h_{n}\left((\theta, \eta),\left(\theta_{0}, \eta_{0}\right)\right) \leq M_{n} \epsilon_{n} \mid X^{(n)}\right) \rightarrow 1
$$

for every $M_{n} \rightarrow \infty$. Thus Lemma 3.1 implies (2.5).

For (2.6), let $\epsilon>0$ be a sufficiently small constant and $\left(M_{n}\right)$ be a real sequence such that $M_{n} \rightarrow \infty$ and $M_{n} / \sqrt{n} \rightarrow 0$. Also, let $\Theta_{n}=\left\{\theta \in \Theta_{n, 1}: M_{n} / \sqrt{n}<\left|\theta-\theta_{0}\right| \leq \epsilon\right\}$. Since,

$$
\begin{aligned}
& \Pi\left(\sqrt{n}\left|\theta-\theta_{0}\right|>M_{n} \mid X^{(n)}\right) \\
& \quad=\Pi\left(\left|\theta-\theta_{0}\right|>\epsilon \mid X^{(n)}\right)+\int \Pi\left(\theta \in \Theta_{n} \mid \eta, X^{(n)}\right) d \Pi\left(\eta \mid X^{(n)}\right) \\
& \quad \leq \Pi\left(\left|\theta-\theta_{0}\right|>\epsilon \mid X^{(n)}\right)+\sup _{\eta \in \mathcal{H}_{n}} \Pi\left(\theta \in \Theta_{n} \mid \eta, X^{(n)}\right)+\Pi\left(\eta \in \mathcal{H}_{n}^{c} \mid X^{(n)}\right),
\end{aligned}
$$

and $\Pi\left(\left|\theta-\theta_{0}\right|>\epsilon \mid X^{(n)}\right) \vee \Pi\left(\eta \in \mathcal{H}_{n}^{c} \mid X^{(n)}\right)$ converges to 0 in $P_{0}^{(n)}$-probability due to (3.14) with Lemma 3.1, it suffices to show that,

$$
\sup _{\eta \in \mathcal{H}_{n}} \Pi\left(\theta \in \Theta_{n} \mid \eta, X^{(n)}\right) \rightarrow 0,
$$

in $P_{0}^{(n)}$-probability. Note that,

$$
\Pi\left(\theta \in \Theta_{n} \mid \eta, X^{(n)}\right)=\frac{\int_{\Theta_{n}} p_{\theta, \eta}^{(n)} / p_{\theta_{0}, \eta}^{(n)}\left(X^{(n)}\right) d \Pi_{\Theta}(\theta)}{\int p_{\theta, \eta}^{(n)} / p_{\theta_{0}, \eta}^{(n)}\left(X^{(n)}\right) d \Pi_{\Theta}(\theta)},
$$

by Bayes's rule. In Section A.1.4, we prove that we can choose $C>C_{1}>0$ and $C_{2}>0$ such that,

$$
P_{0}^{(n)}\left(A_{n} \cap B_{n}\right) \rightarrow 1
$$

where,

$$
\begin{aligned}
& A_{n}=\left\{\inf _{\eta \in \mathcal{H}_{n}} \int_{\Theta} \frac{p_{\theta, \eta}^{(n)}}{p_{\theta_{0}, \eta}^{(n)}} d \Pi_{\Theta}(\theta) \geq C_{2}\left(\frac{M_{n}}{\sqrt{n}}\right)^{p} e^{-C_{1} M_{n}^{2}}\right\}, \\
& B_{n}=\left\{\sup _{M_{n}<|h|<\epsilon \sqrt{n}} \sup _{\eta \in \mathcal{H}_{n}} \frac{\left.p_{\theta_{n}(h), \eta}^{(n)} e^{C|h|^{2}} \leq 1\right\} .}{p_{\theta_{0}, \eta}^{(n)}},\right.
\end{aligned}
$$

The remainder of the proof is similar to that of [22]. Let $\Omega_{n}=A_{n} \cap B_{n}$,

$$
\Theta_{n, j}=\left\{\theta_{n}(h) \in \Theta_{n}: j M_{n} \leq|h|<(j+1) M_{n}\right\},
$$

and $J$ be the minimum among $j$ 's satisfying $(j+1) M_{n} / \sqrt{n}>\epsilon$. Since $\Pi_{\Theta}$ is thick at $\theta_{0}$ and $\epsilon$ is sufficiently small,

$$
\Pi_{\Theta}\left(\Theta_{n, j}\right) \leq D \cdot\left((j+1) M_{n} / \sqrt{n}\right)^{p},
$$


for some constant $D>0$. Then on $\Omega_{n}$,

$$
\begin{aligned}
\sup _{\eta \in \mathcal{H}_{n}} \Pi\left(\theta \in \Theta_{n} \mid \eta, X^{(n)}\right) & \leq \frac{e^{C_{1} M_{n}^{2}}}{C_{2}\left(M_{n} / \sqrt{n}\right)^{p}} \sup _{\eta \in \mathcal{H}_{n}} \int_{\Theta_{n}} \frac{p_{\theta, \eta}^{(n)}}{p_{\theta_{0}, \eta}^{(n)}} d \Pi_{\Theta}(\theta) \\
& \leq \frac{e^{C_{1} M_{n}^{2}}}{C_{2}\left(M_{n} / \sqrt{n}\right)^{p}} \sum_{j=1}^{J} \Pi_{\Theta}\left(\Theta_{n, j}\right) \sup _{\theta \in \Theta_{n, j}} \sup _{\eta \in \mathcal{H}_{n}} \frac{p_{\theta, \eta}^{(n)}}{p_{\theta_{0}, \eta}^{(n)}} .
\end{aligned}
$$

Since $\sup _{\theta \in \Theta_{n, j}} \sup _{\eta \in \mathcal{H}_{n}} p_{\theta, \eta}^{(n)} / p_{\theta_{0}, \eta}^{(n)} \leq \exp \left(-C j^{2} M_{n}^{2}\right)$ on $\Omega_{n}$, we have,

$$
\sup _{\eta \in \mathcal{H}_{n}} \Pi\left(\theta \in \Theta_{n} \mid \eta, X^{(n)}\right) \leq C_{2}^{-1} D e^{C_{1} M_{n}^{2}} \sum_{j=1}^{J}(j+1)^{p} e^{-C j^{2} M_{n}^{2}},
$$

on $\Omega_{n}$. Since $C>C_{1}$, the term on the right-hand side of 3.18 converges to zero as $n \rightarrow \infty$, so we conclude that 3.15 holds.

\subsection{Examples}

Conditions in Theorem 3.1 depend particularly on the choice of prior for the nuisance parameter $\eta$. In this subsection, we verify the conditions in Theorem 3.1 for two priors: a symmetric Dirichlet mixture of normal distributions and a random series prior on a smoothness class. For a given density $p$ on $\mathbb{D}$, its symmetrization $\bar{p}$ is defined by $\bar{p}=$ $\left(p+p^{-}\right) / 2$, where $p^{-}(x)=p(-x)$ for all $x \in \mathbb{D}$. We can construct a prior on $\mathcal{H}$ by putting a prior on $p \in \widetilde{\mathcal{H}}$ and symmetrizing it, where $\widetilde{\mathcal{H}}$ is the set of every density on $\mathbb{D}$ whose symmetrization belongs to $\mathcal{H}$. Obviously, we have $\mathcal{H} \subset \widetilde{\mathcal{H}}$. In this subsection, let $\Pi_{\widetilde{\mathcal{H}}}$ be a probability measure on $\widetilde{\mathcal{H}}$ and $\Pi_{\mathcal{H}}$ be the corresponding probability measure on $\mathcal{H}$. Hellinger entropy bounds and prior concentration rates around KL neighborhoods are well known for various choices of $\Pi_{\widetilde{\mathcal{H}}}$, so the following lemma is useful to prove (3.5).

Lemma 3.2. For a subset $\widetilde{\mathcal{H}}_{0}$ of $\widetilde{\mathcal{H}}$ containing $\eta_{0}$, suppose that there exists a function $\widetilde{Q}$ such that $\sup _{\eta \in \widetilde{\mathcal{H}}_{0}} P_{\eta} \widetilde{Q}^{2}<\infty$, and for every $x$ and sufficiently small $y$,

$$
\sup _{\eta \in \widetilde{\mathcal{H}}_{0}}\left|\frac{\log \eta(x+y)-\log \eta(x)}{y}\right| \leq \widetilde{Q}(x) .
$$

Furthermore, assume that for large enough n,

$$
\begin{aligned}
& \log N\left(\widetilde{\epsilon}_{n}, \widetilde{\mathcal{H}}_{n, 1}, h\right) \lesssim n \widetilde{\epsilon}_{n}^{2}, \\
& \log \Pi_{\widetilde{\mathcal{H}}}\left(\left\{\eta \in \widetilde{\mathcal{H}}: K\left(\eta_{0}, \eta\right) \leq \widetilde{\epsilon}_{n}^{2}, V\left(\eta_{0}, \eta\right) \leq \widetilde{\epsilon}_{n}^{2}\right\}\right) \gtrsim-n \widetilde{\epsilon}_{n}^{2}, \\
& \log \Pi_{\widetilde{\mathcal{H}}}\left(\widetilde{\mathcal{H}}_{n, 2}\right) \leq-\frac{5}{2} n \widetilde{\epsilon}_{n}^{2} M_{n}^{2},
\end{aligned}
$$

for some partition $\widetilde{\mathcal{H}}=\widetilde{\mathcal{H}}_{n, 1} \cup \widetilde{\mathcal{H}}_{n, 2}$ with $\eta_{0} \in \widetilde{\mathcal{H}}_{n, 1} \subset \widetilde{\mathcal{H}}_{0}$ and sequences $\widetilde{\epsilon}_{n} \rightarrow 0, M_{n} \rightarrow \infty$ with $\widetilde{\epsilon}_{n} \gtrsim n^{-1 / 2} \log n$. If $\Theta$ is compact and $\sup _{i \geq 1}\left|Z_{i}\right| \leq L$, then, for any $\Pi_{\Theta}$ that is thick at $\theta_{0}$, the product prior $\Pi_{\Theta} \times \Pi_{\mathcal{H}}$ satisfies (3.5) with some $\mathcal{H}_{n, 1} \subset \mathcal{H}_{0}, \Theta_{n, 1}=\Theta$ and $\epsilon_{n}=\widetilde{\epsilon}_{n} M_{n}$, where $\mathcal{H}_{0}$ is the set of symmetrizations of $p \in \widetilde{\mathcal{H}}_{0}$. 
Proof. For any pair of densities $p$ and $q$ on $\mathbb{D}$, it is shown in Section A.1.5 that,

$$
\begin{aligned}
h(\bar{p}, \bar{q}) & \leq \sqrt{2} h(p, q), \quad K(\bar{p}, \bar{q}) \leq K(\bar{p}, q), \\
V(\bar{p}, \bar{q}) & \leq 4\left(V(\bar{p}, q)+K^{2}(\bar{p}, q)\right),
\end{aligned}
$$

It is also shown in Section A.1.6 that there exist constants $C>0$ and $\epsilon>0$ such that,

$$
\begin{aligned}
h\left(p_{\theta_{1}, \eta_{1}, i}, p_{\theta_{2}, \eta_{2}, i}\right) & \leq C\left(\left|\theta_{1}-\theta_{2}\right|+h\left(\eta_{1}, \eta_{2}\right)\right), \\
K\left(p_{\theta_{0}, \eta_{0}, i}, p_{\theta, \eta, i}\right) & \leq C\left(\left|\theta-\theta_{0}\right|+K\left(\eta_{0}, \eta\right)\right), \\
V\left(p_{\theta_{0}, \eta_{0}, i}, p_{\theta, \eta, i}\right) & \leq C\left(\left|\theta-\theta_{0}\right|^{2}+V\left(\eta_{0}, \eta\right)+K^{2}\left(\eta_{0}, \eta\right)\right),
\end{aligned}
$$

for all $\eta_{1}, \eta_{2}, \eta \in \mathcal{H}_{0}, i \geq 1$ and $\theta_{1}, \theta_{2}, \theta$ with $\left|\theta_{1}-\theta_{2}\right| \vee\left|\theta-\theta_{0}\right|<\epsilon$.

Let $\mathcal{H}_{n, 1}$ be the set of symmetrizations of $p \in \widetilde{\mathcal{H}}_{n, 1}$. By the first inequalities of 3.21 and $(3.22)$, there is a $C_{1}>0$ such that for large enough $n$,

$$
\begin{aligned}
\log N\left(\epsilon_{n} / 36,\right. & \left.\Theta_{n, 1} \times \mathcal{H}_{n, 1}, h_{n}\right) \\
& \lesssim \log N\left(C_{1} \epsilon_{n}, \Theta_{n, 1},|\cdot|\right)+\log N\left(C_{1} \epsilon_{n}, \widetilde{\mathcal{H}}_{n, 1}, h\right) \\
& \lesssim \log \epsilon_{n}^{-1}+n \widetilde{\epsilon}_{n}^{2} \leq n \epsilon_{n}^{2},
\end{aligned}
$$

where the last inequality follows from $\epsilon_{n}>\widetilde{\epsilon}_{n} \gtrsim n^{-1 / 2} \log n$, so $\log \epsilon_{n}^{-1} \leq \log \left(n^{1 / 2} / \log n\right) \leq$ $\log n=o\left(n \epsilon_{n}^{2}\right)$. The second and third inequalities of (3.21) and (3.22), with $p=\bar{p}=\eta_{0}$, imply that there exists a constant $C_{2}>0$ such that,

$$
\begin{aligned}
& \log \Pi\left(B_{n}\left(\epsilon_{n}\right)\right) \geq \log \Pi_{\widetilde{\mathcal{H}}}\left(\left\{\eta \in \widetilde{\mathcal{H}}: K\left(\eta_{0}, \eta\right) \leq C_{2} \epsilon_{n}^{2}, V\left(\eta_{0}, \eta\right) \leq C_{2} \epsilon_{n}^{2}\right\}\right) \\
& \quad+\log \Pi_{\Theta}\left(\left\{\theta:\left|\theta-\theta_{0}\right| \leq C_{2} \epsilon_{n}^{2}\right\}\right) \\
& \geq \log \Pi_{\widetilde{\mathcal{H}}}\left(\left\{\eta \in \widetilde{\mathcal{H}}: K\left(\eta_{0}, \eta\right) \leq \widetilde{\epsilon}_{n}^{2}, V\left(\eta_{0}, \eta\right) \leq \widetilde{\epsilon}_{n}^{2}\right\}\right) \\
& \quad+\log \Pi_{\Theta}\left(\left\{\theta:\left|\theta-\theta_{0}\right| \leq \widetilde{\epsilon}_{n}^{2}\right\}\right) \\
& \geq \quad-n \widetilde{\epsilon}_{n}^{2}+\log \left(\widetilde{\epsilon}_{n}^{2}\right) \gtrsim-n \widetilde{\epsilon}_{n}^{2}-\log n \geq-n \epsilon_{n}^{2} / 4,
\end{aligned}
$$

for large enough $n$. Finally, since,

$$
\log \left(\Pi_{\mathcal{H}}\left(\mathcal{H}_{n, 2}\right)\right) \leq \log \left(\Pi_{\widetilde{\mathcal{H}}}\left(\widetilde{\mathcal{H}}_{n, 2}\right)\right) \leq-\frac{5}{2} n \epsilon_{n}^{2}
$$

the proof is complete.

\subsubsection{Symmetric Dirichlet mixtures of normal distributions}

We consider a symmetrized Dirichlet process mixture of normal densities for the prior of $\eta$. Dirichlet process mixture priors are popular and the asymptotic behavior of the posterior distribution is well-studied. A random density $\eta$ is said to follow a Dirichlet process mixture of normal densities [24] if $\eta(x)=\int \phi_{\sigma}(x-z) d F(z, \sigma)$, where $F \sim \operatorname{DP}(\alpha, H)$ and $\phi_{\sigma}$ is the density of the normal distribution with mean 0 and variance $\sigma^{2}$. Here, $\operatorname{DP}(\alpha, H)$ denotes the Dirichlet process with precision $\alpha>0$ and mean probability measure $H$ on $\mathbb{R} \times(0, \infty)[10]$. 
For given positive numbers $\sigma_{1}, \sigma_{2}$, and $M$ with $\sigma_{1}<\sigma_{2}$, let $\mathcal{F}$ be the set of all distribution functions supported on $[-M, M] \times\left[\sigma_{1}, \sigma_{2}\right]$, and let $\widetilde{\mathcal{H}}_{0}$ be the set of all densities $\eta$ on $\mathbb{R}$ of the form $\eta(x)=\int \phi_{\sigma}(x-z) d F(z, \sigma)$ for $F \in \tilde{\mathcal{F}}$. Then it is easy to show that $\mathcal{H}_{0}$, the symmetrization of $\widetilde{\mathcal{H}}_{0}$, is the set of all $\eta \in \widetilde{\mathcal{H}}_{0}$, where $F \in \mathcal{F}$ with $d F(z, \sigma)=d F(-z, \sigma)$. If $F \sim \operatorname{DP}(\alpha, H)$, where $H$ has a positive and continuous density supported on $[-M, M] \times\left[\sigma_{1}, \sigma_{2}\right]$, the corresponding Dirichlet process mixture prior and its symmerization, denoted by $\Pi_{\widetilde{\mathcal{H}}}$ and $\Pi_{\mathcal{H}}$, respectively, have full support on $\widetilde{\mathcal{H}}_{0}$ and $\mathcal{H}_{0}$ relative to the Hellinger topology.

Corollary 3.1. Suppose that $\sup _{i \geq 1}\left|Z_{i}\right| \leq L$ and $\liminf _{n} \rho_{\min }\left(\mathbf{Z}_{n}\right)>0$. With the symmetrized Dirichlet process mixture prior described above for $\eta$, the BvM theorem holds for the linear regression model provided that $\eta_{0} \in \mathcal{H}_{0}$ and that $\Pi_{\Theta}$ is compactly supported and thick at $\theta_{0}$.

Proof. We may assume that $\Theta$ is compact, and let $\Theta_{n, 1}=\Theta$. It is trivial that $v_{\eta_{0}}>0$. The first and second derivatives of the map $x \mapsto \ell_{\eta}(x)$ are of orders $O(x)$ and $O\left(x^{2}\right)$, respectively, as $x \rightarrow \infty$ (see lemma 3.2.3 of [8] for details), and both bounds can be chosen independently of $\eta$. Consequently, condition (3.6) holds with $Q(x)=O\left(x^{2}\right)$ as $|x| \rightarrow \infty$, and $\sup _{\eta \in \widetilde{\mathcal{H}}_{0}} P_{\eta} Q^{2}<\infty$. The proof of Theorem 6.2 in [13] implies that 3.20 ] holds with $\widetilde{\mathcal{H}}_{n, 1}=\widetilde{\mathcal{H}}_{0}, \widetilde{\epsilon}_{n}=n^{-1 / 2}(\log n)^{3 / 2}$ and any $M_{n} \rightarrow \infty$. Thus, 3.5 hold with $\epsilon_{n}=n^{-1 / 2}(\log n)^{2}$ and $\mathcal{H}_{n, 1}=\mathcal{H}_{0}$.

What remains to prove for the BvM assertion is asymptotic tightness c.f. (3.7), which is implied if for every $a \in \mathbb{R}^{p}$ and sufficiently small $\epsilon>0$, the stochastic process,

$$
\left\{(\theta, \eta) \mapsto \frac{a^{T}}{\sqrt{n}} \sum_{i=1}^{n}\left(\dot{\ell}_{\theta, \eta, i}-P_{0} \dot{\ell}_{\theta, \eta, i}\right): \theta \in B_{\epsilon}, \eta \in \mathcal{H}_{0}\right\},
$$

is asymptotically tight, where $B_{\epsilon}$ is the open ball of radius $\epsilon$ centred on $\theta_{0}$. In Section A.1.7. we prove the asymptotic tightness of (3.23) using the bracketing central limit theorem.

The symmetrized Dirichlet process mixture prior considered in this subsection is restricted, in that the mixing distribution $F$ is supported on $[-M, M] \times\left[\sigma_{1}, \sigma_{2}\right]$. This restriction plays only a technical role (to prove (3.6) and (3.7) ) and it is expected that, with some additional effort, the results could be extended to arbitrarily small $\sigma$ 's and arbitraily large $M$.

\subsubsection{Random series prior}

Let $W$ be a random function on $[-1 / 2,1 / 2]$ defined as a series $W(\cdot)=\sum_{j=1}^{\infty} j^{-\alpha} C_{j} b_{j}(\cdot)$, where $b_{1}(t)=1, b_{2 j}(t)=\cos (2 \pi j t), b_{2 j+1}(t)=\sin (2 \pi j t)$ and $C_{j}$ 's are i.i.d. random variables drawn from a density supported on $[-M, M]$ that is continuous and bounded away from zero. We shall impose smoothness through the requirement that $\alpha$ be greater than 3 , so that the series is well defined as a continuous real-valued function on $[-1 / 2,1 / 2]$ with the first and second derivatives that are bounded uniformly by a constant. Let $\mathcal{W}$ be the 
set of all functions $w:[-1 / 2,1 / 2] \rightarrow \mathbb{R}$ of the form $w(\cdot)=\sum_{j} a_{j} b_{j}(\cdot)$ for some sequence $\left(a_{1}, a_{2}, \ldots\right)$ with $j^{\alpha}\left|a_{j}\right| \leq M$ for all $j$. Let $\widetilde{\mathcal{H}}_{0}$ denote the set of densities $p_{w}$, where $w \in \mathcal{W}$ and,

$$
p_{w}(x)=\frac{e^{w(x)}}{\int_{-1 / 2}^{1 / 2} e^{w(y)} d y},
$$

for every $x \in \mathbb{D}=(-1 / 2,1 / 2)$. Let $\mathcal{H}_{0}$ denote the associated space of symmetrized $\bar{p}_{w}$. Let $\Pi_{\widetilde{\mathcal{H}}}$ and $\Pi_{\mathcal{H}}$ be the laws of random densities $p_{W}$ and $\bar{p}_{W}$, respectively.

Corollary 3.2. Suppose that $\sup _{i \geq 1}\left|Z_{i}\right| \leq L$ and $\liminf _{n} \rho_{\min }\left(\mathbf{Z}_{n}\right)>0$. If $\alpha>3$, $\eta_{0} \in \mathcal{H}_{0}, v_{\eta_{0}}>0$, and $\Pi_{\Theta}$ is compactly supported and thick at $\theta_{0}$, then the random series prior $\Pi_{\mathcal{H}}$ for $\eta$ leads to a posterior for $\theta$ that satisfies the BvM assertion (2.7) in the linear regression model.

Proof. We may assume that $\Theta$ is compact. Let $W$ be the random function defined above, and let $w_{0}(\cdot)=\sum_{j=1}^{\infty} j^{-\alpha} c_{0, j} b_{j}(\cdot)$ such that $\eta_{0}(x) \propto e^{w_{0}(x)}+e^{w_{0}(-x)}$. One verifies easily that the KL-divergence $K$, KL-variation $V$ and the square Hellinger distance $h^{2}$, for densities $p_{w}(\cdot) \propto e^{w(\cdot)}$ are bounded by the square of the uniform norm of the difference between the exponents $w$. Therefore by Lemma 3.2, conditions 3.5) (with $\Theta_{n, 1}=\Theta$ and $\left.\mathcal{H}_{n, 1}=\mathcal{H}_{0}\right)$ hold for some $\left(\epsilon_{n}\right)$ under the two conditions: $\Pi_{\mathcal{W}}\left\{\left\|W-w_{0}\right\|_{\infty}<\epsilon\right\}>0$ and $N\left(\epsilon, \mathcal{W},\|\cdot\|_{\infty}\right)<\infty$ for every $\epsilon>0$, where $\|\cdot\|_{\infty}$ is the uniform norm and $\Pi_{\mathcal{W}}$ is the law of $W$. Since $\mathcal{W}$ is totally bounded with respect to $\|\cdot\|_{\infty}$ by the Arzelà-Ascoli theorem, the condition $N\left(\epsilon, \mathcal{W},\|\cdot\|_{\infty}\right)<\infty$ is satisfied. For given $\epsilon>0$, there exists an integer $J$ such that $M \cdot \sum_{j=J+1}^{\infty} j^{-\alpha}<\epsilon / 4$. Since each random variable $C_{j}$ has a positive and continuous density at $c_{0, j}$ for $j \leq J$, we have $\Pi_{\mathcal{W}}(A)>0$ for $A=\left\{\max _{j \leq J}\left|C_{j}-c_{0, j}\right|<\right.$ $\left.\epsilon /\left(2 \sum_{j=1}^{\infty} j^{-\alpha}\right)\right\}$. Since $\left\|W-w_{0}\right\|_{\infty}<\epsilon$ on $A$, we have $\Pi_{\mathcal{W}}\left\{\left\|W-w_{0}\right\|_{\infty}<\epsilon\right\}>0$.

Note that (3.6) is trivially satisfied with a constant function $Q$. In Section A.1.8, we prove the asymptotic tightness of (3.7), which completes the proof.

\section{Efficiency in the linear mixed effect model}

In this section, we consider the linear mixed effect model,

$$
X_{i j}=\theta^{T} Z_{i j}+b_{i}^{T} W_{i j}+\epsilon_{i j}, \quad \text { for } i=1, \ldots, n \text { and } j=1, \ldots, m_{i},
$$

where the covariates $Z_{i j} \in \mathbb{R}^{p}$ and $W_{i j} \in \mathbb{R}^{q}$ are non-random, the error $\epsilon_{i j}$ 's form an i.i.d. sequence drawn from a distribution with density $f$ and the random effect coefficients $b_{i}$ are i.i.d. from a distribution $G$. The nuisance parameter $\eta=(f, G)$ takes its values in the space $\mathcal{H}=\mathcal{F} \times \mathcal{G}$, where the first factor $\mathcal{F}$ denotes the class of continuously differentiable densities supported on $\mathbb{D}=(-r, r)$ for some $r \in(0, \infty]$ with $f(x)>0$ and $f(x)=f(-x)$ for all $x \in \mathbb{D}$ and $\mathcal{G}$ is the class of symmetric distributions supported on $\left[-M_{b}, M_{b}\right]^{q}$ for some $M_{b}>0$. The true value of the nuisance is denoted by $\eta_{0}=\left(f_{0}, G_{0}\right)$. We write 
$X_{i}=\left(X_{i 1}, \ldots, X_{i m_{i}}\right)^{T}$, and similarly, $Z_{i} \in \mathbb{R}^{p \times m_{i}}$ and $W_{i} \in \mathbb{R}^{q \times m_{i}}$. As in the linear regression model, we assume that,

$$
\left|Z_{i j}\right| \leq L \quad \text { and } \quad\left|W_{i j}\right| \leq L, \quad \text { for all } i \text { and } j
$$

Define,

$$
p_{\theta, \eta, i}(x)=\int \prod_{j=1}^{m_{i}} f\left(x_{j}-\theta^{T} Z_{i j}-b_{i}^{T} W_{i j}\right) d G\left(b_{i}\right),
$$

where $x=\left(x_{1}, \ldots, x_{m_{i}}\right)^{T} \in \mathbb{R}^{m_{i}}$. Quantities denoted by $p_{\theta, \eta}^{(n)}, \ell_{\theta, \eta, i}, \dot{\ell}_{\theta, \eta, i}$ and $\dot{\ell}_{\theta, \eta}^{(n)}$ are defined and used in the same way as in Section 3. The design matrix $\mathbf{Z}_{n}$ is defined by $\mathbf{Z}_{n}=n^{-1} \sum_{i=1}^{n} Z_{i} Z_{i}^{T}$. For technical reasons and notational convenience, we assume that there exists an integer $m$ such that $m_{i}=m$ for all $i$, but proofs below can be extended to general cases without much hamper.

For $y=\left(y_{1}, \ldots, y_{m}\right)^{T} \in \mathbb{R}^{m}$ and $w=\left(w_{1}, \ldots, w_{m}\right) \in[-L, L]^{q \times m}$, define,

$$
\psi_{\eta}(y \mid w)=\int \prod_{j=1}^{m} f\left(y_{j}-b^{T} w_{j}\right) d G(b),
$$

and $\ell_{\eta}(y \mid w)=\log \psi_{\eta}(y \mid w)$. Let $s_{\eta}(y \mid w)=-\partial \ell_{\eta}(y \mid w) / \partial y \in \mathbb{R}^{m}$. Then it can be easily shown that $\dot{\ell}_{\theta, \eta, i}(x)=Z_{i} s_{\eta}\left(x-Z_{i}^{T} \theta \mid W_{i}\right) \in \mathbb{R}^{p}$. Furthermore, let $\Psi_{\eta}^{w}(\cdot)$ denote the probability measure on $\mathbb{R}^{m}$ with density $y \mapsto \psi_{\eta}(y \mid w)$. The metric $h_{n}$ on $\Theta \times \mathcal{H}$ is defined as in (3.3). With slight abuse of notation, we also use $h_{n}$ as a metric on $\mathcal{H}$ defined as $h_{n}\left(\eta_{1}, \eta_{2}\right)=h_{n}\left(\left(\theta_{0}, \eta_{1}\right),\left(\theta_{0}, \eta_{2}\right)\right)$. Let,

$$
d_{w}^{2}\left(\eta_{1}, \eta_{2}\right)=\int\left|s_{\eta_{1}}(y \mid w)-s_{\eta_{2}}(y \mid w)\right|^{2} d \Psi_{\eta_{0}}^{w}(y)
$$

Define $B_{n}(\epsilon)$ and $V_{n, \eta}$ as in $(3.2)$ and (3.4), respectively. It can be easily shown that,

$$
V_{n, \eta}=\frac{1}{n} \sum_{i=1}^{n} Z_{i} v_{\eta}\left(W_{i}\right) Z_{i}^{T},
$$

where $v_{\eta}(w)$ is the $m \times m$ matrix defined as,

$$
v_{\eta}(w)=\int s_{\eta}(y \mid w) s_{\eta_{0}}(y \mid w)^{T} d \Psi_{\eta_{0}}^{w}(y) .
$$

To prove the BvM assertion in the linear mixed effect model, we need a condition to ensure that $\sup _{i \geq 1} h\left(\psi_{\eta_{n}}\left(\cdot \mid W_{i}\right), \psi_{\eta_{0}}\left(\cdot \mid W_{i}\right)\right) \rightarrow$ as $h_{n}\left(\eta_{n}, \eta_{0}\right) \rightarrow 0$. For this purpose, we define $N_{n, \epsilon}(u)$ to be the number of $W_{i j}$ 's with $\left|W_{i j}-u\right|<\epsilon$, and assume that, for every (fixed) $\epsilon>0$ and $u \in \mathbb{R}^{q}$,

$$
N_{n, \epsilon}(u)=0 \text { for all } n, \text { or } \liminf _{n} n^{-1} N_{n, \epsilon}(u)>0 .
$$

Condition 4.3 is easily satisfied, for example when $W_{i j}$ 's are i.i.d. realization from any distribution. 
Theorem 4.1. Suppose that $\liminf _{n} \rho_{\min }\left(\mathbf{Z}_{n}\right)>0, \rho_{\min }\left(v_{\eta_{0}}(w)\right)>0$ for every $w, G_{0}$ is thick at $0, \Pi_{\Theta}$ is thick at $\theta_{0}$, and $w \mapsto v_{\eta_{0}}(w)$ is continuous. Also suppose that there exist a large integer $N$, a sequence $\left(\epsilon_{n}\right)$, with $\epsilon_{n} \downarrow 0$ and $n \epsilon_{n}^{2} \rightarrow \infty$, and sequences of partitions $\Theta=\Theta_{n, 1} \cup \Theta_{n, 2}, \mathcal{H}=\mathcal{H}_{n, 1} \cup \mathcal{H}_{n, 2}$ such that $\eta_{0} \in \mathcal{H}_{n, 1}$ and (3.5) holds for all $n \geq N$. For some $\bar{M}_{n} \uparrow \infty$, with $\epsilon_{n} \bar{M}_{n} \rightarrow 0$, let $\mathcal{H}_{n}=\left\{\eta \in \mathcal{H}_{n, 1}: h_{n}\left(\eta, \eta_{0}\right)<\bar{M}_{n} \epsilon_{n}\right\}$. Assume that there exists a continuous function $Q$ such that $\sup _{w} \int Q^{3}(x, w) \psi_{\eta_{0}}(x \mid w) d \mu(x)<\infty$, and,

$$
\sup _{\eta \in \mathcal{H}^{N}} \frac{\left|\ell_{\eta}(x+y \mid w)-\ell_{\eta}(x \mid w)\right|}{|y|} \vee \frac{\left|s_{\eta}(x+y \mid w)-s_{\eta}(x \mid w)\right|}{|y|} \leq Q(x, w),
$$

for all $x, w$ and small enough $|y|$, where $\mathcal{H}^{N}=\cup_{n=N}^{\infty} \mathcal{H}_{n}$. Also assume that the class of $\mathbb{R}^{2}$-valued functions,

$$
\left\{w \mapsto\left(d_{w}\left(\eta_{1}, \eta_{2}\right), h\left(\psi_{\eta_{1}}(\cdot \mid w), \psi_{\eta_{2}}(\cdot \mid w)\right)\right): \eta_{1}, \eta_{2} \in \mathcal{H}^{N}\right\}
$$

is equicontinuous, and for sufficiently small $\epsilon_{0}>0$ the stochastic process,

$$
\left\{\frac{1}{\sqrt{n}}\left(\dot{\ell}_{\theta, \eta}^{(n)}-P_{0}^{(n)} \dot{\ell}_{\theta, \eta}^{(n)}\right):\left|\theta-\theta_{0}\right|<\epsilon_{0}, \eta \in \mathcal{H}^{N}\right\}
$$

is asymptotically tight. Then, the BvM assertion (2.7) holds for the linear mixed effect model, provided that (4.1) and 4.3) hold.

The proof of Theorem 4.1 is quite similar to that of Theorem 3.1 except for some technical details. Below we follow the same line to the proof of Theorem 3.1 .

\subsection{Proof of the misspecified LAN property}

Let (3.8) define $A_{n}(h, \eta)$ and $B_{n}(h, \eta)$ again and let $K$ be a compact subset of $\mathbb{R}^{p}$. Then it suffices to prove that $A_{n}(h, \eta)$ and $B_{n}(h, \eta)$ converge in $P_{0}^{(n)}$-probability to zero uniformly over $h \in K$ and $\eta \in \mathcal{H}^{N}$. Note that the inequality (3.9) still holds. Since,

$$
\begin{aligned}
& \operatorname{Var}\left(\frac{1}{\sqrt{n}}\left(\dot{\ell}_{\theta_{n}(h), \eta}^{(n)}-\dot{\ell}_{\theta_{0}, \eta}^{(n)}\right)^{o}\right)=\frac{1}{n} \sum_{i=1}^{n} P_{0}\left|\dot{\ell}_{\theta_{n}(h), \eta, i}-\dot{\ell}_{\theta_{0}, \eta, i}\right|^{2} \\
& \quad=\frac{1}{n} \sum_{i=1}^{n} P_{0}\left|Z_{i}\left(s_{\eta}\left(X_{i}-Z_{i}^{T} \theta_{n}(h) \mid W_{i}\right)-s_{\eta}\left(X_{i}-Z_{i}^{T} \theta_{0} \mid W_{i}\right)\right)\right|^{2} \\
& \leq \frac{1}{n} \sum_{i=1}^{n}\left\|Z_{i}\right\|^{4} \cdot\left|\theta_{n}(h)-\theta_{0}\right|^{2} \cdot P_{0} Q\left(X_{i}, W_{i}\right)^{2}=o(1)
\end{aligned}
$$

$\sup _{h \in K} \sup _{\eta \in \mathcal{H}^{N}}\left|A_{n}(h, \eta)\right|=o_{P_{0}}(1)$ by asymptotic tightness of 4.6.

For $B_{n}(h, \eta)$, we prove in Section A.2.1 that,

$$
\sup _{\eta \in \mathcal{H}^{N}}\left|\frac{1}{n} P_{0}^{(n)}\left(\ell_{\theta, \eta}^{(n)}-\ell_{\theta_{0}, \eta}^{(n)}\right)+\frac{1}{2}\left(\theta-\theta_{0}\right)^{T} V_{n, \eta}\left(\theta-\theta_{0}\right)\right|=o\left(\left|\theta-\theta_{0}\right|^{2}\right),
$$

as $\theta \rightarrow \theta_{0}$. Consequently, the supremum of $B_{n}(h, \eta)$ over $h \in K$ and $\eta \in \mathcal{H}^{N}$ converges to 0 . 


\subsection{Proof of Condition A}

It is shown in Section A.2.2 that,

$$
\lim _{n \rightarrow \infty} \sup _{i \geq 1} \sup _{\eta \in \mathcal{H}_{n}} d_{W_{i}}\left(\eta, \eta_{0}\right)=0 .
$$

Note that for any $a \in \mathbb{R}^{m}$ with $|a|=1$,

$$
\begin{aligned}
& a^{T}\left(v_{\eta}(w)-v_{\eta_{0}}(w)\right) a \\
& \quad=\int a^{T}\left(s_{\eta}(x \mid w)-s_{\eta_{0}}(x \mid w)\right) s_{\eta_{0}}(x \mid w)^{T} a d \Psi_{\eta_{0}}^{w}(x) \\
& \quad \leq C \int\left|s_{\eta}(x \mid w)-s_{\eta_{0}}(x \mid w)\right|^{2} d \Psi_{\eta_{0}}^{w}(x)=C d_{w}^{2}\left(\eta, \eta_{0}\right),
\end{aligned}
$$

for some constant $C>0$ by the Cauchy-Schwartz inequality and (4.4). Thus,

$$
\lim _{n \rightarrow \infty} \sup _{i \geq 1} \sup _{\eta \in \mathcal{H}_{n}}\left\|v_{\eta}\left(W_{i}\right)-v_{\eta_{0}}\left(W_{i}\right)\right\|=0 .
$$

Since,

$$
\sup _{\eta \in \mathcal{H}_{n}}\left\|V_{n, \eta}-V_{n, \eta_{0}}\right\|=\sup _{\eta \in \mathcal{H}_{n}}\left\|\frac{1}{n} \sum_{i=1}^{n} Z_{i}\left\{v_{\eta}\left(W_{i}\right)-v_{\eta_{0}}\left(W_{i}\right)\right\} Z_{i}^{T}\right\|=o(1),
$$

which completes the proof of 2.3.

Let $a \in \mathbb{R}^{p}$ be a fixed non-zero vector. Then for any sequence $\eta_{n} \in \mathcal{H}_{n}$,

$$
\operatorname{Var}\left(\frac{a^{T}}{\sqrt{n}}\left(\dot{\ell}_{\theta_{0}, \eta_{n}}^{(n)}-\dot{\ell}_{\theta_{0}, \eta_{0}}^{(n)}\right)\right)=\frac{1}{n} \sum_{i=1}^{n} a^{T} Z_{i} u_{\eta_{n}}\left(W_{i}\right) Z_{i}^{T} a
$$

where,

$$
u_{\eta}(w)=\int\left(s_{\eta}(x \mid w)-s_{\eta_{0}}(x \mid w)\right)\left(s_{\eta}(x \mid w)-s_{\eta_{0}}(x \mid w)\right)^{T} d \Psi_{\eta_{0}}^{w}(x) .
$$

Since $\left|b^{T} u_{\eta}(w) b\right| \leq d_{w}\left(\eta, \eta_{0}\right)$ for every $\eta \in \mathcal{H}^{N}$ and $b \in \mathbb{R}^{m}$ with $|b|=1$, we have $\sup _{i \geq 1}\left\|u_{\eta_{n}}\left(W_{i}\right)\right\|=o(1)$ by (4.8), and so,

$$
\frac{a^{T}}{\sqrt{n}}\left(\dot{\ell}_{\theta_{0}, \eta_{n}}^{(n)}-\dot{\ell}_{\theta_{0}, \eta_{0}}^{(n)}\right)=o_{P_{0}}(1)
$$

For given $\epsilon, \delta>0$, by asymptotic tightness of 4.6 and Theorem 1.5.6 of [33], there is a partition $\mathcal{H}^{N}=\cup_{j=1}^{J} \mathcal{H}^{(j)}$ such that,

$$
P_{0}\left(\max _{1 \leq j \leq J} \sup _{\eta_{1}, \eta_{2} \in \mathcal{H}^{(j)}}\left|\frac{a^{T}}{\sqrt{n}}\left(\dot{\ell}_{\theta_{0}, \eta_{1}}^{(n)}-\dot{\ell}_{\theta_{0}, \eta_{2}}^{(n)}\right)\right|>\epsilon\right)<\delta,
$$

for large enough $n$. We can choose sequences $\left(\eta_{n}^{(j)}\right)$ for $j=1, \ldots, J$ such that $\eta_{n}^{(j)} \in \mathcal{H}_{n}$ and for every $n \geq N$ and for a given $\eta \in \mathcal{H}_{n}$ there exists at least one $j$ such that $\eta$ and $\eta_{n}^{(j)}$ are contained in the same partition. Since,

$$
\max _{1 \leq j \leq J}\left|\frac{a^{T}}{\sqrt{n}}\left(\dot{\ell}_{\theta_{0}, \eta_{n}^{(n)}}^{(j)}-\dot{\ell}_{\theta_{0}, \eta_{0}}^{(n)}\right)\right|=o_{P_{0}}(1)
$$


we have,

$$
P_{0}\left(\sup _{\eta \in \mathcal{H}_{n}}\left|\frac{a^{T}}{\sqrt{n}}\left(\dot{\ell}_{\theta_{0}, \eta}^{(n)}-\dot{\ell}_{\theta_{0}, \eta_{0}}^{(n)}\right)\right|>2 \epsilon\right)<2 \delta,
$$

for large enough $n$. Since $a$ is an arbitrary vector, 2.2 is proved.

Since $\rho_{\min }\left(v_{\eta_{0}}(w)\right)>0$ for every $w$ and the map $w \mapsto v_{\eta_{0}}(w)$ is continuous, we have that $\inf _{w \in[-L, L]^{q \times m}} \rho_{\min }\left(v_{\eta_{0}}(w)\right)>0$. In addition, since each component of the matrix $v_{\eta_{0}}(w)$ is bounded uniformly in $w$ by the integrability condition (4.4), we have $\sup _{w \in[-L, L]^{q \times m}} \rho_{\max }\left(v_{\eta_{0}}(w)\right)<\infty$. Finally, since,

$$
0<\liminf _{n} \rho_{\min }\left(\mathbf{Z}_{n}\right) \leq \limsup _{n} \rho_{\max }\left(\mathbf{Z}_{n}\right)<\infty,
$$

2.4 is satisfied by 4.2 .

\subsection{Proof of Condition B}

We shall have need for the following lemma, the proof of which is in Section A.2.3.

Lemma 4.1. Under the conditions in Theorem 4.1, there exists $K>0$ such that for every sufficiently small $\epsilon>0$ and $\eta \in \mathcal{H}^{N}, h_{n}\left((\theta, \eta),\left(\theta_{0}, \eta_{0}\right)\right)<\epsilon$ implies $\left|\theta-\theta_{0}\right|<K \epsilon$ and $h_{n}\left(\eta, \eta_{0}\right)<K \epsilon$.

Posterior consistency of the parameter $(\theta, \eta)$ with respect to the metric $h_{n}$ is guaranteed by Theorem 4 of [14]. Thus, Lemma 4.1 implies (2.5). The proof of (2.6) for the linear mixed effect model is very similar to the analogous proof in linear regression model, as in Section 3 .

\subsection{Examples}

Let $\widetilde{\mathcal{F}}$ (resp. $\widetilde{\mathcal{G}}$ ) be the set of every $f$ (resp. $G$ ) whose symmetrization $\bar{f}$ (resp. $\bar{G}$ ) belongs to $\mathcal{F}$ (resp. $\mathcal{G}$ ), where $\bar{G}=\left(G+G^{-}\right) / 2$ with $G^{-}(A)=G(-A)$ for every measurable set $A$. For the prior of $\eta$, we consider a product measure $\Pi_{\mathcal{F}} \times \Pi_{\mathcal{G}}$, where $\Pi_{\mathcal{F}}$ and $\Pi_{\mathcal{G}}$ are the symmetrized versions of probability measures $\Pi_{\widetilde{\mathcal{F}}}$ and $\Pi_{\widetilde{\mathcal{G}}}$ on $\widetilde{\mathcal{F}}$ and $\widetilde{\mathcal{G}}$, respectively. The following lemma plays a role in the proof of Corollary 4.1 (its proof is given in Section A.2.4. Denote the Lévy-Prohorov metric between two probability measures $P_{1}, P_{2}$ is denoted by $d_{W}\left(P_{1}, P_{2}\right)$.

Lemma 4.2. Let $\mathcal{H}_{0}=\mathcal{F}_{0} \times \mathcal{G}_{0} \subset \mathcal{H}$ for some $\mathcal{F}_{0} \subset \mathcal{F}$ and $\mathcal{G}_{0} \subset \mathcal{G}$ with $f_{0} \in \mathcal{F}_{0}$ and $G_{0} \in \mathcal{G}_{0}$. Assume that there exist a continuous function $Q_{0}$ and small enough $\delta_{0}>0$ such that,

$$
\int \sup _{w} \sup _{\eta \in \mathcal{H}_{0}} Q_{0}(x, w)^{2} \psi_{\eta}(x \mid w) d \mu(x)<\infty
$$

and,

$$
\sup _{\eta \in \mathcal{H}_{0}} \frac{\left|\ell_{\eta}(x+y \mid w)-\ell_{\eta}(x \mid w)\right|}{|y|} \vee\left|\frac{\psi_{\eta_{0}}(x \mid w)}{\psi_{\eta}(x \mid w)}\right|^{\delta_{0}} \leq Q_{0}(x, w)
$$


for all $x, w$ and small enough $|y|$. Also assume that $\mathcal{F}_{0}$ is uniformly tight and,

$$
\sup _{f \in \mathcal{F}_{0}} \sup _{x} f(x) \vee|\dot{f}(x)|<\infty,
$$

where $\dot{f}$ is the derivative of $f$. Then, on $\Theta \times \mathcal{H}_{0}$,

$$
\sup _{n \geq 1} h_{n}\left(\left(\theta_{1}, \eta_{1}\right),\left(\theta_{2}, \eta_{2}\right)\right) \rightarrow 0,
$$

as $\left|\theta_{1}-\theta_{2}\right| \vee h\left(f_{1}, f_{2}\right) \vee d_{W}\left(G_{1}, G_{2}\right) \rightarrow 0$, and,

$$
\sup _{n \geq 1} \frac{1}{n} \sum_{i=1}^{n} K\left(p_{\theta_{0}, \eta_{0}, i}, p_{\theta, \eta, i}\right) \vee V\left(p_{\theta_{0}, \eta_{0}, i}, p_{\theta, \eta, i}\right) \rightarrow 0,
$$

as $\left|\theta-\theta_{0}\right| \vee h\left(f, f_{0}\right) \vee d_{W}\left(G, G_{0}\right) \rightarrow 0$.

\subsubsection{Symmetric Dirichlet mixtures of normal distributions}

Let $\Pi_{\mathcal{F}}$ denote the prior for the symmetric Dirichlet mixtures of normal distributions defined in Section 3.4.1 and let $\mathcal{F}_{0}$ be the support of $\Pi_{\mathcal{F}}$ in Hellinger metric. Let $\mathcal{G}_{0}$ be the support of a prior $\Pi_{\mathcal{G}}$ on $\mathcal{G}$ in the weak topology, and let $\mathcal{H}_{0}=\mathcal{F}_{0} \times \mathcal{G}_{0}$. The following corollary proves the BvM theorem for $\theta$.

Corollary 4.1. Assume that $\liminf _{n} \rho_{\min }\left(\mathbf{Z}_{n}\right)>0$. With the prior $\Pi_{\mathcal{H}}$ described above, the BvM theorem holds for the linear mixed regression model provided that $\eta_{0} \in \mathcal{H}_{0}, G_{0}$ is thick at 0 , and $\Pi_{\Theta}$ is compactly supported and thick at $\theta_{0}$, provided (4.1) and (4.3) hold.

Proof. We may assume that $\Theta$ is compact, and let $\Theta_{n, 1}=\Theta$ and $\mathcal{H}_{n, 1}=\mathcal{H}_{0}$ for all $n \geq 1$. It is easy to show that $\rho_{\min }\left(v_{\eta_{0}}(w)\right)>0$ for every $w$ and $w \mapsto v_{\eta_{0}}(w)$ is continuous. We prove in Section A.2.5 that

$$
\begin{aligned}
C_{1} \exp \left(-C_{2}|x|^{2}\right) & \leq \inf _{w} \inf _{\eta \in \mathcal{H}_{0}} \psi_{\eta}(x \mid w) \\
& \leq \sup _{w} \sup _{\eta \in \mathcal{H}_{0}} \psi_{\eta}(x \mid w) \leq C_{3} \exp \left(-C_{4}|x|^{2}\right)
\end{aligned}
$$

for some constants $C_{1}, C_{2}, C_{3}, C_{4}>0$ and large enough $|x|$. Also, the first and second order partial derivative of $x \mapsto \ell_{\eta}(x \mid w)$ are of order $O\left(|x|^{2}\right)$ as $|x| \rightarrow \infty$ for every $\eta \in \mathcal{H}_{0}$ and $w$, so, with $Q(x, w)=C_{5}\left(1+|x|^{2}\right)$ for some $C_{5}>0$, we have,

$$
\sup _{\eta \in \mathcal{H}_{0}} \frac{\left|\ell_{\eta}(x+y \mid w)-\ell_{\eta}(x \mid w)\right|}{|y|} \vee \frac{\left|s_{\eta}(x+y \mid w)-s_{\eta}(x \mid w)\right|}{|y|} \leq Q(x, w),
$$

for every $x, w$ and small enough $|y|$, and,

$$
\int \sup _{w} \sup _{\eta \in \mathcal{H}_{0}} Q^{3}(x, w) \psi_{\eta}(x \mid w) d \mu(x)<\infty .
$$

We next prove 3.5 with the help of Lemma 4.2 . Since $\Pi_{\Theta}\left(\Theta_{n, 1}\right)=\Pi_{\mathcal{H}}\left(\mathcal{H}_{n, 1}\right)=1$, the third inequality of $(3.5)$ holds trivially. By (4.15),

$$
\int \sup _{\eta_{1}, \eta_{2} \in \mathcal{H}_{0}}\left|\frac{\psi_{\eta_{0}}(x \mid w)}{\psi_{\eta}(x \mid w)}\right|^{2 \delta_{0}} \psi_{\eta_{2}}(x \mid w) d \mu(x)<\infty
$$


for sufficiently small $\delta_{0}>0$, so combining with 4.17), (4.10) and 4.11) hold for some $Q_{0}$. Uniform tightness of $\mathcal{F}_{0}$ and 4.12 is easily satisfied, so the conclusion of Lemma 4.2 holds. By (4.13), the first inequality of $(3.5)$ holds for some rate sequence $\epsilon_{n, 1}$ because $\Theta \times \mathcal{F}_{0} \times \mathcal{G}_{0}$ is totally bounded with respect to the product metric $|\cdot| \times h \times d_{W}$. Also, by (4.14), the second inequality of 3.5 holds for some $\epsilon_{n, 2}$ because every $|\cdot| \times h \times d_{W}$ neighborhoods of $\left(\theta_{0}, f_{0}, G_{0}\right)$ has positive prior mass. Thus, 3.5 holds with $\epsilon_{n}=\max \left\{\epsilon_{n, 1}, \epsilon_{n, 2}\right\}$.

To complete the proof, equicontinuity of 4.5 is proved in Section A.2.6 and asymptotic tightness of 4.6 in Section A.2.7. $\square$ It should be noted that the only condition for $\Pi_{\mathcal{G}}$ is that $G_{0} \in \mathcal{G}_{0}$. Thus, we can consider both parametric and nonparametric priors for $G$. For example, the multivariate normal distribution truncated on $\left[-M_{b}, M_{b}\right]^{q}$ or the symmetrized $\operatorname{DP}\left(\alpha, H_{G}\right)$ prior with a distribution $H_{G}$ on $\left[-M_{b}, M_{b}\right]^{q}$ can be used for $\Pi_{\mathcal{G}}$.

\subsubsection{Random series prior}

Let $\Pi_{\mathcal{F}}$ be the random series prior defined in Section 3.4 .2 and let $\mathcal{F}_{0}$ be the support of $\Pi_{\mathcal{F}}$. Since the distributions in $\mathcal{F}_{0}$ have compact supports, the distributions in $\mathcal{G}_{0}$, the support of $\Pi_{\mathcal{G}}$, should have the same support for (4.4) to hold. Hence, we only consider truncated normal distributions truncated on $\left[-M_{b}, M_{b}\right]^{q}$ with positive definite covariance matrixes. That is, $\mathcal{G}_{0}=\left\{N_{M_{b}}(0, \Sigma): 0<\rho_{1} \leq \rho_{\min }(\Sigma) \leq \rho_{\max }(\Sigma) \leq \rho_{2}<\infty\right\}$ for some constants $\rho_{1}$ and $\rho_{2}$, where $N_{M_{b}}(0, \Sigma)$ denotes the truncated normal distribution. Let $\Pi_{\mathcal{H}}=\Pi_{\mathcal{F}} \times \Pi_{\mathcal{G}}$.

Corollary 4.2. Assume that $\liminf _{n} \rho_{\min }\left(\mathbf{Z}_{n}\right)>0$ and $\rho_{\min }\left(v_{\eta_{0}}(w)\right)>0$ for every $w$. With the prior $\Pi_{\mathcal{H}}$ described above, the BvM theorem holds for the linear mixed regression model provided that $\eta_{0} \in \mathcal{H}_{0}$, and $\Pi_{\Theta}$ is compactly supported and thick at $\theta_{0}$ provided 4.1) and (4.3) hold.

Proof. Replacing $Q$ and $Q_{0}$ as constant functions, the proof is almost identical to that of Corollary 4.1, except for the proof of asymptotic tightness of 4.6), which is proved in Section A.2.8.

\section{$5 \quad$ Numerical study}

In this section, we provide simulation results to illustrate semi-parametric efficacy of the Bayes estimator in the linear mixed effect model. We specialize the model introduced in section 4 slightly: we only consider the random intercept model,

$$
X_{i j}=\theta^{T} Z_{i j}+b_{i}+\epsilon_{i j}
$$

where the $b_{i}$ 's are univariate random effects following a normal distribution with mean 0 and variance $\sigma_{b}^{2}$. In simulations, a dataset is generated from model (5.1) with various error distributions. Then, the regression parameters $\theta$ are estimated using various methods including both frequentist and Bayesian approaches for comparison. This procedure is 
Figure 1: Density plots of error distribution in E4 (left) and E5 (right).
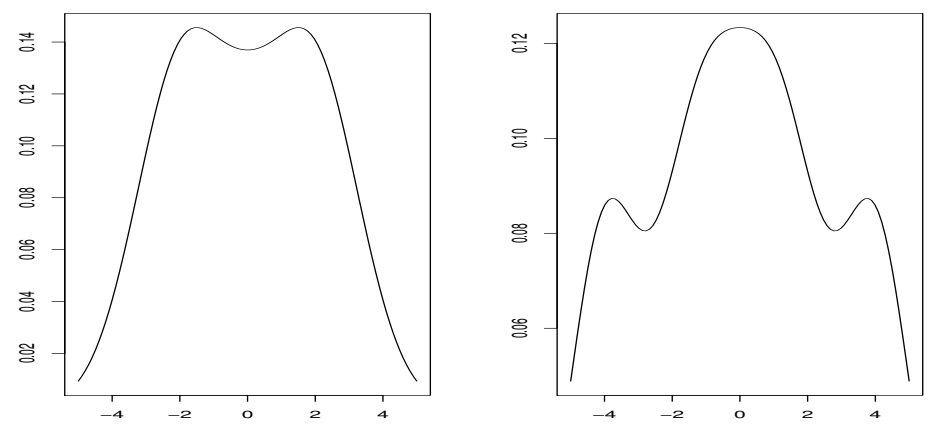

repeated $N$ times and the performance of estimation methods is evaluated by mean squared error, $N^{-1} \sum_{k=1}^{N}\left|\hat{\theta}_{n}^{(k)}-\theta_{0}\right|^{2}$, where $\hat{\theta}_{n}^{(k)}$ is the estimate in the $k$ th simulation. We compare the performance of 3 estimators under 5 error distributions. In all simulations we let $Z_{i j}=\left(Z_{i j 1}, Z_{i j 2}\right)^{T}$, where the $Z_{i j k}$ 's are generated i.i.d. from the Bernoulli distribution with success probability $1 / 2$. The true parameters $\theta_{0}$ and $\sigma_{0 b}^{2}$ are set to be $(-1,1)^{T}$ and 1 , respectively. For the error distribution, we consider the standard normal distribution (E1), the Student $t$-distributions with 2 degree of freedom (E2), the uniform(-3,3) distribution (E3), and two mixtures of normal distribution (E4 and E5). For the mixtures we take,

$$
p(x)=\sum_{k=1}^{K} \pi_{k}\left(\phi_{1}\left(x-\mu_{k}\right)+\phi_{1}\left(x+\mu_{k}\right)\right),
$$

with $K=4$,

$$
\left(\mu_{1}, \mu_{2}, \mu_{3}, \mu_{4}\right)=(0,1.5,2.5,3.5) ; \quad\left(\pi_{1}, \pi_{2}, \pi_{3}, \pi_{4}\right)=(0.1,0.2,0.15,0.05),
$$

for $\mathrm{E} 4$, and $K=4$,

$$
\left(\mu_{1}, \mu_{2}, \mu_{3}, \mu_{4}\right)=(0,1,2,4) ; \quad\left(\pi_{1}, \pi_{2}, \pi_{3}, \pi_{4}\right)=(0.05,0.15,0.1,0.2),
$$

for E5. These two densities (see Figure 1) have two and three modes, respectively.

For the estimators of $\theta$, we consider one frequentist estimator $(\mathrm{F})$ (the maximum likelihood estimator under the assumption of a normal error and normal random effect, which is equal to Henderson's best linear unbiased estimator [16]), and two Bayesian estimators (B1 and B2). For the two Bayes estimators, we consider two different priors for the distribution of $\eta$ : the normal distributions with mean 0 and variance $\sigma_{\epsilon}^{2}$ for $f$ and normal distribution with mean 0 and variance $\sigma_{b}^{2}$ for $G(\mathrm{~B} 1)$, and a symmetrized Dirichlet process mixture for $f$ and normal distribution with mean 0 and variance $\sigma_{b}^{2}$ for $G(\mathrm{~B} 2)$. Independent inverse Gamma distributions are used for the priors of $\sigma_{\epsilon}^{2}$ and $\sigma_{b}^{2}$, and independent diffuse normal distributions are used for the prior of $\theta$.

For each error distribution, $N=300$ datasets with $n=20$ and $m_{i}=5$ for all $i$, are generated. The mean squared errors and relative efficiencies (with respect to B2) of the 
Table 1: Mean squared error (and relative efficiency with respect to B2) of each methods F, B1 and B2 among $N=300$ repetitions for each experiment E1-E5.

\begin{tabular}{c|rrr}
\hline \multicolumn{2}{c}{$\mathrm{F}$} & $\mathrm{B} 1$ & $\mathrm{~B} 2$ \\
\hline E1 & 0.03 & 0.03 & 0.03 \\
& $(0.98)$ & $(0.98)$ & $(1.00)$ \\
E2 & 0.27 & 0.26 & 0.09 \\
& $(3.06)$ & $(2.99)$ & $(1.00)$ \\
E3 & 0.07 & 0.07 & 0.05 \\
& $(1.40)$ & $(1.39)$ & $(1.00)$ \\
E4 & 0.13 & 0.12 & 0.11 \\
& $(1.18)$ & $(1.16)$ & $(1.00)$ \\
E5 & 0.19 & 0.19 & 0.17 \\
& $(1.13)$ & $(1.12)$ & $(1.00)$ \\
\hline
\end{tabular}

three estimators are summarized in Table 1, B2 dominates the other two estimators when the error distribution is other than the normal. In particular, the losses of efficiency for $\mathrm{F}$ and B1 compared to B2 are relatively large when the error distribution has a heavier tail than the normal distribution (e.g. E2).

\section{A Appendix}

\section{A.1 Proofs for Section 3}

\section{A.1.1 Proof of 3.10}

Since,

$$
\begin{aligned}
& \sup _{\eta \in \mathcal{H}^{N}}\left|\frac{1}{n} P_{0}^{(n)}\left(\ell_{\theta, \eta}^{(n)}-\ell_{\theta_{0}, \eta}^{(n)}\right)+\frac{1}{2}\left(\theta-\theta_{0}\right)^{T} V_{n, \eta}\left(\theta-\theta_{0}\right)\right| \\
\leq & \sup _{i \geq 1} \sup _{\eta \in \mathcal{H}^{N}}\left|P_{\eta_{0}} \log \frac{\eta\left(X-\left(\theta-\theta_{0}\right)^{T} Z_{i}\right)}{\eta(X)}+\frac{1}{2} v_{\eta}\left(\theta-\theta_{0}\right)^{T} Z_{i} Z_{i}^{T}\left(\theta-\theta_{0}\right)\right|,
\end{aligned}
$$

where $X \sim P_{\eta_{0}}$, it suffices to show that,

$$
\sup _{\eta \in \mathcal{H}^{N}}\left|P_{\eta_{0}} \log \frac{\eta(X-y)}{\eta(X)}+\frac{y^{2}}{2} v_{\eta}\right|=o\left(y^{2}\right)
$$

as $y \rightarrow 0$.

We consider only the case $y>0$; the case $y<0$ is treated similarly. For $\eta \in \mathcal{H}^{N}$, we 
have,

$$
\begin{aligned}
& \int \log \frac{\eta(x-y)}{\eta(x)} \eta_{0}(x) d x \\
& =\int_{-\infty}^{0} \log \frac{\eta(x-y / 2)}{\eta(x+y / 2)} \eta_{0}(x+y / 2) d x+\int_{0}^{\infty} \log \frac{\eta(x-y / 2)}{\eta(x+y / 2)} \eta_{0}(x+y / 2) d x \\
& =\int_{0}^{\infty} \log \frac{\eta(-x-y / 2)}{\eta(-x+y / 2)} \eta_{0}(-x+y / 2) d x+\int_{0}^{\infty} \log \frac{\eta(x-y / 2)}{\eta(x+y / 2)} \eta_{0}(x+y / 2) d x \\
& =\int_{0}^{\infty} \log \frac{\eta(x+y / 2)}{\eta(x-y / 2)} \eta_{0}(x-y / 2) d x+\int_{0}^{\infty} \log \frac{\eta(x-y / 2)}{\eta(x+y / 2)} \eta_{0}(x+y / 2) d x \\
& =-\int_{0}^{\infty}\left[\ell_{\eta}\left(x-\frac{y}{2}\right)-\ell_{\eta}\left(x+\frac{y}{2}\right)\right] \cdot\left[\eta_{0}\left(x-\frac{y}{2}\right)-\eta_{0}\left(x+\frac{y}{2}\right)\right] d x \\
& =-\int_{-y / 2}^{\infty}\left[\ell_{\eta}(x+y)-\ell_{\eta}(x)\right] \cdot\left[\eta_{0}(x+y)-\eta_{0}(x)\right] d x \\
& =-\int_{0}^{\infty}\left[\ell_{\eta}(x+y)-\ell_{\eta}(x)\right] \cdot\left[\eta_{0}(x+y)-\eta_{0}(x)\right] d x+R(y, \eta)
\end{aligned}
$$

where the third equality holds by the symmetry of $\eta$ and $\eta_{0}$, and,

$$
R_{n}(y, \eta)=-\int_{-y / 2}^{0}\left[\ell_{\eta}(x+y)-\ell_{\eta}(x)\right] \cdot\left[\eta_{0}(x+y)-\eta_{0}(x)\right] d x .
$$

Note that $\sup _{\eta \in \mathcal{H}^{N}}|R(y, \eta)|=o\left(y^{2}\right)$ as $y \rightarrow 0$ because 3.6 implies,

$$
\begin{aligned}
|R(y, \eta)| & =\left|\int_{-y / 2}^{0}\left[\ell_{\eta}(x+y)-\ell_{\eta}(x)\right] \cdot\left[\eta_{0}(x+y)-\eta_{0}(x)\right] d x\right| \\
& =y \cdot\left|\int_{-y / 2}^{0} \int_{0}^{1}\left[\ell_{\eta}(x+y)-\ell_{\eta}(x)\right] \cdot \dot{\eta}_{0}(x+t y) d t d x\right| \\
& \leq 2 y^{2} \int_{0}^{1} \int_{-y / 2}^{0} Q(x+t y) \cdot\left|s_{\eta_{0}}(x+t y)\right| \cdot \eta_{0}(x+t y) d x d t \\
& \leq 2 y^{2} \int_{0}^{1} \int_{-y / 2}^{0} Q^{2}(x+t y) \cdot \eta_{0}(x+t y) d x d t \lesssim y^{3}
\end{aligned}
$$

for small enough $y$ by the continuity of $Q$ and $\eta_{0}$, where $\dot{\eta}(x)=\partial \eta(x) / \partial x$. Finally, a Taylor expansion and Fubini's theorem imply that the last integral of $(A .2)$ is equal to,

$$
y^{2} \int_{0}^{1} \int_{0}^{1} \int_{0}^{\infty} s_{\eta}(x+t y) \dot{\eta}_{0}(x+s y) d x d t d s
$$

Since,

$$
\frac{y^{2}}{2} v_{\eta}=-y^{2} \int_{0}^{\infty} s_{\eta}(x) \dot{\eta}_{0}(x) d x
$$


the sum of A.3 and A.4 is bounded by,

$$
\begin{aligned}
& y^{2}\left|\int_{0}^{1} \int_{0}^{1} \int_{0}^{\infty} s_{\eta}(x+t y) \dot{\eta}_{0}(x+s y)-s_{\eta}(x) \dot{\eta}_{0}(x) d x d t d s\right| \\
& \leq y^{2} \int_{0}^{1} \int_{0}^{1} \int_{0}^{\infty}\left|\left\{s_{\eta}(x+t y)-s_{\eta}(x+s y)\right\} \dot{\eta}_{0}(x+s y)\right| d x d t d s \\
& \quad+y^{2}\left|\int_{0}^{1} \int_{0}^{\infty}\left[s_{\eta}(x+s y) \dot{\eta}_{0}(x+s y)-s_{\eta}(x) \dot{\eta}_{0}(x)\right] d x d s\right| \\
& \leq y^{3} \int Q(x)\left|\dot{\eta}_{0}(x)\right| d x+y^{2} \sup _{s \in[0,1]}\left|\int_{0}^{s y} s_{\eta}(x) \dot{\eta}_{0}(x) d x\right| \\
& \leq y^{3} P_{\eta_{0}} Q^{2}+y^{2} \int_{0}^{y} Q^{2}(x) \eta_{0}(x) d x=O\left(y^{3}\right),
\end{aligned}
$$

as $y \rightarrow 0$.

\section{A.1.2 Proof of $(3.12)$}

For a sequence $\left(\eta_{n}\right)$ such that $\eta_{n} \in \mathcal{H}_{n}$ and $\sup _{\eta \in \mathcal{H}_{n}} d_{2}\left(\eta, \eta_{0}\right)<d_{2}\left(\eta_{n}, \eta_{0}\right)+n^{-1}$, it suffices to show that $d_{2}\left(\eta_{n}, \eta_{0}\right) \rightarrow 0$. By the definition of $\mathcal{H}_{n}$, we have $h\left(\eta_{n}, \eta_{0}\right) \rightarrow 0$. We first prove that $\ell_{\eta_{n}}$ converges to $\ell_{\eta_{0}}$ pointwise. Suppose $\ell_{\eta_{n}}(x) \nrightarrow \ell_{\eta_{0}}(x)$ for some $x \in \mathbb{D}$. Then we can choose an $\epsilon>0$ and a subsequence $m(n)$ such that $m(n) \geq N$ and $\left|\ell_{\eta_{m(n)}}(x)-\ell_{\eta_{0}}(x)\right|>\epsilon$ for every $n$. Note that $\ell_{\eta}$ is continuously differentiable and the derivative of $\ell_{\eta}$ is bounded by a continuous function $Q$ uniformly in $\eta \in \mathcal{H}^{N}$ by (3.6). Thus we can choose a $\delta>0$ such that $\left|\ell_{\eta_{m(n)}}(y)-\ell_{\eta_{0}}(y)\right|>\epsilon / 2$ for every $n \geq 1$ and $y$ with $|y-x|<\delta$. Note that $\delta>0$ can be chosen sufficiently small so that $\eta_{0}(y)>\eta_{0}(x) / 2$ for every $y$ with $|y-x|<\delta$. Since $\ell_{\eta_{m(n)}}(y)-\ell_{\eta_{0}}(y)=2 \log \sqrt{\eta_{m(n)}(y) / \eta_{0}(y)}$, there exists a $\bar{\epsilon}>0$ such that $\left|1-\sqrt{\eta_{m(n)}(y) / \eta_{0}(y)}\right|>\bar{\epsilon}$ for every $n \geq 1$ and $y$ with $|y-x|<\delta$. Note that,

$$
h^{2}\left(\eta_{m(n)}, \eta_{0}\right) \geq \int_{(x-\delta, x+\delta)}\left(1-\sqrt{\frac{\eta_{m(n)}}{\eta_{0}}}\right)^{2} d P_{\eta_{0}} \geq \delta \bar{\epsilon}^{2} \eta_{0}(x)>0,
$$

for every $n \geq 1$, which contradicts $h\left(\eta_{n}, \eta_{0}\right) \rightarrow 0$. Conclude that $\ell_{\eta_{n}}(x) \rightarrow \ell_{\eta_{0}}(x)$, for every $x$.

By (3.6), we have for every sufficiently small $y>0$,

$$
\begin{aligned}
& \sup _{\eta \in \mathcal{H}^{N}} \mid \int {\left[\frac{\ell_{\eta}(x+y)-\ell_{\eta}(x)}{y}+s_{\eta_{0}}(x)\right]^{2}-\left(s_{\eta}(x)-s_{\eta_{0}}(x)\right)^{2} d P_{\eta_{0}}(x) \mid } \\
&=\sup _{\eta \in \mathcal{H}^{N}} \mid \int\left\{\int_{0}^{1}\left[s_{\eta}(x+t y)-s_{\eta}(x)\right] d t\right. \\
&\left.\times\left[\frac{\ell_{\eta}(x+y)-\ell_{\eta}(x)}{y}-s_{\eta}(x)+2 s_{\eta_{0}}(x)\right]\right\} d P_{\eta_{0}}(x) \mid \\
& \leq \sup _{\eta \in \mathcal{H}^{N}} y\left|\int Q(x) \times\left[\frac{\ell_{\eta}(x+y)-\ell_{\eta}(x)}{y}-s_{\eta}(x)+2 s_{\eta_{0}}(x)\right] d P_{\eta_{0}}(x)\right| \\
&=o(1),
\end{aligned}
$$


as $y \downarrow 0$. By the Moore-Osgood theorem [32], this enables us to interchange the two limits in the following equality

$$
\begin{aligned}
\lim _{n \rightarrow \infty} P_{\eta_{0}}\left(s_{\eta_{n}}-s_{\eta_{0}}\right)^{2} & =\lim _{n \rightarrow \infty} \lim _{y \downarrow 0} \int\left[\frac{\ell_{\eta_{n}}(x+y)-\ell_{\eta_{n}}(x)}{y}+s_{\eta_{0}}(x)\right]^{2} d P_{\eta_{0}}(x) \\
& =\lim _{y \downarrow 0} \lim _{n \rightarrow \infty} \int\left[\frac{\ell_{\eta_{n}}(x+y)-\ell_{\eta_{n}}(x)}{y}+s_{\eta_{0}}(x)\right]^{2} d P_{\eta_{0}}(x) .
\end{aligned}
$$

The right-hand side of A.5 is equal to 0 by dominated convergence based on pointwise convergence of $\ell_{\eta_{n}}$ to $\ell_{\eta_{0}}$.

\section{A.1.3 Proof of Lemma 3.1}

Since $\eta_{0}$ is continuous and $\eta_{0}(0)>0$, there exist constants $C>0$ and $\delta>0$ such that $\int_{\gamma}^{\infty} \eta_{0}(x) d x<1 / 2-C(\gamma \wedge \delta)$ for every $\gamma>0$. Let $\epsilon>0$ be a constant such that $\epsilon<a C \delta$, where $a^{2}=\liminf _{n} \rho_{\min }\left(\mathbf{Z}_{n}\right) /\left(2 L^{2}\right)$.

For a given large enough $n$, fix $\eta \in \mathcal{H}_{n}$ with $h_{n}\left((\theta, \eta),\left(\theta_{0}, \eta_{0}\right)\right)<\epsilon$. Since the Hellinger distance is bounded below by half of the total variational distance, we have,

$$
h^{2}\left(p_{\theta, \eta, i}, p_{\theta_{0}, \eta_{0}, i}\right) \geq d_{V}^{2}\left(p_{\theta, \eta, i}, p_{\theta_{0}, \eta_{0}, i}\right) / 4=\sup _{B}\left|P_{\theta, \eta, i}(B)-P_{\theta_{0}, \eta_{0}, i}(B)\right|^{2} .
$$

By letting $B=\left[\theta^{T} Z_{i}, \infty\right)$ in A.6, we have,

$$
h^{2}\left(p_{\theta, \eta, i}, p_{\theta_{0}, \eta_{0}, i}\right) \geq\left(\int_{\left|\left(\theta-\theta_{0}\right)^{T} Z_{i}\right|}^{\infty} \eta_{0}(x) d x-\frac{1}{2}\right)^{2} \geq C^{2}\left(\left|\left(\theta-\theta_{0}\right)^{T} Z_{i}\right| \wedge \delta\right)^{2} .
$$

Let $\mathbb{N}_{\delta, n}=\left\{i \leq n:\left|\left(\theta-\theta_{0}\right)^{T} Z_{i}\right| \geq \delta\right\}$ and let $N_{\delta, n}$ denote its cardinality. Then A.7 implies,

$$
\begin{aligned}
\left.\epsilon^{2} \geq h_{n}^{2}\left((\theta, \eta), \theta_{0}, \eta_{0}\right)\right) & \geq \frac{C^{2}}{n} \sum_{i=1}^{n}\left(\left|\left(\theta-\theta_{0}\right)^{T} Z_{i}\right| \wedge \delta\right)^{2} \\
& \geq \frac{C^{2} N_{\delta, n} \delta^{2}}{n}+\frac{C^{2}}{n} \sum_{i \notin \mathbb{N}_{\delta, n}}\left|\left(\theta-\theta_{0}\right)^{T} Z_{i}\right|^{2}
\end{aligned}
$$

The first term of A.8 is greater than $N_{\delta, n} \epsilon^{2} /\left(n a^{2}\right)$ since $\epsilon<a C \delta$, which implies $N_{\delta, n} / n<$ $a^{2}$. On the other hand, for the second term of A.8, note that,

$$
\sum_{i \notin \mathbb{N}_{\delta, n}}\left|\left(\theta-\theta_{0}\right)^{T} Z_{i}\right|^{2} \geq \sum_{i=1}^{n}\left|\left(\theta-\theta_{0}\right)^{T} Z_{i}\right|^{2}-N_{\delta, n} \max _{i}\left|\left(\theta-\theta_{0}\right)^{T} Z_{i}\right|^{2}
$$

Since $\sum_{i=1}^{n}\left|\left(\theta-\theta_{0}\right)^{T} Z_{i}\right|^{2} \geq n\left|\theta-\theta_{0}\right|^{2} \rho_{\min }\left(\mathbf{Z}_{n}\right)$ and $\max _{i}\left|\left(\theta-\theta_{0}\right)^{T} Z_{i}\right|^{2} \leq L^{2}\left|\theta-\theta_{0}\right|^{2}$, we have,

$$
\frac{C^{2}}{n} \sum_{i \notin \mathbb{N}_{\delta, n}}\left|\left(\theta-\theta_{0}\right)^{T} Z_{i}\right|^{2} \geq C^{2}\left|\theta-\theta_{0}\right|^{2}\left(\rho_{\min }\left(\mathbf{Z}_{n}\right)-L^{2} \frac{N_{\delta, n}}{n}\right) .
$$

Since $N_{\delta, n} / n<a^{2}$ and $a^{2}=\liminf _{n} \rho_{\min }\left(\mathbf{Z}_{n}\right) /\left(2 L^{2}\right)$, A.8 and A.9 together imply $\left|\theta-\theta_{0}\right|^{2} \leq K_{1} \epsilon^{2}$, where $K_{1}=2 /\left(C^{2} \rho_{\min }\left(\mathbf{Z}_{n}\right)\right)$. 
The proof is complete if we show that $h\left(\eta, \eta_{0}\right)<K \epsilon$ for some constant $K>0$. Note that for every $i$,

$$
h^{2}\left(\eta, \eta_{0}\right)=h^{2}\left(p_{\theta, \eta, i}, p_{\theta, \eta_{0}, i}\right) \leq 2\left(h^{2}\left(p_{\theta, \eta, i}, p_{\theta_{0}, \eta_{0}, i}\right)+h^{2}\left(p_{\theta_{0}, \eta_{0}, i}, p_{\theta, \eta_{0}, i}\right)\right) .
$$

In addition, there exists a constant $K_{2}>0$ such that,

$$
\sup _{i} h^{2}\left(p_{\theta_{0}, \eta_{0}, i}, p_{\theta, \eta_{0}, i}\right) \leq K_{2}\left|\theta-\theta_{0}\right|^{2},
$$

for every $\theta$ that is sufficiently close to $\theta_{0}$ because (denote $\dot{\eta}_{0}=d \eta_{0} / d x$ ),

$$
\begin{aligned}
& \int\left(\sqrt{\eta_{0}(x+y)}-\sqrt{\eta_{0}(x)}\right)^{2} d x=y^{2} \int\left(\int_{0}^{1} \frac{\dot{\eta}_{0}(x+t y)}{\sqrt{\eta_{0}(x+t y)}} d t\right)^{2} d x \\
& \leq y^{2} \iint_{0}^{1}\left(\frac{\dot{\eta}_{0}(x+t y)}{\eta_{0}(x+t y)}\right)^{2} \eta_{0}(x+t y) d t d x \leq y^{2} P_{\eta_{0}} Q^{2}
\end{aligned}
$$

for small enough $y$, where the last inequality holds by Fubini's theorem and (3.6). So we have,

$$
\begin{aligned}
h^{2}\left(\eta, \eta_{0}\right) & \leq \frac{1}{n} \sum_{i=1}^{n} 2\left(h^{2}\left(p_{\theta, \eta, i}, p_{\theta_{0}, \eta_{0}, i}\right)+h^{2}\left(p_{\theta_{0}, \eta_{0}, i}, p_{\theta, \eta_{0}, i}\right)\right) \\
& \leq 2 h_{n}^{2}\left((\theta, \eta),\left(\theta_{0}, \eta_{0}\right)\right)+2 K_{2}\left|\theta-\theta_{0}\right|^{2}
\end{aligned}
$$

where the first inequality holds by A.10) and the second inequality holds by the definition of $h_{n}$ and (A.11). Since we have already shown that $\left|\theta-\theta_{0}\right|^{2}<K_{1} \epsilon^{2}$, we conclude that $h\left(\eta, \eta_{0}\right) \leq K \epsilon$, where $K=\sqrt{2+2 K_{1} K_{2}}$.

\section{A.1.4 Proof of $(3.16)$}

We start by proving the following two claims: for every $\widetilde{M}_{n} \rightarrow \infty$ with $\widetilde{M}_{n} / \sqrt{n} \rightarrow 0$,

$$
\sup _{|h| \leq \widetilde{M}_{n}} \sup _{\eta \in \mathcal{H}^{N}}\left|\left(\ell_{\theta_{n}(h), \eta}^{(n)}-\ell_{\theta_{0}, \eta}^{(n)}-\frac{h^{T}}{\sqrt{n}} \dot{\ell}_{\theta_{0}, \eta}^{(n)}\right)^{o}\right|=o_{P_{0}}\left(\widetilde{M}_{n}^{2}\right),
$$

and,

$$
\sup _{\widetilde{M}_{n}<|h|<\epsilon \sqrt{n}} \sup _{\eta \in \mathcal{H}^{N}}\left|\left(\ell_{\theta_{n}(h), \eta}^{(n)}-\ell_{\theta_{0}, \eta}^{(n)}\right)^{o}\right| \cdot|h|^{-2}=o_{P_{0}}(1),
$$

for sufficiently small $\epsilon>0$.

First, the equality,

$$
\left(\ell_{\theta_{n}(h), \eta}^{(n)}-\ell_{\theta_{0}, \eta}^{(n)}-\frac{h^{T}}{\sqrt{n}} \dot{\ell}_{\theta_{0}, \eta}^{(n)}\right)^{o}=\frac{h^{T}}{\sqrt{n}} \int_{0}^{1}\left(\dot{\ell}_{\theta_{n}(t h), \eta}^{(n)}-\dot{\ell}_{\theta_{0}, \eta}^{(n)}\right)^{o} d t
$$

implies that the left-hand side of $\mathrm{A} .12$ is bounded by,

$$
\sup _{|h| \leq \widetilde{M}_{n}} \sup _{\eta \in \mathcal{H}^{N}}\left|\frac{\widetilde{M}_{n}}{\sqrt{n}}\left(\dot{\ell}_{\theta_{n}(h), \eta}^{(n)}-\dot{\ell}_{\theta_{0}, \eta}^{(n)}\right)^{o}\right| .
$$


Since,

$$
\sup _{|h| \leq \widetilde{M}_{n}} \sup _{\eta \in \mathcal{H}^{N}}\left|\frac{1}{\sqrt{n}}\left(\dot{\ell}_{\theta_{n}(h), \eta}^{(n)}-\dot{\ell}_{\theta_{0}, \eta}^{(n)}\right)^{o}\right|=O_{P_{0}}(1),
$$

by asymptotic tightness of (3.7), we conclude A.14 is of order $o_{P_{0}}\left(\widetilde{M}_{n}^{2}\right)$.

Similarly by the equality,

$$
\left(\ell_{\theta_{n}(h), \eta}^{(n)}-\ell_{\theta_{0}, \eta}^{(n)}\right)^{o}=\frac{h^{T}}{\sqrt{n}} \int_{0}^{1}\left(\dot{\ell}_{\theta_{n}(t h), \eta}^{(n)}\right)^{o} d t
$$

the left-hand side of A.13 is bounded by,

$$
\sup _{\widetilde{M}_{n}<|h|<\epsilon \sqrt{n}} \sup _{\eta \in \mathcal{H}_{n}}\left|\frac{h^{T}}{\sqrt{n}}\left(\dot{\ell}_{\theta_{n}(h), \eta}^{(n)}\right)^{o}\right| \cdot|h|^{-2} .
$$

By asymptotic tightness of (3.7),

$$
\sup _{\widetilde{M}_{n}<|h|<\epsilon \sqrt{n}} \sup _{\eta \in \mathcal{H}_{n}}\left|\frac{1}{\sqrt{n}}\left(\dot{\ell}_{\theta_{n}(h), \eta}^{(n)}\right)^{o}\right|=O_{P_{0}}(1),
$$

so A.15 is of order $o_{P_{0}}(1)$.

Next, we show that for every $C_{1}>0$, there exists a $C_{2}>0$ such that,

$$
P_{0}^{(n)}\left(\left\{\inf _{\eta \in \mathcal{H}_{n}} \int_{\Theta} \frac{p_{\theta, \eta}^{(n)}}{p_{\theta_{0}, \eta}^{(n)}} d \Pi_{\Theta}(\theta) \geq C_{2}\left(\frac{M_{n}}{\sqrt{n}}\right)^{p} e^{-C_{1} M_{n}^{2}}\right\}\right) \rightarrow 1 .
$$

Let,

$$
\Phi_{n}(h, \eta)=\ell_{\theta_{n}(h), \eta}^{(n)}-\ell_{\theta_{0}, \eta}^{(n)}=\sum_{i=1}^{5} A_{n, i}(h, \eta),
$$

where,

$$
\begin{aligned}
A_{n, 1}(h, \eta) & =\left(\ell_{\theta_{n}(h), \eta}^{(n)}-\ell_{\theta_{0}, \eta}^{(n)}-\frac{h^{T}}{\sqrt{n}} \dot{\ell}_{\theta_{0}, \eta}^{(n)}\right)^{o}, \\
A_{n, 2}(h, \eta) & =\frac{1}{2} h^{T}\left(V_{n, \eta_{0}}-V_{n, \eta}\right) h, \\
A_{n, 3}(h, \eta) & =\frac{h^{T}}{\sqrt{n}}\left(\dot{\ell}_{\theta_{0}, \eta}^{(n)}-P_{0}^{(n)} \dot{\ell}_{\theta_{0}, \eta}^{(n)}\right), \\
A_{n, 4}(h, \eta) & =-\frac{1}{2} h^{T} V_{n, \eta_{0}} h, \\
A_{n, 5}(h, \eta) & =P_{0}^{(n)}\left(\ell_{\theta_{n}(h), \eta}^{(n)}-\ell_{\theta_{0}, \eta}^{(n)}\right)+\frac{1}{2} h^{T} V_{n, \eta} h .
\end{aligned}
$$

Note that $\int \exp \left(\Phi_{n}(h, \eta)\right) d \Pi_{n}(h) \geq \int_{|h| \leq C_{1} M_{n}} \exp \left(\Phi_{n}(h, \eta)\right) d \Pi_{n}(h)$, where $\Pi_{n}$ is the prior for the centred and rescaled parameter $h=\sqrt{n}\left(\theta-\theta_{0}\right)$. For $h$ and $\eta \in \mathcal{H}_{n}$ with $|h| \leq$ $C_{1} M_{n}$, the suprema of $\left|A_{n, 1}(h, \eta)\right|$ and $\left|A_{n, 2}(h, \eta)\right|$ are of order $o_{P_{0}}\left(M_{n}^{2}\right)$ by A.12 and 2.3. respectively. The supremum of $\left|A_{n, 3}(h, \eta)\right|$ is of the same order by asymptotic tightness of (3.7). The quantity $\left|A_{n, 4}(h, \eta)\right|$ is uniformly bounded by $C_{1}^{2} M_{n}^{2}\left\|V_{n, \eta_{0}}\right\| / 2$ and 
the supremum of $\left|A_{n, 5}(h, \eta)\right|$ is of order $o\left(M_{n}^{2}\right)$ by (3.10). Therefore, for $|h| \leq C_{1} M_{n}$ and $\eta \in \mathcal{H}_{n}, \Phi_{n}(h, \eta)$ is uniformly bounded below by,

$$
M_{n}^{2}\left(-\frac{C_{1}^{2}}{2} \cdot\left\|V_{n, \eta_{0}}\right\|+o_{P_{0}}(1)\right) .
$$

Thus,

$$
\begin{aligned}
\int_{\Theta} \frac{p_{\theta, \eta}^{(n)}}{p_{\theta_{0}, \eta}^{(n)}} d \Pi_{\Theta}(\theta) \geq \int_{|h| \leq C_{1} M_{n}} \exp \left(\Phi_{n}(h, \eta)\right) d \Pi_{n}(h) \\
\quad \geq \int_{\sqrt{n}\left|\theta-\theta_{0}\right| \leq C_{1} M_{n}} \exp \left[M_{n}^{2}\left(-\frac{C_{1}^{2}}{2} \cdot\left\|V_{n, \eta_{0}}\right\|+o_{P_{0}}(1)\right)\right] d \Pi_{\Theta}(\theta) .
\end{aligned}
$$

Also, the thickness of $\Pi_{\Theta}$ at $\theta_{0}$ implies that,

$$
\Pi_{\Theta}\left\{\theta: \sqrt{n}\left|\theta-\theta_{0}\right| \leq C_{1} M_{n}\right\} \geq C_{2}\left(M_{n} / \sqrt{n}\right)^{p},
$$

for some $C_{2}>0$. Since $\lim \sup _{n} \rho_{\max }\left(V_{n, \eta_{0}}\right)<\infty$ by (2.4), and $C_{1}>0$ is arbitrary, we conclude that A.16 holds.

Finally, we prove that there exist $C>0$ and $\epsilon>0$ such that,

$$
P_{0}^{(n)}\left(\sup _{M_{n}<|h|<\epsilon \sqrt{n}} \sup _{\eta \in \mathcal{H}_{n}} \frac{p_{\theta_{n}(h), \eta}^{(n)}}{p_{\theta_{0}, \eta}^{(n)}} e^{C|h|^{2}} \leq 1\right) \rightarrow 1 .
$$

For given $\delta>0$, by (3.10), there exists an $\epsilon>0$ such that

$$
\sup _{\eta \in \mathcal{H}^{N}}\left|P_{0}^{(n)}\left(\ell_{\theta_{n}(h), \eta}^{(n)}-\ell_{\theta_{0}, \eta}^{(n)}\right)+\frac{1}{2} h^{T} V_{n, \eta} h\right|<\delta \cdot|h|^{2},
$$

for every $h$ with $|h|<\sqrt{n} \epsilon$. Write,

$$
\log \frac{p_{\theta_{n}(h), \eta}^{(n)}}{p_{\theta_{0}, \eta}^{(n)}}=\sum_{i=1}^{4} B_{n, i}(h, \eta)
$$

where,

$$
\begin{aligned}
B_{n, 1}(h, \eta) & =\left(\ell_{\theta_{n}(h), \eta}^{(n)}-\ell_{\theta_{0}, \eta}^{(n)}\right)^{o}, \\
B_{n, 2}(h, \eta) & =P_{0}^{(n)}\left(\ell_{\theta_{n}(h), \eta}^{(n)}-\ell_{\theta_{0}, \eta}^{(n)}\right)+\frac{1}{2} h^{T} V_{n, \eta} h, \\
B_{n, 3}(h, \eta) & =\frac{1}{2} h^{T}\left(V_{n, \eta_{0}}-V_{n, \eta}\right) h, \\
B_{n, 4}(h, \eta) & =-\frac{1}{2} h^{T} V_{n, \eta_{0}} h .
\end{aligned}
$$

For $M_{n}<|h|<\epsilon \sqrt{n}$ and $\eta \in \mathcal{H}_{n},\left|B_{n, 1}(h, \eta)\right|$ and $\left|B_{n, 3}(h, \eta)\right|$ are bounded by $|h|^{2} \times o_{P_{0}}(1)$ by A.13) and (2.3), respectively, where the $o_{P_{0}}(1)$ term does not depend on $h$ and $\eta$. Furthermore, $\left|B_{n, 2}(h, \eta)\right| \leq \delta|h|^{2}$ by A.19), and $B_{n, 4}(h, \eta) \leq-\rho_{\min }\left(V_{n, \eta_{0}}\right)|h|^{2} / 2$. Thus, A.20 is bounded above by,

$$
|h|^{2} \cdot\left(-\frac{1}{2} \rho_{\min }\left(V_{n, \eta_{0}}\right)+\delta+o_{P_{0}}(1)\right),
$$

for every $h$ with $|h|<\sqrt{n} \epsilon$ and $\eta \in \mathcal{H}_{n}$. Since $\delta>0$ can be arbitrarily small and $\liminf _{n} \rho_{\min }\left(V_{n, \eta_{0}}\right)>0$ by (2.4), we conclude that A.18) holds for $C<\liminf _{n} \rho_{\min }\left(V_{n, \eta_{0}}\right) / 2$. 


\section{A.1.5 Proof of $(3.21)$}

For the first inequality of (3.21), note that,

$$
\left|\sqrt{a_{1}+a_{2}}-\sqrt{b_{1}+b_{2}}\right| \leq\left|\sqrt{a_{1}}-\sqrt{b_{1}}\right|+\left|\sqrt{a_{2}}-\sqrt{b_{2}}\right|
$$

and $\left(a_{1}+b_{1}\right)^{2} \leq 2\left(a_{1}^{2}+b_{1}^{2}\right)$ for any $a_{1}, a_{2}, b_{1}, b_{2} \geq 0$. Thus,

$$
\begin{aligned}
h^{2}(\bar{p}, \bar{q}) & =\int\left(\sqrt{\frac{p+p^{-}}{2}}-\sqrt{\frac{q+q^{-}}{2}}\right)^{2} d \mu \\
& \leq \int(\sqrt{p}-\sqrt{q})^{2}+\left(\sqrt{p^{-}}-\sqrt{q^{-}}\right)^{2} d \mu=2 h^{2}(p, q),
\end{aligned}
$$

and so $h(\bar{p}, \bar{q}) \leq \sqrt{2} h(p, q)$ for any two densities $p$ and $q$ supported on $\mathbb{D}$.

For the second and third inequalities of (3.21), we may assume that $p$ is symmetric. Then,

$$
\begin{aligned}
K(\bar{p}, \bar{q}) & =K(p, \bar{q})=\int\left(\log p-\log \left(\frac{q+q^{-}}{2}\right)\right) d P \\
& \leq \int\left(\log p-\frac{1}{2}\left\{\log \frac{q}{2}+\log \frac{q^{-}}{2}\right\}\right) d P=\int \frac{1}{2}\left(\log \frac{p}{q}+\log \frac{p}{q^{-}}\right) d P
\end{aligned}
$$

where the inequality holds by the concavity of $x \mapsto \log (x)$. Also, the symmetry of $p$ implies that $\int \log \left(p / q^{-}\right) d P=\int \log (p / q) d P$ and so $K(\bar{p}, \bar{q}) \leq K(p, q)$. In addition,

$$
V(\bar{p}, \bar{q})=V(p, \bar{q}) \leq \int\left(\log p-\log \left(\frac{q+q^{-}}{2}\right)\right)^{2} d P=\int\left(\log \frac{2 p}{q+q^{-}}\right)^{2} d P .
$$

Since $p / q \wedge p / q^{-} \leq 2 p /\left(q+q^{-}\right) \leq p / q \vee p / q^{-}$, we have,

$$
\left|\log \frac{2 p}{q+q^{-}}\right| \leq\left|\log \frac{p}{q}\right|+\left|\log \frac{p}{q^{-}}\right|
$$

and so $V(\bar{p}, \bar{q}) \leq 4 \int(\log (p / q))^{2} d P=4\left(V(p, q)+K^{2}(p, q)\right)$.

\section{A.1.6 Proof of $(3.22)$}

Assume that $\epsilon>0$ is sufficiently small and $\left|\theta_{1}-\theta_{2}\right| \vee\left|\theta-\theta_{0}\right|<\epsilon$. Using (3.19) and the fact that $(a+b)^{2} \leq 2\left(a^{2}+b^{2}\right)$ for all $a, b \in \mathbb{R}$, we have the second and third inequalities of $(3.22)$ because,

$$
\begin{aligned}
K\left(p_{\theta_{0}, \eta_{0}, i}, p_{\theta, \eta, i}\right) & =\int\left(\ell_{\theta_{0}, \eta_{0}, i}-\ell_{\theta_{0}, \eta, i}\right)+\left(\ell_{\theta_{0}, \eta, i}-\ell_{\theta, \eta, i}\right) d P_{\theta_{0}, \eta_{0}, i} \\
& \lesssim K\left(\eta_{0}, \eta\right)+\left|\theta-\theta_{0}\right|,
\end{aligned}
$$

and,

$$
\begin{aligned}
V\left(p_{\theta_{0}, \eta_{0}, i}, p_{\theta, \eta, i}\right) & \leq 2 \int\left(\ell_{\theta_{0}, \eta_{0}, i}-\ell_{\theta_{0}, \eta, i}\right)^{2}+\left(\ell_{\theta_{0}, \eta, i}-\ell_{\theta, \eta, i}\right)^{2} d P_{\theta_{0}, \eta_{0}, i} \\
& \lesssim V\left(\eta_{0}, \eta\right)+K^{2}\left(\eta_{0}, \eta\right)+\left|\theta-\theta_{0}\right|^{2},
\end{aligned}
$$


for every $\eta \in \mathcal{H}_{0}$.

For the first inequality of 3.22 ,

$$
\begin{array}{r}
h\left(p_{\theta_{1}, \eta_{1}, i}, p_{\theta_{2}, \eta_{2}, i}\right) \leq h\left(p_{\theta_{1}, \eta_{1}, i}, p_{\theta_{1}, \eta_{2}, i}\right)+h\left(p_{\theta_{1}, \eta_{2}, i}, p_{\theta_{2}, \eta_{2}, i}\right) \\
\quad=h\left(\eta_{1}, \eta_{2}\right)+h\left(p_{\theta_{1}, \eta_{2}, i}, p_{\theta_{2}, \eta_{2}, i}\right) \lesssim h\left(\eta_{1}, \eta_{2}\right)+\left|\theta_{1}-\theta_{2}\right|,
\end{array}
$$

for every $\eta_{1}, \eta_{2} \in \mathcal{H}_{0}$, where the last inequality holds because, with $\dot{\eta}(x)=d \eta(x) / d x$ and $y_{i}=\left|\left(\theta_{1}-\theta_{2}\right)^{T} Z_{i}\right|$,

$$
\begin{aligned}
h^{2}\left(p_{\theta_{1}, \eta, i}, p_{\theta_{2}, \eta, i}\right) & =\int\left(\sqrt{\eta\left(x+y_{i}\right)}-\sqrt{\eta(x)}\right)^{2} d x \\
& =y_{i}^{2} \int\left(\int_{0}^{1} \frac{\dot{\eta}\left(x+t y_{i}\right)}{\sqrt{\eta\left(x+t y_{i}\right)}} d t\right)^{2} d x \\
& \leq y_{i}^{2} \iint_{0}^{1}\left(\frac{\dot{\eta}\left(x+t y_{i}\right)}{\eta\left(x+t y_{i}\right)}\right)^{2} \eta\left(x+t y_{i}\right) d t d x \leq y_{i}^{2} P_{\eta} \widetilde{Q}^{2},
\end{aligned}
$$

for every $\eta \in \mathcal{H}_{0}$.

\section{A.1.7 Proof of the asymptotic tightness of $(3.23)$}

Without loss of generality we may assume that $\theta_{0}$ is equal to the zero vector. For given $a \in \mathbb{R}^{p}$, let,

$$
Z_{n i}(\theta, \eta)=a^{T} \dot{\ell}_{\theta, \eta, i} / \sqrt{n}, \quad S_{n i}=\sup _{\theta \in B_{\epsilon}} \sup _{\eta \in \mathcal{H}_{0}}\left|Z_{n i}(\theta, \eta)\right|,
$$

and $\mathcal{F}=B_{\epsilon} \times \mathcal{H}_{0}$. Let $N_{[]}^{n}(\delta, \mathcal{F})$ be the minimal number of sets $N$ in a partition $\left\{\mathcal{F}_{j}: 1 \leq\right.$ $j \leq N\}$ of $\mathcal{F}$ such that,

$$
\sum_{i=1}^{n} P_{0} \sup _{\substack{\left(\theta_{1}, \eta_{1}\right) \in \mathcal{F}_{j} \\\left(\theta_{2}, \eta_{2}\right) \in \mathcal{F}_{j}}}\left|Z_{n i}\left(\theta_{1}, \eta_{1}\right)-Z_{n i}\left(\theta_{2}, \eta_{2}\right)\right|^{2} \leq \delta^{2},
$$

for every $j \leq N$. The bracketing central limit theorem (Theorem 2.11.9 of [33]) assures that if ,

$$
\begin{aligned}
& \sum_{i=1}^{n} P_{0}\left(S_{n i} 1_{\left\{S_{n i}>\gamma\right\}}\right)=o(1) \quad \text { for every } \gamma>0, \\
& \int_{0}^{\delta_{n}} \sqrt{\log N_{[]}^{n}(\delta, \mathcal{F})} d \delta<\infty, \quad \text { for every } \delta_{n} \downarrow 0,
\end{aligned}
$$

then 3.23 is asymptotically tight.

Since $\left|Z_{i}\right|$ 's are bounded and the mean probability $H$ of the Dirichlet process is compactly supported, there exist functions $Q_{j}$ for $j=1,2$, such that $Q_{j}(x)=C_{j}\left(1+|x|^{j}\right)$ for some constants $C_{j}>0$, and,

$$
\left|\ell_{\eta}(x+y)-\ell_{\eta}(x)\right| \leq|y| \cdot Q_{1}(x), \quad\left|s_{\eta}(x+y)-s_{\eta}(x)\right| \leq|y| \cdot Q_{2}(x),
$$


for every $\eta \in \mathcal{H}_{0}, x$ and $y$ with $|y| \leq L \epsilon$ (see Lemma 3.2.3 of [8] for details). Thus $\sqrt{n}\left|Z_{n i}(\theta, \eta)\right| \lesssim Q_{1}\left(X_{i}\right)$ for every $i \leq n$ and $(\theta, \eta) \in \mathcal{F}$. Since $Q_{1}$ is $P_{\eta_{0}}$-square-integrable,

$$
\sum_{i=1}^{n} P_{0}\left(S_{n i} 1_{\left\{S_{n i}>\gamma\right\}}\right) \leq \sqrt{n} P_{\eta_{0}}\left(Q_{1} 1_{\left\{Q_{1}>\sqrt{n} \gamma\right\}}\right) \leq \gamma^{-1} P_{\eta_{0}} Q_{1}^{2} 1_{\left\{Q_{1}>\sqrt{n} \gamma\right\}}=o(1),
$$

for every $\gamma>0$, so the first condition of A.22 is satisfied.

Note that,

$$
\begin{aligned}
\mid Z_{n i}\left(\theta_{1}, \eta_{1}\right)- & Z_{n i}\left(\theta_{2}, \eta_{2}\right) \mid \\
& \leq\left|Z_{n i}\left(\theta_{1}, \eta_{1}\right)-Z_{n i}\left(\theta_{2}, \eta_{1}\right)\right|+\left|Z_{n i}\left(\theta_{2}, \eta_{1}\right)-Z_{n i}\left(\theta_{2}, \eta_{2}\right)\right| .
\end{aligned}
$$

The first term of the right-hand side of (A.25) is bounded by,

$$
\sup _{\eta \in \mathcal{H}_{0}}\left|Z_{n i}\left(\theta_{1}, \eta\right)-Z_{n i}\left(\theta_{2}, \eta\right)\right| \lesssim \sup _{\eta \in \mathcal{H}_{0}} \frac{1}{\sqrt{n}}\left|\dot{\ell}_{\theta_{1}, \eta, i}-\dot{\ell}_{\theta_{2}, \eta, i}\right| \lesssim \frac{\left|\theta_{1}-\theta_{2}\right|}{\sqrt{n}} Q_{2}\left(X_{i}\right) .
$$

For every $y$ with $|y| \leq L \epsilon$, let $\mathcal{S}_{y}=\left\{x \mapsto s_{\eta}(x-y): \eta \in \mathcal{H}_{0}\right\}$. Since the first and second derivatives of $x \mapsto s_{\eta}(x-y)$ are of order $O\left(x^{2}\right)$ and $O\left(x^{3}\right)$, (uniformly in $|y| \leq L \epsilon$ and $\left.\eta \in \mathcal{H}_{0}\right)$ and $\eta_{0}(x)=O\left(e^{-C x^{2}}\right)$ for some $C>0$ as $|x| \rightarrow \infty$, we have $\sup _{|y| \leq L \epsilon} \log N_{[]}\left(\delta, \mathcal{S}_{y}, L_{2}\left(P_{\eta_{0}}\right)\right) \lesssim \delta^{-1 / 2}$ for every small enough $\delta>0$ by Corollary 2.7.4 of [33] with $\alpha=r=2, d=1, V=1 / 2$ and a partition $\mathbb{R}=\cup_{j=-\infty}^{\infty}[j-1, j)$. Assume that some sufficiently small $\delta>0$ is given and we choose a sequence $\left(y_{j}\right)_{j=0}^{N_{\delta}}$ such that $-\epsilon L=y_{0}<y_{1}<\cdots<y_{N_{\delta}}=\epsilon L$ and $y_{j+1}-y_{j}<\delta$. Since $N_{\delta} \lesssim \delta^{-1}$ and $\log N_{[]}\left(\delta^{3 / 2}, \mathcal{S}_{y}, L_{2}\left(P_{\eta_{0}}\right)\right) \lesssim \delta^{-3 / 4}$, we can construct a partition $\left\{\mathcal{H}_{l}: 1 \leq l \leq \bar{N}_{\delta}\right\}$ of $\mathcal{H}_{0}$ by taking all intersections of sets in $N_{\delta}+1$ partitions, so that $\log \bar{N}_{\delta} \leq N_{\delta}$. $\log N_{[]}\left(\delta^{3 / 2}, \mathcal{S}_{y}, L_{2}\left(P_{\eta_{0}}\right)\right) \lesssim \delta^{-7 / 4}$ and

$$
\int \sup _{\eta_{1}, \eta_{2} \in \mathcal{H}_{l}}\left|s_{\eta_{1}}\left(x-y_{j}\right)-s_{\eta_{2}}\left(x-y_{j}\right)\right|^{2} d P_{\eta_{0}}(x) \leq \delta^{3},
$$

for every $l$ and $j$. Applying Lemma 2.2.2 of [33] with $\psi(x)=x^{2}$, we have,

$$
\int \max _{1 \leq j \leq N_{\delta}} \sup _{\eta_{1}, \eta_{2} \in \mathcal{H}_{l}}\left|s_{\eta_{1}}\left(x-y_{j}\right)-s_{\eta_{2}}\left(x-y_{j}\right)\right|^{2} d P_{\eta_{0}}(x) \lesssim \delta^{2},
$$

for every $l$.

Now, consider the second term of the right-hand side of A.25. For every $\theta \in B_{\epsilon}$ and $i \geq 1$, we can choose $j$ such that $\left|\theta^{T} Z_{i}-y_{j}\right| \leq \delta$. Then,

$$
\begin{aligned}
\left(Z_{n i}\left(\theta, \eta_{1}\right)-Z_{n i}\left(\theta, \eta_{2}\right)\right)^{2} & \lesssim \frac{1}{n}\left|\dot{\ell}_{\theta, \eta_{1}, i}-\dot{\ell}_{\theta, \eta_{2}, i}\right|^{2} \\
& \lesssim \frac{1}{n}\left|s_{\eta_{1}}\left(X_{i}-\theta^{T} Z_{i}\right)-s_{\eta_{2}}\left(X_{i}-\theta^{T} Z_{i}\right)\right|^{2} \\
& \lesssim \frac{\delta^{2}}{n} Q_{2}^{2}\left(X_{i}\right)+\frac{1}{n}\left|s_{\eta_{1}}\left(X_{i}-y_{j}\right)-s_{\eta_{2}}\left(X_{i}-y_{j}\right)\right|^{2}
\end{aligned}
$$

so we have,

$$
P_{0}\left(\sum_{i=1}^{n} \sup _{\theta \in B_{\epsilon}} \sup _{\eta_{1}, \eta_{2} \in \mathcal{H}_{l}}\left(Z_{n i}\left(\theta, \eta_{1}\right)-Z_{n i}\left(\theta, \eta_{2}\right)\right)^{2}\right) \lesssim \delta^{2},
$$


for every $l$.

Finally, the two bounds A.26 and A.28 combined with A.25, imply that,

$$
\sum_{i=1}^{n} P_{0} \sup _{\substack{\left|\theta_{1}-\theta_{2}\right| \leq \delta \\ \eta_{1}, \eta_{2} \in \mathcal{H}_{l}}}\left|Z_{n i}\left(\theta_{1}, \eta_{1}\right)-Z_{n i}\left(\theta_{2}, \eta_{2}\right)\right|^{2} \lesssim \delta^{2},
$$

for every $l$. Since $N\left(\delta, B_{\epsilon},|\cdot|\right) \lesssim \delta^{-p}$, a partition satisfying A.21 can be constructed by product sets of each partition of $B_{\epsilon}$ and $\mathcal{H}_{0}$, the order of which is bounded as (for some constant $K>0$ ),

$$
\log N_{[]}^{n}(\delta, \mathcal{F}) \lesssim \log \bar{N}_{K \delta}+\log \delta^{-p} \lesssim \delta^{-7 / 4}
$$

so the second condition of A.22 is satisfied.

\section{A.1.8 Proof of asymptotic tightness in Corollary 3.2}

We follow the steps of the proof of asymptotic tightness in Corollary 3.1. Without loss of generality we assume that $\theta_{0}=0$, and define $Z_{n i}(\theta, \eta), S_{n i}, \mathcal{F}$ and $N_{\square}^{n}(\delta, \mathcal{F})$ as in the proof of Corollary 3.1. The first condition of A.22 is proved by replacing $Q_{j}$ 's as constant functions. Inequalities A.25 and A.26 are shown to hold in the same way.

Let $\mathcal{S}=\left\{x \mapsto s_{\eta}(x): \eta \in \mathcal{H}_{0}\right\}$. Applying Theorem 2.7.1 of [33] with $\alpha=d=1$, we have $\log N\left(\delta, \mathcal{S},\|\cdot\|_{\infty}\right) \lesssim \delta^{-1}$. This implies that there exists a partition $\left\{\mathcal{H}_{l}: 1 \leq l \leq \bar{N}_{\delta}\right\}$ of $\mathcal{H}_{0}$ such that $\bar{N}_{\delta} \lesssim \delta^{-1}$ and,

$$
\sup _{\eta_{1}, \eta_{2} \in \mathcal{H}_{l}} \sup _{x \in \mathbb{D}}\left|s_{\eta_{1}}(x)-s_{\eta_{2}}(x)\right|<\delta,
$$

for every $l$. Thus, A.28 holds. Replacing the entropy bound A.29 by,

$$
\log N_{[]}^{n}(\delta, \mathcal{F}) \lesssim \log \bar{N}_{K \delta}+\log \delta^{-p} \lesssim \delta^{-1},
$$

we follow the remainder of the proof of Corollary 3.1 .

\section{A.2 Proofs for Section 4}

\section{A.2.1 Proof of 4.7}

Since,

$$
\begin{aligned}
& \sup _{\eta \in \mathcal{H}^{N}}\left|\frac{1}{n} P_{0}^{(n)}\left(\ell_{\theta, \eta}^{(n)}-\ell_{\theta_{0}, \eta}^{(n)}\right)+\frac{1}{2}\left(\theta-\theta_{0}\right)^{T} V_{n, \eta}\left(\theta-\theta_{0}\right)\right| \\
& \leq \sup _{i, \eta}\left|P_{0}\left(\log \frac{\psi_{\eta}\left(X_{i}-Z_{i}^{T} \theta \mid W_{i}\right)}{\psi_{\eta}\left(X_{i}-Z_{i}^{T} \theta_{0} \mid W_{i}\right)}\right)+\frac{1}{2}\left(\theta-\theta_{0}\right)^{T} Z_{i} v_{\eta}\left(W_{i}\right) Z_{i}^{T}\left(\theta-\theta_{0}\right)\right|,
\end{aligned}
$$

where $i$ runs over the integers and $\eta$ over $\mathcal{H}^{N}$, it suffices to show that,

$$
\sup _{w} \sup _{\eta \in \mathcal{H}^{N}}\left|\int \log \frac{\psi_{\eta}(x-y \mid w)}{\psi_{\eta}(x \mid w)} d \Psi_{\eta_{0}}^{w}(x)+\frac{1}{2} y^{T} v_{\eta}(w) y\right|=o\left(|y|^{2}\right),
$$


as $|y| \rightarrow 0$.

Let $A=\left\{x=\left(x_{1}, \ldots, x_{m}\right): x_{1}>0\right\}$ and $A^{-}=\{x:-x \in A\}$. Note that,

$$
\psi_{\eta}(x \mid w)=\psi_{\eta}(-x \mid w)=\psi_{\eta}(x \mid-w)=\psi_{\eta}(-x \mid-w),
$$

by the symmetry of $f$ and $G$. Thus, for $\eta \in \mathcal{H}^{N}$,

$$
\begin{aligned}
& \int \log \frac{\psi_{\eta}(x-y \mid w)}{\psi_{\eta}(x \mid w)} d \Psi_{\eta_{0}}^{w}(x)=\int \log \frac{\psi_{\eta}(x-y \mid w)}{\psi_{\eta}(x \mid w)} \psi_{\eta_{0}}(x \mid w) d \mu(x) \\
& =\int_{A^{-}} \log \frac{\psi_{\eta}(x-y / 2 \mid w)}{\psi_{\eta}(x+y / 2 \mid w)} \psi_{\eta_{0}}(x+y / 2 \mid w) d \mu(x) \\
& \quad+\int_{A} \log \frac{\psi_{\eta}(x-y / 2 \mid w)}{\psi_{\eta}(x+y / 2 \mid w)} \psi_{\eta_{0}}(x+y / 2 \mid w) d \mu(x) \\
& =\int_{A} \log \frac{\psi_{\eta}(-x-y / 2 \mid w)}{\psi_{\eta}(-x+y / 2 \mid w)} \psi_{\eta_{0}}(-x+y / 2 \mid w) d \mu(x) \\
& \quad+\int_{A} \log \frac{\psi_{\eta}(x-y / 2 \mid w)}{\psi_{\eta}(x+y / 2 \mid w)} \psi_{\eta_{0}}(x+y / 2 \mid w) d \mu(x) \\
& =-\int_{A}\left[\ell_{\eta}\left(x-\frac{y}{2} \mid w\right)-\ell_{\eta}\left(x+\frac{y}{2} \mid w\right)\right] \\
& \quad \times\left[\psi_{\eta_{0}}\left(x-\frac{y}{2} \mid w\right)-\psi_{\eta_{0}}\left(x+\frac{y}{2} \mid w\right)\right] d \mu(x) .
\end{aligned}
$$

The last integral of A.31 is equal to,

$$
\begin{aligned}
-\int_{0}^{1} \int_{0}^{1} \int_{A} y^{T} s_{\eta}(x & +r(y, t) \mid w) s_{\eta_{0}}^{T}(x+r(y, s) \mid w) y \\
& \times \psi_{\eta_{0}}(x+r(y, s) \mid w) d \mu(x) d t d s
\end{aligned}
$$

by Taylor expansion, where $r(y, t)=(t-1 / 2) y$. Since,

$$
v_{\eta}(w)=2 \int_{A} s_{\eta}(x \mid w) s_{\eta_{0}}^{T}(x \mid w) d \Psi_{\eta_{0}}^{w}(x),
$$

the left-hand side of A.30, for fixed $w$ and $\eta$, is equal to,

$$
\begin{aligned}
-y^{T}\left\{\int_{0}^{1} \int_{0}^{1} \int_{A}\right. & {\left[s_{\eta}(x+r(y, t) \mid w) s_{\eta_{0}}^{T}(x+r(y, s) \mid w) \psi_{\eta_{0}}(x+r(y, s) \mid w)\right.} \\
& \left.\left.-s_{\eta}(x \mid w) s_{\eta_{0}}^{T}(x \mid w) \psi_{\eta_{0}}(x \mid w)\right] d \mu(x) d t d s\right\} y .
\end{aligned}
$$

The integrand of the last display is equal to $A_{\eta}(x, y, w)+B_{\eta}(x, y, w)$, where,

$$
\begin{aligned}
A_{\eta}(x, y, w) & =s_{\eta}(x+r(y, t) \mid w) \\
& \times\left\{s_{\eta_{0}}^{T}(x+r(y, s) \mid w) \psi_{\eta_{0}}(x+r(y, s) \mid w)-s_{\eta_{0}}^{T}(x \mid w) \psi_{\eta_{0}}(x \mid w)\right\},
\end{aligned}
$$

and,

$$
B_{\eta}(x, y, w)=\left\{s_{\eta}(x+r(y, t) \mid w)-s_{\eta}(x \mid w)\right\} s_{\eta_{0}}^{T}(x \mid w) \psi_{\eta_{0}}(x \mid w)
$$

(dependence on $t$ and $s$ is abbreviated for simplicity). Let $g_{\eta, j}(x \mid w)=\partial \ell_{\eta}(x \mid w) / \partial x_{j}$ and $e_{j}$ be the $j$ th unit vector in $\mathbb{R}^{m}$. By (4.4), it is easy to prove that,

$$
\sup _{w} \sup _{\eta \in \mathcal{H}^{N}} \sup _{t, s \in[0,1]} \int_{A}\left|e_{i}^{T} B_{\eta}(x, y, w) e_{j}\right| d \mu(x)=o(1),
$$


as $|y| \rightarrow 0$. Also, by (4.4),

$$
\left|e_{i}^{T}\left[\frac{\partial\left(s_{\eta_{0}} \psi_{\eta_{0}}\right)}{\partial x}(x \mid w)\right] e_{j}\right| \leq\left(Q+Q^{2}\right)(x, w) \psi_{\eta_{0}}(x \mid w),
$$

for every $i, j \leq m$. Thus, $\left|e_{i}^{T} A_{\eta}(x, y, w) e_{j}\right|$ is bounded by,

$$
\begin{aligned}
& |y| \cdot\left|g_{\eta, i}(x+r(y, t) \mid w)\right| \cdot \int_{0}^{1}\left(Q+Q^{2}\right)(x+r(y, s) u, w) \psi_{\eta_{0}}(x+r(y, s) u \mid w) d u \\
& \leq|y|(1+|y|) \cdot \int_{0}^{1}\left\{\left(Q^{2}+Q^{3}\right)(x+r(y, s) u, w) \psi_{\eta_{0}}(x+r(y, s) u \mid w)\right\} d u
\end{aligned}
$$

where the inequality in the second line holds because,

$$
\begin{aligned}
& \left|g_{\eta, i}(x+r(y, t) \mid w)\right| \\
& \leq\left|g_{\eta, i}(x+r(y, t) \mid w)-g_{\eta, i}(x+r(y, s) u \mid w)\right|+\left|g_{\eta, i}(x+r(y, s) u \mid w)\right| \\
& \leq(1+|y|) Q(x+r(y, s) u, w) .
\end{aligned}
$$

Therefore,

$$
\begin{aligned}
& \sup _{s, t \in[0,1]} \sup _{\eta \in \mathcal{H}^{N}} \int_{A}\left|e_{i}^{T} A_{\eta}(x, y, w) e_{j}\right| d \mu(x) d t d s \\
& \leq|y|(1+|y|) \int\left(Q^{2}+Q^{3}\right)(x, w) d \Psi_{\eta_{0}}^{w}(x),
\end{aligned}
$$

which is $o(1)$, uniformly in $w$, as $|y| \rightarrow 0$.

\section{A.2.2 Proof of $(4.8)$}

To prove (4.8), it suffices to show,

$$
\lim _{n \rightarrow \infty} \sup _{\eta \in \mathcal{H}_{n}} d_{W_{i}}\left(\eta, \eta_{0}\right)=0
$$

for every $i \geq 1$ because $W_{i}$ is contained in a compact set, 4.3) holds, and (4.5) is uniformly equicontinuous (note that equicontinuity on a compact domain is equivalent to uniform equicontinuity). For given $i \geq 1$, since $\sup _{\eta \in \mathcal{H}^{N}} d_{W_{i}}\left(\eta, \eta_{0}\right)<\infty$ by 4.4), we can choose $\eta_{n} \in \mathcal{H}_{n}$, for large enough $n$ such that,

$$
\sup _{\eta \in \mathcal{H}_{n}} d_{W_{i}}\left(\eta, \eta_{0}\right)<d_{W_{i}}\left(\eta_{n}, \eta_{0}\right)+n^{-1} .
$$

Note that $h_{n}\left(\eta_{n}, \eta_{0}\right) \rightarrow 0$ by the definition of $\mathcal{H}_{n}$. Since,

$$
h_{n}^{2}\left(\eta_{n}, \eta_{0}\right)=\frac{1}{n} \sum_{j=1}^{n} h^{2}\left(\psi_{\eta_{n}}\left(\cdot \mid W_{j}\right), \psi_{\eta_{0}}\left(\cdot \mid W_{j}\right)\right),
$$

$W_{j}$ is contained in a compact set, 4.3 holds, and 4.5 is uniformly equicontinuous, we have $\lim _{n \rightarrow \infty} h\left(\psi_{\eta_{n}}\left(\cdot \mid W_{j}\right), \psi_{\eta_{0}}\left(\cdot \mid W_{j}\right)\right)=0$ for every $j \geq 1$. Thus, it suffices to show that $d_{W_{i}}\left(\eta_{n}, \eta_{0}\right) \rightarrow 0$. For simplicity, we write $W_{i}=w$ in the remainder of this proof. 
We first prove that $\lim _{n \rightarrow \infty} \ell_{\eta_{n}}(x \mid w)=\ell_{\eta_{0}}(x \mid w)$ for every $x$. Suppose $\ell_{\eta_{n}}(x \mid w) \nrightarrow$ $\ell_{\eta_{0}}(x \mid w)$ for some $x$. Then we can choose an $\epsilon>0$ and a subsequence $m(n)$ such that $m(n) \geq N$ and $\left|\ell_{\eta_{m(n)}}(x \mid w)-\ell_{\eta_{0}}(x \mid w)\right|>\epsilon$ for every $n$. Note that $x \mapsto \ell_{\eta}(x \mid w)$ is continuously differentiable and its derivative is bounded componentwise by a continuous function $x \mapsto Q(x, w)$ uniformly in $\eta \in \mathcal{H}^{N}$ by (4.4). Thus we can choose a $\delta>0$ such that $\left|\ell_{\eta_{m(n)}}(y \mid w)-\ell_{\eta_{0}}(y \mid w)\right|>\epsilon / 2$ for every $n \geq 1$ and a $y$ with $|y-x|<\delta$. Note that $\delta>0$ can be chosen sufficiently small so that $\psi_{\eta_{0}}(y \mid w)>\psi_{\eta_{0}}(x \mid w) / 2$ for every $y$ with $|y-x|<\delta$. Since,

$$
\ell_{\eta_{m(n)}}(y \mid w)-\ell_{\eta_{0}}(y \mid w)=2 \log \sqrt{\psi_{\eta_{m(n)}}(y \mid w) / \psi_{\eta_{0}}(y \mid w)},
$$

there exists a $\bar{\epsilon}>0$ such that,

$$
\left|1-\sqrt{\psi_{\eta_{m(n)}}(y \mid w) / \psi_{\eta_{0}}(y \mid w)}\right|>\bar{\epsilon},
$$

for every $n \geq 1$ and $y$ with $|y-x|<\delta$. Since,

$$
\begin{aligned}
h^{2}\left(\psi_{\eta_{m(n)}}(\cdot \mid w), \psi_{\eta_{0}}(\cdot \mid w)\right) & \geq \int_{\{y:|y-x|<\delta\}}\left(1-\sqrt{\frac{\psi_{\eta_{m(n)}}}{\psi_{\eta_{0}}}(y \mid w)}\right)^{2} d \Psi_{\eta_{0}}^{w}(y) \\
& \geq \bar{\epsilon}^{2} \int_{\{y:|y-x|<\delta\}} \frac{\psi_{\eta_{0}}(x \mid w)}{2} d y \geq \gamma
\end{aligned}
$$

for some $\gamma>0$ and every $n \geq 1$, the above contradicts the fact that $h\left(\psi_{\eta_{n}}, \psi_{\eta_{0}}\right) \rightarrow 0$, so we conlude that $\ell_{\eta_{n}}(x \mid w) \rightarrow \ell_{\eta_{0}}(x \mid w)$ for all $x$.

Let $e_{j}$ be the $j$ th unit vector in $\mathbb{R}^{m}$ and $g_{\eta, j}(x \mid w)=\partial \ell_{\eta}(x \mid w) / \partial x_{j}$. Then as $y \rightarrow 0$ in $\mathbb{R}$,

$$
\begin{aligned}
\sup _{\eta \in \mathcal{H}^{N}} \mid \int & \left.\frac{\ell_{\eta}\left(x+y e_{j} \mid w\right)-\ell_{\eta}(x \mid w)}{y}-g_{\eta_{0}, j}(x \mid w)\right)^{2} \\
& -\left(g_{\eta, j}(x \mid w)-g_{\eta_{0}, j}(x \mid w)\right)^{2} d \Psi_{\eta_{0}}^{w}(x) \mid \\
= & \sup _{\eta \in \mathcal{H}^{N}} \mid \int\left\{\int_{0}^{1}\left|g_{\eta, j}\left(x+t y e_{j} \mid w\right)-g_{\eta, j}(x \mid w)\right| d t\right. \\
& \left.\times\left[\frac{\ell_{\eta}\left(x+y e_{j} \mid w\right)-\ell_{\eta}(x \mid w)}{y}-g_{\eta, j}(x \mid w)+2 g_{\eta_{0}, j}(x \mid w)\right]\right\} d \Psi_{\eta_{0}}^{w}(x) \mid \\
\leq & |y| \int Q(x, w) \mid \frac{\ell_{\eta}\left(x+y e_{j} \mid w\right)-\ell_{\eta}(x \mid w)}{y}-g_{\eta, j}(x \mid w) \\
& +2 g_{\eta_{0}, j}(x \mid w) \mid d \Psi_{\eta_{0}}^{w}(x) \\
= & o(1),
\end{aligned}
$$

where the last line holds by (4.4). The Moore-Osgood theorem enables the interchange of 
the two limits in the following equality:

$$
\begin{aligned}
& \lim _{n \rightarrow \infty} \int\left\{g_{\eta_{n}, j}(x \mid w)-g_{\eta_{0}, j}(x \mid w)\right\}^{2} d \Psi_{\eta_{0}}^{w}(x) \\
& =\lim _{n \rightarrow \infty} \lim _{y \rightarrow 0} \int\left\{\frac{\ell_{\eta_{n}}\left(x+y e_{j} \mid w\right)-\ell_{\eta_{0}}(x \mid w)}{y}-g_{\eta_{0}, j}(x \mid w)\right\}^{2} d \Psi_{\eta_{0}}^{w}(x) \\
& =\lim _{y \rightarrow 0} \lim _{n \rightarrow \infty} \int\left\{\frac{\ell_{\eta_{n}}\left(x+y e_{j} \mid w\right)-\ell_{\eta_{0}}(x \mid w)}{y}-g_{\eta_{0}, j}(x \mid w)\right\}^{2} d \Psi_{\eta_{0}}^{w}(x) \\
& =\int\left\{g_{\eta_{0}, j}(x \mid w)-g_{\eta_{0}, j}(x \mid w)\right\}^{2} d \Psi_{\eta_{0}}^{w}(x)=0 .
\end{aligned}
$$

Conclude that $d_{w}\left(\eta_{n}, \eta_{0}\right)=o(1)$.

\section{A.2.3 Proof of Lemma 4.1}

Let $\psi_{\eta, j}\left(x_{j} \mid w_{j}\right)$ be the marginal density of the $j$ th coordinate, that is $\psi_{\eta, j}\left(x_{j} \mid w_{j}\right)=$ $\int f\left(x_{j}-b^{T} w_{j}\right) d G(b)$. Since $G_{0}$ is thick at 0 and $f_{0}$ is continuous and positive at 0 , there exists a $\gamma>0$ such that $\inf _{\left|x_{j}\right| \leq \gamma} \inf _{w_{j}} \psi_{\eta_{0}, j}\left(x_{j} \mid w_{j}\right)>0$. Thus, as in A.7), there exist constants $\widetilde{C}>0$ and $\delta>0$ such that,

$$
h^{2}\left(p_{\theta, \eta, i}, p_{\theta_{0}, \eta_{0}, i}\right) \geq \widetilde{C}^{2}\left(\delta \wedge\left|\left(\theta-\theta_{0}\right)^{T} Z_{i j}\right|\right)^{2}
$$

for every $j \leq m$. Since $\max _{j \leq m}\left|\left(\theta-\theta_{0}\right)^{T} Z_{i j}\right| \geq\left|\left(\theta-\theta_{0}\right)^{T} Z_{i}\right| / \sqrt{m}$,

$$
h^{2}\left(p_{\theta, \eta, i}, p_{\theta_{0}, \eta_{0}, i}\right) \geq C^{2}\left(\delta \wedge\left|\left(\theta-\theta_{0}\right)^{T} Z_{i}\right|\right)^{2}
$$

where $C=\widetilde{C} / \sqrt{m}$. Let $\epsilon>0$ be a constant such that $\epsilon<a C \delta$, where $a^{2}=\liminf \rho_{n} \rho_{\min }\left(\mathbf{Z}_{n}\right) /\left(2 m L^{2}\right)$,

For a given large enough $n$, fix $\eta \in \mathcal{H}_{n}$ with $h_{n}\left((\theta, \eta),\left(\theta_{0}, \eta_{0}\right)\right)<\epsilon$. Let $\mathbb{N}_{\delta, n}=\{i \leq$ $\left.n:\left|\left(\theta-\theta_{0}\right)^{T} Z_{i}\right| \geq \delta\right\}$ and let $N_{\delta, n}$ denote its cardinality. Then, the last display implies,

$$
\begin{aligned}
\epsilon^{2} \geq h_{n}^{2}\left((\theta, \eta),\left(\theta_{0}, \eta_{0}\right)\right) & \geq \frac{C^{2}}{n} \sum_{i=1}^{n}\left(\left|\left(\theta-\theta_{0}\right)^{T} Z_{i}\right| \wedge \delta\right)^{2} \\
& \geq \frac{C^{2} N_{\delta, n} \delta^{2}}{n}+\frac{C^{2}}{n} \sum_{i \notin \mathbb{N}_{\delta, n}}\left|\left(\theta-\theta_{0}\right)^{T} Z_{i}\right|^{2} .
\end{aligned}
$$

The first term of $\mathrm{A} .32$ is greater than $N_{\delta, n} \epsilon^{2} /\left(n a^{2}\right)$ since $\epsilon<a C \delta$, which implies $N_{\delta, n} / n<$ $a^{2}$. On the other hand, for the second term of A.32, note that,

$$
\sum_{i \notin \mathbb{N}_{\delta, n}}\left|\left(\theta-\theta_{0}\right)^{T} Z_{i}\right|^{2} \geq \sum_{i=1}^{n}\left|\left(\theta-\theta_{0}\right)^{T} Z_{i}\right|^{2}-N_{\delta, n} \max _{i}\left|\left(\theta-\theta_{0}\right)^{T} Z_{i}\right|^{2} .
$$

Since $\sum_{i=1}^{n}\left|\left(\theta-\theta_{0}\right)^{T} Z_{i}\right|^{2} \geq n\left|\theta-\theta_{0}\right|^{2} \rho_{\min }\left(\mathbf{Z}_{n}\right)$ and $\max _{i}\left|\left(\theta-\theta_{0}\right)^{T} Z_{i}\right|^{2} \leq m L^{2}\left|\theta-\theta_{0}\right|^{2}$, we have,

$$
\frac{C^{2}}{n} \sum_{i \notin \mathbb{N}_{\delta, n}}\left|\left(\theta-\theta_{0}\right)^{T} Z_{i}\right|^{2} \geq C^{2}\left|\theta-\theta_{0}\right|^{2}\left(\rho_{\min }\left(\mathbf{Z}_{n}\right)-m L^{2} \frac{N_{\delta, n}}{n}\right) .
$$


Since $N_{\delta, n} / n<a^{2}$ and $a^{2}=\liminf _{n} \rho_{\min }\left(\mathbf{Z}_{n}\right) /\left(2 m L^{2}\right)$, A.32 and A.33 together imply $\left|\theta-\theta_{0}\right|^{2} \leq K_{1} \epsilon^{2}$, where $K_{1}=2 /\left(C^{2} \rho_{\min }\left(\mathbf{Z}_{n}\right)\right)$.

The proof would be complete if we show that $h_{n}\left(\eta, \eta_{0}\right)<K \epsilon$ for some constant $K>0$. Note that for every $i$,

$$
\begin{aligned}
h_{n}^{2}\left(\eta, \eta_{0}\right) & =\frac{1}{n} \sum_{i=1}^{n} h^{2}\left(p_{\theta, \eta, i}, p_{\theta, \eta_{0}, i}\right) \\
& \leq \frac{2}{n} \sum_{i=1}^{n}\left(h^{2}\left(p_{\theta, \eta, i}, p_{\theta_{0}, \eta_{0}, i}\right)+h^{2}\left(p_{\theta_{0}, \eta_{0}, i}, p_{\theta, \eta_{0}, i}\right)\right) \\
& \left.=\frac{2}{n} \sum_{i=1}^{n} h^{2}\left(p_{\theta, \eta_{0}, i}, p_{\theta_{0}, \eta_{0}, i}\right)+2 h_{n}^{2}\left((\theta, \eta), \theta_{0}, \eta_{0}\right)\right) \\
& \leq \frac{2}{n} \sum_{i=1}^{n} h^{2}\left(p_{\theta, \eta_{0}, i}, p_{\theta_{0}, \eta_{0}, i}\right)+2 \epsilon^{2},
\end{aligned}
$$

Note also that,

$$
\frac{\partial}{\partial \theta} \sqrt{p_{\theta, \eta, i}(x)}=\frac{\frac{\partial}{\partial \theta} p_{\theta, \eta, i}(x)}{2 \sqrt{p_{\theta, \eta, i}(x)}}=\frac{1}{2} Z_{i} s_{\eta}\left(x-Z_{i}^{T} \theta \mid W_{i}\right) \sqrt{p_{\theta, \eta, i}(x)}
$$

Thus, with $\theta(t)=\theta_{0}+t\left(\theta-\theta_{0}\right)$,

$$
\begin{aligned}
& h^{2}\left(p_{\theta, \eta_{0}, i}, p_{\theta_{0}, \eta_{0}, i}\right)=\int\left(\sqrt{p_{\theta, \eta_{0}, i}(x)}-\sqrt{p_{\theta_{0}, \eta_{0}, i}(x)}\right)^{2} d \mu(x) \\
& \leq \frac{1}{4} \iint_{0}^{1}\left|\left(\theta-\theta_{0}\right)^{T} Z_{i} s_{\eta_{0}}\left(x-Z_{i}^{T} \theta(t) \mid W_{i}\right)\right|^{2} p_{\theta(t), \eta_{0}, i}(x) d t d \mu(x) \\
& =\frac{1}{4} \int\left|\left(\theta-\theta_{0}\right)^{T} Z_{i} s_{\eta_{0}}\left(x \mid W_{i}\right)\right|^{2} d \Psi_{\eta_{0}}^{W_{i}}(x) \\
& \leq K_{2}\left|\theta-\theta_{0}\right|^{2},
\end{aligned}
$$

for some $K_{2}>0$ by (4.4), where the inequality in the second line of A.34 holds by Jensen's inequality. Thus, $h_{n}^{2}\left(\eta, \eta_{0}\right) \leq 2 K_{2}\left|\theta-\theta_{0}\right|^{2}+2 \epsilon^{2} \leq 2\left(K_{1} K_{2}+1\right) \epsilon^{2}$.

\section{A.2.4 Proof of Lemma 4.2}

Let $\eta_{1}=\left(f_{1}, G_{1}\right)$ and $\eta_{2}=\left(f_{2}, G_{2}\right)$ be elements of $\mathcal{H}_{0}, \theta_{1}, \theta_{2} \in \Theta$ and let $\eta_{12}=\left(f_{1}, G_{2}\right)$. Since (4.10) and (4.11) hold, it can be shown, in a manner similar to (A.34), that,

$$
\sup _{i \geq 1} \sup _{\eta \in \mathcal{H}_{0}} h\left(p_{\theta_{1}, \eta, i}, p_{\theta_{2}, \eta, i}\right) \leq C\left|\theta_{1}-\theta_{2}\right|,
$$

for some constant $C>0$. Recall that $h(P * G, Q * G) \leq h(P, Q)$ for arbitrary probability measures $P, Q$ and $G$ (where $*$ denotes convolution). Then,

$$
\begin{aligned}
h\left(p_{\theta_{1}, \eta_{1}, i},\right. & \left.p_{\theta_{2}, \eta_{2}, i}\right) \leq h\left(p_{\theta_{1}, \eta_{1}, i}, p_{\theta_{1}, \eta_{2}, i}\right)+h\left(p_{\theta_{1}, \eta_{2}, i}, p_{\theta_{2}, \eta_{2}, i}\right) \\
& \leq h\left(\psi_{\eta_{1}}\left(\cdot \mid W_{i}\right), \psi_{\eta_{2}}\left(\cdot \mid W_{i}\right)\right)+C\left|\theta_{1}-\theta_{2}\right| \\
& \leq h\left(\psi_{\eta_{1}}\left(\cdot \mid W_{i}\right), \psi_{\eta_{12}}\left(\cdot \mid W_{i}\right)\right)+h\left(\psi_{\eta_{12}}\left(\cdot \mid W_{i}\right), \psi_{\eta_{2}}\left(\cdot \mid W_{i}\right)\right)+C\left|\theta_{1}-\theta_{2}\right| \\
& \leq h\left(\psi_{\eta_{1}}\left(\cdot \mid W_{i}\right), \psi_{\eta_{12}}\left(\cdot \mid W_{i}\right)\right)+h\left(f_{1}^{m}, f_{2}^{m}\right)+C\left|\theta_{1}-\theta_{2}\right|, \\
& =h\left(\psi_{\eta_{1}}\left(\cdot \mid W_{i}\right), \psi_{\eta_{12}}\left(\cdot \mid W_{i}\right)\right)+o(1),
\end{aligned}
$$


as $h\left(f_{1}, f_{2}\right) \vee\left|\theta_{1}-\theta_{2}\right| \rightarrow 0$, where $f^{m}$ is the $m$-fold product density of $f$. To prove 4.13, it now suffices to show that $h\left(\psi_{\eta_{1}}\left(\cdot \mid W_{i}\right), \psi_{\eta_{12}}\left(\cdot \mid W_{i}\right)\right)=o(1)$ as $d_{W}\left(G_{1}, G_{2}\right) \rightarrow 0$.

By 4.12, there exists a constant $C_{1}>0$ such that,

$$
\sup _{f \in \mathcal{F}_{0}} \sup _{x, w}\left|\prod_{j=1}^{m} f\left(x_{j}-b_{1}^{T} w_{j}\right)-\prod_{j=1}^{m} f\left(x_{j}^{T}-b_{2} w_{j}\right)\right| \leq C_{1}\left|b_{1}-b_{2}\right|,
$$

for every $b_{1}, b_{2} \in\left[-M_{b}, M_{b}\right]^{q}$. So by Theorem 2 of [15] (the equivalence of Lévy-Prohorov and Wasserstein metrics),

$$
\sup _{x, w}\left|\psi_{\eta_{1}}(x \mid w)-\psi_{\eta_{12}}(x \mid w)\right| \leq C_{2} d_{W}\left(G_{1}, G_{2}\right),
$$

for some $C_{2}>0$ that depends only on $C_{1}$. Since $w$ ranges over a compact set, inequality A.35 and uniform tightness of $\mathcal{F}_{0}$ imply that,

$$
\sup _{w} d_{V}\left(\psi_{\eta_{1}}(\cdot \mid w), \psi_{\eta_{12}}(\cdot \mid w)\right) \rightarrow 0,
$$

as $d_{W}\left(G_{1}, G_{2}\right) \rightarrow 0$. Since $h^{2} \leq d_{V}$, this completes the proof of 4.13.

To prove (4.14), write,

$$
K\left(p_{\theta_{0}, \eta_{0}, i}, p_{\theta, \eta, i}\right)=K\left(\ell_{\theta_{0}, \eta_{0}, i}, \ell_{\theta_{0}, \eta, i}\right)+P_{0}\left(\ell_{\theta_{0}, \eta, i}-\ell_{\theta, \eta, i}\right) .
$$

Under conditions 4.10 and 4.11, $K\left(p_{\theta_{0}, \eta_{0}, i}, p_{\theta_{0}, \eta, i}\right)$ is bounded by,

$$
C_{3} h^{2}\left(p_{\theta_{0}, \eta_{0}, i}, p_{\theta_{0}, \eta, i}\right) \log \left(\frac{1}{h\left(p_{\theta_{0}, \eta_{0}, i}, p_{\theta_{0}, \eta, i}\right)}\right),
$$

for some constant $C_{3}>0$, by Theorem 5 of [37], which converges to 0 as $h\left(f, f_{0}\right) \vee$ $d_{W}\left(G, G_{0}\right) \rightarrow 0$ by 4.13 . Also, by (4.11),

$$
\sup _{\eta \in \mathcal{H}_{0}} P_{0}\left(\ell_{\theta_{0}, \eta, i}-\ell_{\theta, \eta, i}\right) \leq C_{4}\left|\theta-\theta_{0}\right|
$$

for some $C_{4}>0$, and so $K\left(p_{\theta_{0}, \eta_{0}, i}, p_{\theta, \eta, i}\right) \rightarrow 0$ as $\left|\theta-\theta_{0}\right| \vee h\left(f, f_{0}\right) \vee d_{W}\left(G, G_{0}\right) \rightarrow 0$. Similarly,

$$
V\left(p_{\theta_{0}, \eta_{0}, i}, p_{\theta, \eta, i}\right) \leq 2 P_{0}\left(\ell_{\theta_{0}, \eta_{0}, i}-\ell_{\theta_{0}, \eta, i}\right)^{2}+2 P_{0}\left(\ell_{\theta_{0}, \eta, i}-\ell_{\theta, \eta, i}\right)^{2}
$$

and $P_{0}\left(\ell_{\theta_{0}, \eta_{0}, i}-\ell_{\theta_{0}, \eta, i}\right)^{2}$ is bounded by,

$$
C_{5} h^{2}\left(p_{\theta_{0}, \eta_{0}, i}, p_{\theta_{0}, \eta, i}\right)\left\{\log \left(\frac{1}{h\left(p_{\theta_{0}, \eta_{0}, i}, p_{\theta_{0}, \eta, i}\right)}\right)\right\}^{2},
$$

for some $C_{5}>0$ by Theorem 5 of [37]. In addition,

$$
P_{0}\left(\ell_{\theta_{0}, \eta, i}-\ell_{\theta, \eta, i}\right)^{2} \leq C_{6}|\theta-\theta|^{2}
$$

for some $C_{6}>0$. Thus, $V\left(p_{\theta_{0}, \eta_{0}, i}, p_{\theta, \eta, i}\right) \rightarrow 0$ as $\left|\theta-\theta_{0}\right|, h\left(f, f_{0}\right)$ and $d_{W}\left(G, G_{0}\right)$ go to zero. 


\section{A.2.5 Proof of 4.15}

For $x \in \mathbb{R}^{m}$ and $w \in \mathbb{R}^{q \times m}$ note that,

$$
\begin{aligned}
\psi_{\eta}(x \mid w) & =\int \prod_{j=1}^{m} \int \phi_{\sigma}\left(x-z-b^{T} w_{j}\right) d F(z, \sigma) d G(b) \\
& =\int \prod_{j=1}^{m} \int \frac{1}{\sqrt{2 \pi} \sigma} \exp \left(-\frac{\left(x_{j}-z-b^{T} w_{j}\right)^{2}}{2 \sigma^{2}}\right) d F(z, \sigma) d G(b) \\
& \leq\left(2 \pi \sigma_{1}^{2}\right)^{-m / 2} \exp \left(-\frac{|x|^{2}}{2 \sigma_{2}^{2}}+K_{1}\right) \\
& \leq C_{3} \exp \left(-C_{4}|x|^{2}\right)
\end{aligned}
$$

for $C_{3}=\left(2 \pi \sigma_{1}^{2}\right)^{-m / 2}, C_{4}<1 /\left(2 \sigma_{2}^{2}\right)$ and large enough $|x|$, where $K_{1}$ is a constant. In the same way,

$$
\begin{aligned}
\psi_{\eta}(x \mid w) & \geq\left(2 \pi \sigma_{2}^{2}\right)^{-m / 2} \exp \left(-\frac{|x|^{2}}{2 \sigma_{1}^{2}}+K_{2}\right) \\
& \geq C_{1} \exp \left(-C_{2}|x|^{2}\right)
\end{aligned}
$$

for $C_{1}=\left(2 \pi \sigma_{2}^{2}\right)^{-m / 2}, C_{2}>1 /\left(2 \sigma_{1}^{2}\right)$ and large enough $|x|$, where $K_{2}$ is a constant.

\section{A.2.6 Proof of the equicontinuity of 4.5) in Corollary 4.1}

To prove the equicontinuity of (4.5), it is sufficient to show that the partial derivatives of $w \mapsto d_{w}^{2}\left(\eta_{1}, \eta_{2}\right)$ and $w \mapsto h^{2}\left(\psi_{\eta_{1}}(\cdot \mid w), \psi_{\eta_{2}}(\cdot \mid w)\right)$ are bounded by a constant uniformly in $\eta_{1}, \eta_{2} \in \mathcal{H}_{0}$. Since every $G$ is compactly supported, partial derivatives of $w \mapsto s_{\eta}(x \mid w)$ and $w \mapsto \psi_{\eta_{0}}(x \mid w)$ are bounded by a constant multiple of partial derivatives of $x \mapsto s_{\eta}(x \mid w)$ and $x \mapsto \psi_{\eta_{0}}(x \mid w)$, which are bounded by $Q(x, w)$ and $Q(x, w) \psi_{\eta_{0}}(x \mid w)$, respectively. Since $s_{\eta}(x, w)$ is also bounded by $Q(x, w)$ for every $\eta \in \mathcal{H}_{0}$, the partial derivative of,

$$
w \mapsto d_{w}^{2}\left(\eta_{1}, \eta_{2}\right)=\int\left|s_{\eta_{1}}(x \mid w)-s_{\eta_{2}}(x \mid w)\right|^{2} \psi_{\eta_{0}}(x \mid w) d \mu(x),
$$

is bounded by a constant multiple of $\int Q^{3}(x, w) d \Psi_{\eta_{0}}^{w}(x)$. Note that,

$$
h^{2}\left(\psi_{\eta_{1}}(\cdot \mid w), \psi_{\eta_{2}}(\cdot \mid w)\right)=2\left(1-\int \sqrt{\psi_{\eta_{1}}(x \mid w) \psi_{\eta_{2}}(x \mid w)} d \mu(x)\right) .
$$

Since,

$$
\frac{\partial \psi_{\eta}}{\partial w_{j}}(x \mid w)=\int\left(\dot{f}\left(x_{j}-w_{j}^{T} b\right) \prod_{k \neq j} f\left(x_{k}-w_{k}^{T} b\right)\right) \cdot b d G(b),
$$

where $\dot{f}$ is the derivative of $f$, we have,

$$
\left|\frac{\partial \psi_{\eta}(x \mid w) / \partial w_{j}}{\psi_{\eta}(x \mid w)}\right| \leq C \sup _{b}\left|\frac{\dot{f}\left(x_{j}-w_{j}^{T} b\right)}{f\left(x_{j}-w_{j}^{T} b\right)}\right|
$$


for a constant $C>0$, so the supremum of the left-hand side of $(\mathrm{A} .39)$ is of order $O\left(\left|x_{j}\right|\right)$, as $\left|x_{j}\right| \rightarrow \infty$, where the supremum is taken over $\eta \in \mathcal{H}_{0}$. Consequently,

$$
\begin{aligned}
\left|\frac{\partial}{\partial w_{j}} h^{2}\left(\psi_{\eta_{1}}(\cdot \mid w), \psi_{\eta_{2}}(\cdot \mid w)\right)\right| & \leq\left|\int \frac{\partial\left(\psi_{\eta_{1}}(x \mid w) \psi_{\eta_{2}}(x \mid w)\right) / \partial w_{j}}{\sqrt{\psi_{\eta_{1}}(x \mid w) \psi_{\eta_{2}}(x \mid w)}} d \mu(x)\right| \\
& \leq \int O(|x|) \times\left(\psi_{\eta_{1}}(x \mid w)+\psi_{\eta_{2}}(x \mid w)\right) d \mu(x) .
\end{aligned}
$$

Since $\sup _{w} \sup _{\eta \in \mathcal{H}_{0}} \int|x| d \Psi_{\eta}^{w}(x)<\infty$, this establishes the equicontinuity of 4.5).

\section{A.2.7 Proof of asymtoptic tightness of 4.6 in Corollary 4.1}

It only remains to prove asymptotic tightness of (4.6). Without loss of generality, we may assume that $\theta_{0}=0$. Let $\mathscr{F}=B_{\epsilon} \times \mathcal{H}_{0}$, where $B_{\epsilon}$ is the Euclidean ball of radius $\epsilon$ centered on $\theta_{0}$, and define $Z_{n i}(\theta, \eta), S_{n i}$, and $N_{[]}^{n}(\delta, \mathscr{F})$ as in the proof of Corollary 3.1. By the bracketing central limit theorem (Theorem 2.11.9 of [33]), it suffices to prove that,

$$
\begin{gathered}
\sum_{i=1}^{n} P_{0}\left(S_{n i} 1_{\left\{S_{n i}>\gamma\right\}}\right)=o(1), \quad \text { for every } \gamma>0, \\
\int_{0}^{\delta_{n}} \sqrt{\log N_{[]}^{n}(\delta, \mathscr{F})} d \delta<\infty, \quad \text { for every } \delta_{n} \downarrow 0 .
\end{gathered}
$$

The first condition of A.40 is proved in a manner similar to the proof of Corollary 3.1, by replacing $Q_{1}$ by $Q$ defined in 4.16.

To prove the second condition of A.40, note that,

$$
\begin{aligned}
\mid Z_{n i}\left(\theta_{1}, \eta_{1}\right)- & Z_{n i}\left(\theta_{2}, \eta_{2}\right) \mid \\
& \leq\left|Z_{n i}\left(\theta_{1}, \eta_{1}\right)-Z_{n i}\left(\theta_{2}, \eta_{1}\right)\right|+\left|Z_{n i}\left(\theta_{2}, \eta_{1}\right)-Z_{n i}\left(\theta_{2}, \eta_{2}\right)\right| .
\end{aligned}
$$

By (4.16), $\sup _{i \geq 1} \sup _{\eta \in \mathcal{H}_{0}}\left|\dot{\ell}_{\theta, \eta, i}-\dot{\ell}_{\theta_{0}, \eta, i}\right|$ is bounded by a constant multiple of $\left|\theta_{1}-\theta_{2}\right|$, and so there exists a constant $K_{1}>0$ such that,

$$
\sup _{\eta \in \mathcal{H}_{0}}\left|Z_{n i}\left(\theta_{1}, \eta\right)-Z_{n i}\left(\theta_{2}, \eta\right)\right| \leq \frac{K_{1}}{\sqrt{n}}\left|\theta_{1}-\theta_{2}\right|
$$

Let $g_{\eta, j}(x \mid w)=\partial \ell_{\eta}(x \mid w) / \partial x_{j}$, and for $t>0$, let,

$$
\mathcal{S}_{t}=\left\{(x, w) \mapsto g_{\eta, j}(x \mid w): \eta \in \mathcal{H}_{0}, 1 \leq j \leq m\right\},
$$

where functions in $\mathcal{S}_{t}$ are viewed as maps from $[-t, t]^{m} \times[-L, L]^{q m}$ to $\mathbb{R}$. Since $w$ ranges over a compact set and $G$ is supported on a compact set, the $\alpha$-th order partial derivative of the map $(x, w) \mapsto \psi_{\eta}(x \mid w)$ is bounded by a constant multiple of $|x|^{\alpha} \psi_{\eta}(x \mid w)$ for every $\eta \in \mathcal{H}_{0}, w$, and large enough $|x|$. Thus, for some constant $D_{\alpha}>0$, the $\alpha$-Hölder norm of functions in $\mathcal{S}_{t}$ is bounded by $D_{\alpha} t^{\alpha+1}$ for large enough $t$. Since the Lebesgue measure of $[-t, t]^{m} \times[-L, L]^{q m}$ is bounded by a constant multiple of $t^{m}$, applying Theorem 2.7.1 of [33] with $\alpha=d=(q+1) m$, there exists a constant $K_{2}>0$ such that,

$$
\log N\left(\delta, \mathcal{S}_{t},\|\cdot\|_{\infty}\right)<K_{2} \frac{t^{(q+2) m+1}}{\delta}
$$


for every $\delta>0$ and large enough $t>0$. Since $\sup _{w} \sup _{\eta \in \mathcal{H}_{0}}\left|s_{\eta}(x \mid w)\right|=O(|x|)$ and $\sup _{w} \psi_{\eta_{0}}(x \mid w)=O\left(\exp \left(-K_{3}|x|^{2}\right)\right)$ as $|x| \rightarrow \infty$ for some constant $K_{3}>0$, we have,

$$
\int_{\left\{|x|>M_{\delta}\right\}} \sup _{|y| \leq \sqrt{m} L \epsilon} \sup _{w} \sup _{\eta \in \mathcal{H}_{0}}\left|s_{\eta}(x+y \mid w)\right|^{2} \psi_{\eta_{0}}(x \mid w) d \mu(x) \leq \delta^{2},
$$

for every small enough $\delta>0$, where $M_{\delta}=-\log \delta$. Therefore, for every small enough $\delta>0$ we can construct a partition $\mathcal{H}_{0}=\cup_{l=1}^{N_{\delta}} \mathcal{H}^{(l)}$ such that, for some constant $K_{4}>0$,

$$
\log N_{\delta} \leq K_{4}\left|\log \frac{1}{\delta}\right|^{(q+2) m+1} \frac{1}{\delta}
$$

and,

$$
\int \sup _{\theta \in B_{\epsilon}} \sup _{i \geq 1} \sup _{\eta_{1}, \eta_{2} \in \mathcal{H}^{(l)}}\left|Z_{n i}\left(\theta, \eta_{1}\right)-Z_{n i}\left(\theta, \eta_{2}\right)\right|^{2} \psi_{\eta_{0}}\left(x \mid W_{i}\right) d \mu(x)<\frac{\delta^{2}}{n},
$$

for every $l \leq N_{\delta}$. Since $N\left(\delta, B_{\epsilon},|\cdot|\right)=O\left(\delta^{-p}\right)$ as $\delta \rightarrow 0$, A.41, A.42 and A.43 imply that,

$$
\log N_{[]}^{n}(\delta, \mathscr{F}) \leq K_{5}\left(\left|\log \frac{1}{\delta}\right|^{(q+2) m+1} \frac{1}{\delta}+\left|\log \frac{1}{\delta}\right|\right) \leq\left(\frac{1}{\delta}\right)^{3 / 2},
$$

for some $K_{5}>0$, so the second condition of A.40 is satisfied.

\section{A.2.8 Proof of asymptotic tightness of 4.6 in Corollary 4.2}

Without loss of generality, we may assume that $\theta_{0}=0$. Let $\mathscr{F}=B_{\epsilon} \times \mathcal{H}_{0}$, where $B_{\epsilon}$ is the Euclidean ball of radius $\epsilon$ centered on $\theta_{0}$, and define $Z_{n i}(\theta, \eta), S_{n i}$, and $N_{[]}^{n}(\delta, \mathscr{F})$ as those defined in the proof of Corollary 4.1. By the bracketing central limit theorem (Theorem 2.11.9 of [33]), it is sufficient to prove A.40. The first condition of A.40 is easily satisfied. For the second condition of (A.40), the inequalities (A.41) and (A.42) hold similarly. Thus for every $\delta>0$, it suffices to construct a partition $\mathcal{H}_{0}=\cup_{l=1}^{N_{\delta}} \mathcal{H}^{(l)}$ satisfying $\mathrm{A} .43$ and $\log N_{\delta} \leq \delta^{-2+\beta}$ for some $\beta>0$.

For $f \in \mathcal{F}_{0}$, let $\dot{\ell}_{f}$ be the derivatives of $\log f$, and let,

$$
\mathcal{L}=\mathcal{F}_{0} \cup\left\{\dot{\ell}_{f}: f \in \mathcal{F}_{0}\right\}
$$

Since functions in $\mathcal{L}$ and their derivatives are uniformly bounded, applying Theorem 2.7.1 of [33] with $\alpha=d=1$, there exists a constant $K_{1}>0$ such that,

$$
\log N\left(\delta, \mathcal{L},\|\cdot\|_{\infty}\right) \leq K_{1} \frac{1}{\delta},
$$

for every $\delta>0$. Since $\mathcal{G}_{0}$ is parametrized by a covariance matrix $\Sigma$, it is compact in the induced matrix norm $\|\cdot\|$, and,

$$
\log N\left(\delta, \mathcal{G}_{0},\|\cdot\|\right) \leq K_{2} \log \left(\frac{1}{\delta}\right),
$$


for some $K_{2}>0$. Note that in $\mathcal{G}_{0}, d_{V}$ is bounded by a constant multiple of $\|\cdot\|$ because the density of the normal distribution $N(0, \Sigma)$ is differentiable and its derivative is uniformly bounded, because $\rho_{\min }(\Sigma)>\rho_{1}$. Since $d_{W} \leq d_{V}$ (see [15]), we have that,

$$
\log N\left(\delta, \mathcal{G}_{0}, d_{W}\right) \leq K_{3} \log \left(\frac{1}{\delta}\right)
$$

for some $K_{3}>0$. Note that,

$$
\frac{\partial \ell_{\eta}}{\partial x_{k}}(x \mid w)=\frac{\int \dot{\ell}_{f}\left(x_{k}-b^{T} w_{k}\right) \prod_{j=1}^{m} f\left(x_{j}-b^{T} w_{j}\right) d G(b)}{\int \prod_{j=1}^{m} f\left(x_{j}-b^{T} w_{j}\right) d G(b)} .
$$

Since the denominator of the last display is bounded away from zero and $b / a-d / c=$ $b(c-a) / a c+(b-d) / c$ for every real numbers $a, b, c, d$ with $a c \neq 0$, there exists a constant $K_{4}>0$ such that for every $f_{1}, f_{2} \in \mathcal{F}_{0}$,

$$
\sup _{G \in \mathcal{G} x, w}\left|\frac{\partial \ell_{\left(f_{1}, G\right)}}{\partial x_{k}}(x \mid w)-\frac{\partial \ell_{\left(f_{1}, G\right)}}{\partial x_{k}}(x \mid w)\right| \leq K_{4}\left(\left\|f_{1}-f_{2}\right\|_{\infty} \vee\left\|\dot{\ell}_{f_{1}}-\dot{\ell}_{f_{2}}\right\|_{\infty}\right) .
$$

Also, by Theorem 2 of [15], there exists a constant $K_{5}>0$ such that for every $G_{1}, G_{2} \in \mathcal{G}$,

$$
\sup _{f \in \mathcal{F}_{0}} \sup _{x, w}\left|\frac{\partial \ell_{\left(f, G_{1}\right)}}{\partial x_{k}}(x \mid w)-\frac{\partial \ell_{\left(f, G_{2}\right)}}{\partial x_{k}}(x \mid w)\right| \leq K_{5} d_{W}\left(G_{1}, G_{2}\right) .
$$

Therefore, by (A.44) and A.45, A.43 is satisfied with entropy bound,

$$
\log N_{\delta} \leq K_{6}\left(\frac{1}{\delta}+\log \left(\frac{1}{\delta}\right)\right)
$$

for some $K_{6}>0$.

\section{Acknowledgements}

The first author thanks to thesis committee members for valuable suggestions. BK also thanks the Statistics Department of Seoul National University, South Korea for its kind hospitality.

\section{References}

[1] Barron, A., Schervish, M. J., and Wasserman, L. (1999). The consistency of posterior distributions in nonparametric problems. The Annals of Statistics, 27(2):536-561.

[2] Beran, R. (1978). An efficient and robust adaptive estimator of location. The Annals of Statistics, 6(2):292-313.

[3] Bickel, P. and Kleijn, B. (2012). The semiparametric Bernstein-von Mises theorem. The Annals of Statistics, 40(1):206-237.

[4] Bickel, P. J. (1982). On adaptive estimation. The Annals of Statistics, 10(3):647-671. 
[5] Bontemps, D. (2011). Bernstein-von Mises theorems for Gaussian regression with increasing number of regressors. The Annals of Statistics, 39(5):2557-2584.

[6] Boucheron, S. and Gassiat, E. (2009). A Bernstein-von Mises theorem for discrete probability distributions. Electronic Journal of Statistics, 3:114-148.

[7] Castillo, I. and Rousseau, J. (2015). A Bernstein-von Mises theorem for smooth functionals in semiparametric models. The Annals of Statistics, 43(6):2353-2383.

[8] Chae, M. (2015). The semiparametric Bernstein-von Mises theorem for models with symmetric error. PhD thesis, Seoul National University. arXiv:1510.0524\%.

[9] Cox, D. D. (1993). An analysis of Bayesian inference for nonparametric regression. The Annals of Statistics, 21(2):903-923.

[10] Ferguson, T. S. (1973). A Bayesian analysis of some nonparametric problems. The Annals of Statistics, 1(2):209-230.

[11] Freedman, D. (1999). Wald Lecture: On the Bernstein-von Mises theorem with infinite-dimensional parameters. The Annals of Statistics, 27(4):1119-1141.

[12] Ghosal, S. (1999). Asymptotic normality of posterior distributions in highdimensional linear models. Bernoulli, 5(2):315-331.

[13] Ghosal, S. and van der Vaart, A. W. (2001). Entropies and rates of convergence for maximum likelihood and Bayes estimation for mixtures of normal densities. The Annals of Statistics, 29(5):1233-1263.

[14] Ghosal, S. and van der Vaart, A. W. (2007). Convergence rates of posterior distributions for noniid observations. The Annals of Statistics, 35(1):192-223.

[15] Gibbs, A. L. and Su, F. E. (2002). On choosing and bounding probability metrics. International statistical review, 70(3):419-435.

[16] Henderson, C. R. (1975). Best linear unbiased estimation and prediction under a selection model. Biometrics, pages 423-447.

[17] Johnstone, I. M. (2010). High dimensional Bernstein-von Mises: simple examples. Institute of Mathematical Statistics Collections, 6:87-98.

[18] Kim, Y. (2006). The Bernstein-von Mises theorem for the proportional hazard model. The Annals of Statistics, 34(4):1678-1700.

[19] Kim, Y. and Lee, J. (2004). A Bernstein-von Mises theorem in the nonparametric right-censoring model. The Annals of Statistics, 32(4):1492-1512.

[20] Kleijn, B. (2013). Criteria for Bayesian consistency. arXiv:1308.1263. 
[21] Kleijn, B. and van der Vaart, A. (2012). The Bernstein-von Mises theorem under misspecification. Electronic Journal of Statistics, 6:354-381.

[22] Le Cam, L. (1973). Convergence of estimates under dimensionality restrictions. The Annals of Statistics, 1(1):38-53.

[23] Le Cam, L. and Yang, G. L. (1990). Asymptotics in statistics: some basic concepts. Springer, New York.

[24] Lo, A. Y. (1984). On a class of Bayesian nonparametric estimates: I. Density estimates. The Annals of Statistics, 12(1):351-357.

[25] McNeney, B. and Wellner, J. A. (2000). Application of convolution theorems in semiparametric models with non-iid data. Journal of Statistical Planning and Inference, 91(2):441-480.

[26] Panov, M. and Spokoiny, V. (2015). Finite sample Bernstein-von Mises theorem for semiparametric problems. Bayesian Analysis, 10(3):665-710.

[27] Sacks, J. (1975). An asymptotically efficient sequence of estimators of a location parameter. The Annals of Statistics, 3(2):285-298.

[28] Schwartz, L. (1965). On Bayes procedures. Zeitschrift für Wahrscheinlichkeitstheorie und verwandte Gebiete, 4(1):10-26.

[29] Shen, X. (2002). Asymptotic normality of semiparametric and nonparametric posterior distributions. Journal of the American Statistical Association, 97(457):222-235.

[30] Spokoiny, V. (2013). Bernstein-von Mises theorem for growing parameter dimension. arXiv:1302.3430.

[31] Stone, C. J. (1975). Adaptive maximum likelihood estimators of a location parameter. The Annals of Statistics, 3(2):267-284.

[32] Taylor, A. E. (2012). General theory of functions and integration. Courier Corporation.

[33] van der Vaart, A. and Wellner, J. A. (1996). Weak convergence and empirical processes. Springer Verlag.

[34] van der Vaart, A. W. (1996). Efficient maximum likelihood estimation in semiparametric mixture models. The Annals of Statistics, 24(2):862-878.

[35] van der Vaart, A. W. (1998). Asymptotic statistics. Cambridge series in statistical and probabilistic mathematics, volume 3 . Cambridge university press. 
[36] Walker, S. (2004). New approaches to Bayesian consistency. The Annals of Statistics, $32(5): 2028-2043$.

[37] Wong, W. H. and Shen, X. (1995). Probability inequalities for likelihood ratios and convergence rates of sieve MLEs. The Annals of Statistics, 23(2):339-362.

[38] Yang, Y., Cheng, G., and Dunson, D. B. (2015). Semiparametric Bernstein-von Mises theorem: Second order studies. arXiv:1503.04493. 